Conservação de momento angular em sistemas mecânicos dissipativos

Lucas Ruiz dos Santos

\author{
DISSERTAÇÃO APRESENTADA \\ $\mathrm{AO}$ \\ Instituto De Matemática e Estatística \\ DA \\ Universidade DE SÃo PaUlo \\ PARA \\ OBTENÇÃO DO TÍTULO \\ DE \\ MESTRE EM CIÊNCIAS
}

Programa: Pós graduação em Matemática Aplicada

Orientador: Prof. Dr. Clodoaldo Grotta Ragazzo

Durante o desenvolvimento deste trabalho o autor recebeu auxílio financeiro do CNPq

São Paulo, 16 de fevereiro de 2012 


\section{Conservação de momento angular em sistemas mecânicos dissipativos}

Esta dissertação contém as correções e alterações sugeridas pela Comissão Julgadora durante a defesa realizada por Lucas Ruiz dos Santos em 14/02/2012.

O original encontra-se disponível no Instituto de Matemática e Estatística da Universidade de São Paulo.

Comissão Julgadora:

- Prof. Dr. Clodoaldo Grotta Ragazzo (orientador) - IME-USP

- Profa. Dra. Tatiana Alexandrovna Michtchenko - IAG-USP

- Prof. Dr. Gláucio Terra - IME-USP 


\section{Resumo}

RUIZ, L. S. Conservação de momento angular em sistemas mecânicos dissipativos. 2012. 59f. Dissertação de mestrado - Instituto de Matemática e Estatística, Universidade de São Paulo, São Paulo, 2012.

Objetiva-se discutir e explorar as consequências da conservação do momento angular em situações físicas que apresentem dissipação de energia. Frequentemente, sistemas mecânicos dissipativos são omitidos nas formulações matemáticas da mecânica clássica. No entanto, a conservação da energia mecânica é uma idealização drasticamente incompatível, a longos períodos de tempo, com a maioria das situações reais. Apesar de parecer controversa, esta abordagem é compreensível desde que é incluída na dissipação toda a complexidade dos sistemas mecânicos, os quais não podemos modelar completamente. Espera-se assim que os resultados obtidos sejam parcialmente comprovados em experimentos, sendo que a discrepância é atribuída à parte desconhecida do fenômeno, que muitas vezes é considerada irrelevante para fins práticos. Devido à mencionada complexidade de fenômenos dissipativos, a postura adotada nesta dissertação foi analisar específicos exemplos, de natureza distinta, simplificados, que apresentem as duas propriedades em questão. Concentrou-se especialmente no problema de dissipação de energia em corpos deformáveis na ausência de estímulos externos.

Palavras-chave: momento angular, dissipação de energia, corpo rígido. 


\section{Abstract}

\section{RUIZ, L. S. Conservation of angular momentum in dissipative mechanical systems.}

2012. 59f. Dissertação de mestrado - Instituto de Matemática e Estatística, Universidade de São Paulo, São Paulo, 2012.

The goal of this work is to study physical systems where angular momentum is a conserved quantity while energy is dissipated. It is a common practice among people working on classical mechanics to neglect energy dissipation. While this hypothesis leads to beautiful mathematical theories, it is strongly violated by the majority of real world systems. The physical complexity of the many ways in which energy is dissipated turns the mathematical modelling of dissipative forces troublesome. There is essentially no general mathematical model for dissipative forces in good agreement with a wide range of experiments. This is in contrast to the very few accepted conservative force models: gravity, elasticity and magneto-electricity. The dissipative force model usually varies among the various physical situations. So, in this work several systems where energy is dissipated while angular momentum is conserved will be considered. The main example will be the motion of a deformable body free from external forces and torques.

Keywords: angular momentum, energy dissipation, rigid body. 


\section{Sumário}

Lista de Figuras $\quad$ vii

1 Introdução 1

2 Motivação $\quad 5$

2.1 Modelo pontual de dissipação . . . . . . . . . . . . . . . . . . . . . 5

2.2 Dissipação interna em um corpo isolado . . . . . . . . . . . . . . . . . . 9

2.3 Oscilador harmônico amortecido acoplado a corpo rígido livre . . . . . . . . . . . . . 14

3 Equações de movimento $\quad 21$

3.1 Conceitos preliminares . . . . . . . . . . . . . . . . . . 21

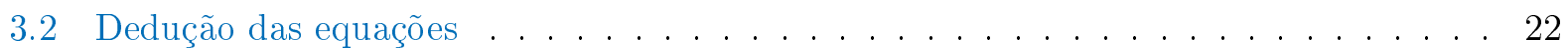

4 Modelos $\quad 39$

4.1 Hastes flexíveis fixas a corpos rígidos . . . . . . . . . . . . . . . . . . 39

4.2 Caso de rotação restrita a um plano . . . . . . . . . . . . . . . . . 43

4.3 Aplicação ao corpo rígido com oscilador . . . . . . . . . . . . . . . . . . 46

4.4 Elasticidade tridimensional . . . . . . . . . . . . . . . . . 47

4.5 Tempo de relaxação . . . . . . . . . . . . . . . . . . . . . . . . . 49

Conclusões $\quad \mathbf{5 5}$

4.5 .1 Propostas de estudo . . . . . . . . . . . . . . . 57

A Princípios de Galileu $\quad 59$

$\begin{array}{ll}\text { B Equações de movimento para problema restrito } & 61\end{array}$

$\begin{array}{ll}\text { Referências Bibliográficas } & 63\end{array}$ 


\section{Lista de Figuras}

2.1 Níveis negativos da energia mecânica. . . . . . . . . . . . . . . . 7

2.2 Comportamento qualitativo das soluções. . . . . . . . . . . . . . . . 7

2.3 Regiões de energia negativa para momento angular nulo. . . . . . . . . . . . 8

2.4 Retrato de fase para a velocidade angular no referencial do corpo rígido. . . . . . . . . . 12

2.5 Retrato de fase para a velocidade angular no referencial do corpo com dissipação. . . . . . . 13

2.6 Corpo rígido acoplado a oscilador harmônico amortecido. . . . . . . . . . . . . . . . 14

4.1 Corpo rígido livre com uma haste agregada. . . . . . . . . . . . . . . . . 40

4.2 Haste elástica anexa a disco rígido submetido a movimento plano . . . . . . . . . . . . 44 


\section{Capítulo 1}

\section{Introdução}

Comecemos com a equação fundamental da mecânica Newtoniana de partículas:

$$
m_{i} \ddot{x}_{i}=f_{i}\left(t, x_{1}, \ldots, x_{N}, \dot{x}_{1}, \ldots, \dot{x}_{N}\right), \quad i=1, \ldots N, \quad t \in \mathbb{R}, \quad x_{i} \in \mathbb{R}^{3} .
$$

Esta equação descreve o movimento de um sistema de $N$ partículas no espaço, com massas $m_{i}$ e posições $x_{i}$, interagindo entre si e com o meio. Desenvolvida de maneira quase simultânea ao cálculo, proporcionou à ciência um assombroso avanço.

Apesar de terem sido evidenciados os limites de seu domínio de validade, são inegáveis sua eficiência e vasta generalidade de aplicações. Nesta forma apresentada, pode descrever movimento de sistema de partículas isolado ou interagindo com um agente externo, podendo as partículas estar restritas a uma superfície por exemplo. Pode-se partir destas equações para desenvolver teorias de movimento de corpos contínuos sólidos, ou fluidos.

No entanto, não se afirma que as bases da mecânica Newtoniana se reduzem a esta equação.

Durante a modelagem de um problema, é necessário imaginar, observar ou realizar medidas sobre uma dada situação física, de forma a ganhar afinidade, formular hipóteses e concluir qual é uma boa expressão para as funções $f_{i}$, força sobre a $i$-ésima partícula. Para um sistema mecânico "isolado" (situação idealizada), postula-se que valem os princípios de Galileu, os quais são apresentados no apêndice A. Entre suas consequências, estão que as forças não dependem explicitamente do tempo, e também que dependem somente das posições e velocidades relativas entre as partículas. Ou seja, a dependência temporal nas forças é consequência de um agente externo. Nesta situação, uma combinação do princípio de superposição, da terceira lei de Newton e da isotropia do espaço implica que:

$$
f_{i}\left(x_{1}, \ldots, x_{N}, \dot{x}_{1}, \ldots, \dot{x}_{N}\right)=\sum_{j=1}^{N} \phi_{i j}\left(x_{j}-x_{i}, \dot{x}_{j}-\dot{x}_{i}\right)\left(x_{j}-x_{i}\right) .
$$

Onde $\phi_{i j}\left(x_{j}-x_{i}, \dot{x}_{j}-\dot{x}_{i}\right)=\phi_{j i}\left(x_{i}-x_{j}, \dot{x}_{i}-\dot{x}_{j}\right), \forall i, j$.

Estas hipóteses bastam para que seja garantida a conservação do momento angular total do sistema, qual seja:

$$
\begin{gathered}
M: \mathbb{R}^{6 N} \longrightarrow \mathbb{R}^{3} \\
M\left(x_{1}, \ldots, x_{N}, \dot{x}_{1}, \ldots, \dot{x}_{N}\right):=\sum_{i=1}^{N} m_{i} x_{i} \times \dot{x}_{i} .
\end{gathered}
$$

De fato, se $\left(x_{1}, \ldots, x_{N}\right)$ é solução,

$$
\frac{d}{d t} M\left(x_{1}, \ldots, x_{N}, \dot{x}_{1}, \ldots, \dot{x}_{N}\right)=\sum_{i=1}^{N} x_{i} \times\left(m_{i} \ddot{x}_{i}\right)=\sum_{i, j=1}^{N} \phi_{i j}\left(x_{j}-x_{i}, \dot{x}_{j}-\dot{x}_{i}\right) x_{i} \times\left(x_{j}-x_{i}\right)=
$$




$$
\begin{gathered}
=\sum_{i, j=1}^{N} \phi_{i j}\left(x_{j}-x_{i}, \dot{x}_{j}-\dot{x}_{i}\right) x_{i} \times x_{j}=\sum_{i<j}^{N} \phi_{i j}\left(x_{j}-x_{i}, \dot{x}_{j}-\dot{x}_{i}\right) x_{i} \times x_{j}+\sum_{i>j}^{N} \phi_{i j}\left(x_{j}-x_{i}, \dot{x}_{j}-\dot{x}_{i}\right) x_{i} \times x_{j}= \\
=\sum_{i<j}^{N}\left(\phi_{i j}\left(x_{j}-x_{i}, \dot{x}_{j}-\dot{x}_{i}\right)-\phi_{j i}\left(x_{i}-x_{j}, \dot{x}_{i}-\dot{x}_{j}\right)\right) x_{i} \times x_{j}=0 .
\end{gathered}
$$

Restringindo ainda mais o problema, podemos formular a hipótese (não intuitiva) de que exista uma função $V: \mathbb{R}^{6 N} \longrightarrow \mathbb{R}^{3}$ tal que $f_{i}=-\operatorname{grad}_{x_{i}} V\left(x_{1}, \ldots, x_{N}, \dot{x}_{1}, \ldots, \dot{x}_{N}\right)$. Onde $\operatorname{grad}_{x_{i}}$ é o gradiente com respeito às coordenadas da $i$-ésima partícula.

Neste caso, define-se a energia mecânica total do sistema:

$$
E:=\sum_{i=1}^{N} T_{i}+V .
$$

Onde $T_{i}:=m_{i} \dot{x}_{i}^{2} / 2$, é a energia cinética da $i$-ésima partícula.

Assim, ao longo das soluções da equação de Newton, temos:

$$
\begin{gathered}
\dot{E}=\sum_{i=1}^{N}-<\dot{x}_{i}, \operatorname{grad}_{x_{i}} V>+\sum_{i=1}^{N}<\operatorname{grad}_{x_{i}} V, \dot{x}_{i}>+<\operatorname{grad}_{\dot{x}_{i}} V, \ddot{x}_{i}>= \\
=-\sum_{i=1}^{N} \frac{1}{m_{i}}<\operatorname{grad}_{\dot{x}_{i}} V, \operatorname{grad}_{x_{i}} V>
\end{gathered}
$$

Sob as hipóteses acima, esta expressão, em geral, será não nula.

No entanto, supondo adicionalmente que $V$ não dependa das velocidades (e consequentemente cada $f_{i}$ ), obtemos a conservação da energia.

Note que as hipóteses acima são suficientes para as conservações de energia e momento angular, mas não foi assegurado que são necessárias.

As deduções acima levam a se esperar que a conservação de momento angular, que requer menos hipóteses sob a força, seja mais frequente que a conservação de energia.

Considere o exemplo do sistema Terra-Lua (ignorando a interação com os demais corpos celestes). Em primeira aproximação, o problema pode ser tratado (segundo a mecânica Newtoniana) como duas partículas interagindo gravitacionalmente. Em um segundo momento, pode-se considerar a atuação da força gravitacional sobre a crosta terrestre e o oceano. Esta interação induz um movimento que gera calor. Este pode ser pensado como convertido a partir da energia cinética ou da interação gravitacional Terra-Lua. Esta "dissipação de energia" pode alterar consideravelmente o movimento descrito pelo modelo ideal? Este tipo de pergunta é uma das motivações para a escolha do tema.

Considere agora o sistema que é um oscilador harmônico amortecido. Sem atrito, todas as soluções são periódicas. Com a adição de um termo dissipativo, todas as soluções tendem ao equilíbrio, com a mola relaxada e com a massa em repouso. Este é o papel que usualmente se atribui à dissipação de energia: para tempos longos, faz o sistema entrar completamente em repouso, evidenciando que o modelo ideal é apenas uma aproximação para pequenos intervalos de tempo.

No entanto, suponha que dada uma condição inicial, o momento angular seja conservado e não nulo. Assumindo a presença de atrito, ou outro mecanismo de dissipação, esperamos que a velocidade de todas as partículas se aproxime de zero a longo tempo. Supondo que elas não podem se afastar arbitrariamente, o momento angular não poderia se manter constante. Espera-se então que o movimento das partículas não se "trivialize" com o tempo, o que desperta maior interesse pelo assunto.

As equações de Newton para sistemas mecânicos conservativos possuem diversas formulações distintas, como por exemplo a Lagrangiana e a Hamiltoniana. Para sistemas mecânicos dissipativos isso não ocorre. Algumas classes de forças dissipativas podem ser descritas no formalismo 
Lagrangiano através da introdução da chamada "função de dissipação de Rayleigh", $\mathcal{D}$. Neste formalismo, sendo $L=T-V$ a função Lagrangiana, as equações de movimento ficam:

$$
\frac{d}{d t}\left(\frac{\partial L}{\partial \dot{q}}\right)-\frac{\partial L}{\partial q}=-\frac{\partial \mathcal{D}}{\partial \dot{q}}
$$

Por exemplo, para o oscilador amortecido, $T=m \dot{x}^{2} / 2, V=k x^{2} / 2$ e $\mathcal{D}=c \dot{x}^{2} / 2$. Não foi encontrado algum princípio variacional que resultasse nestas equações, como acontece para o caso conservativo. No entanto, elas possuem uma propriedade de invariância por mudanças de coordenadas, como as usuais equações de Euler-Lagrange. Mais precisamente, suponha que o sistema esteja definido em um aberto de $\mathbb{R}^{N}$ (sem vínculos). Suponha que $Q(t)$ seja solução desta equação. Se $Q \rightarrow q(Q)$ é uma mudança de coordenadas defina, $L_{1}(Q, \dot{Q}):=L\left(q(Q), q^{\prime}(Q) \dot{Q}\right), \mathcal{D}_{1}(Q, \dot{Q}):=\mathcal{D}\left(q(Q), q^{\prime}(Q) \dot{Q}\right)$. Assim,

$$
\begin{gathered}
\partial_{Q} L_{1}=\partial_{q} L \circ q^{\prime}(Q)+\partial_{\dot{q}} L \circ d^{2} q(Q)(\dot{Q}, \cdot), \quad \partial_{\dot{Q}} L_{1}=\partial_{\dot{q}} L \circ q^{\prime}(Q), \quad \partial_{\dot{Q}} \mathcal{D}_{1}=\partial_{\dot{q}} \mathcal{D} \circ q^{\prime}(Q) \\
\Rightarrow \frac{d}{d t} \partial_{\dot{Q}} L_{1}=\frac{d}{d t}\left(\partial_{\dot{q}} L\right) \circ q^{\prime}(Q)+\partial_{\dot{q}} L \circ d^{2} q(Q)(\dot{Q}, \cdot) .
\end{gathered}
$$

Onde $d^{2} q(Q)$ é o Hessiano de $q(Q)$. Assim, usando o produto interno canônico,

$$
\frac{d}{d t}\left(\frac{\partial L_{1}}{\partial \dot{Q}}\right)-\frac{\partial L_{1}}{\partial Q}+\frac{\partial \mathcal{D}_{1}}{\partial \dot{Q}}=q^{\prime}(Q)^{T}\left[\frac{d}{d t}\left(\frac{\partial L}{\partial \dot{q}}\right)-\frac{\partial L}{\partial q}+\frac{\partial \mathcal{D}}{\partial \dot{q}}\right]
$$

Como $q(Q)$ é difeo, o primeiro termo se anula se e só se o segundo também é nulo. Observe que foi suposto que $L$ não depende explicitamente do tempo.

A mecânica Newtoniana para sistemas de partículas pode ser estendida para sistemas contínuos, a chamada mecânica do contínuo. As dificuldades matemáticas enfrentadas na mecânica do contínuo são muito maiores do que aquelas dos sistemas de partículas finitos. Mesmo assim, sob certas hipóteses, muitos dos resultados válidos para sistemas de partículas são generalizados para sistemas contínuos.

Em particular, ocorre a situação onde o movimento de um corpo contínuo apresenta conservação de momento angular e dissipação de energia. Trata-se de um sólido em rotação não submetido a forças externas. Este problema será analisado com detalhes ao longo do texto. Para tal, será utilizado um formalismo Lagrangiano, por analogia com o já citado. Por uma série de argumentos heurísticos, como será feito na seção 2.2, afirma-se que qualquer configuração inicial deste sistema evolui para uma rotação estacionária em torno do eixo de maior inércia (da configuração de equilíbrio). Aqui, novamente, a conservação do momento angular é a evidência clara de que o corpo não pode cessar sua rotação.

Este modelo possui diversas aplicações. Suas soluções são interessantes na observação da precessão de asteróides e cometas, e o chamado "alinhamento de poeira cósmica". Para referências detalhadas sobre estes fenômenos, o leitor pode consultar [EL00], [Efr00], [Efr01], [ELS02], [LE99] e [SBH05]. O comportamento assintótico do modelo também é de interesse no projeto de satélites artificiais. Em geral, estes são colocados em órbita em torno da Terra de forma estacionária com respeito ao solo. Esta é uma situação na qual pode ser usada a hipótese de ausência de forças externas, desde que no referencial do corpo a atração gravitacional seja "compensada pelas forças inerciais". A questão do movimento rotacional de satélites ganhou evidência após o inesperado comportamento do satélite Explorer I, o primeiro satélite americano a ser colocado em órbita com sucesso, em 1958. O satélite, que possuía um formato cilíndrico alongado, foi projetado para girar em torno do eixo de menor inércia. No entanto, antes de dar uma volta completa em torno da Terra, o mesmo passou a precessar de forma irregular, com amplitude cada vez maior. Esta questão foi tratada por diversos autores. Aqui será seguido aquele de M. Levi e J. Baillieul nas referências [BL87] e [BL91].

Esta dissertação está dividida da seguinte forma:

- O capítulo 2 é dedicado à análise de sistemas que motivaram o trabalho, quais sejam, o 
problema de Kepler e a dinâmica de um sólido livre, ambos com dissipação.

- No capítulo 3, este último sistema é formulado de maneira geral e são obtidos alguns resultados.

- No capítulo 4, são dadas aplicações da formulação para casos específicos e é apresentada no final uma abordagem alternativa a um dos modelos.

- Na conclusão, são sugeridas questões que surgiram durante o estudo. 


\section{Capítulo 2}

\section{Motivação}

Este capítulo é dedicado à análise dos sistemas mecânicos cujas analogias motivaram o presente estudo. A dissipação de energia e conservação do momento angular estão presentes em todos eles.

\subsection{Modelo pontual de dissipação}

Examinemos a seguinte modelagem (bastante simplificada) do sistema isolado de dois corpos interagindo gravitacionalmente.

Serão desprezadas suas dimensões espaciais, tratando-os como partículas. A massa de um dos corpos será considerada muito maior que a do outro, adotando-se como referencial inercial o centro de massa deste. Ainda, a interação será "perturbada" pela presença de uma força dependente da velocidade relativa (uma força que pode ser simplificação da força de maré).

Assim como no caso conservativo (não perturbado), o movimento é restrito a um plano.

Denote por $x(t) \in \mathbb{R}^{2}$ a posição do menor corpo em relação ao maior, $r:=\|x\|$, e $V(r):=-k / r$. Onde $k$ é uma constante positiva.

Desta forma, $x(t)$ é solução do seguinte sistema de equações:

$$
\left\{\begin{aligned}
\dot{x} & =u \\
\dot{u} & =-V^{\prime}(r) \frac{x}{r}-\nu(x \cdot u) \frac{x}{r^{2}}
\end{aligned}\right.
$$

Onde $\nu$ é um parâmetro positivo.

Observe que este sistema pode ser obtido pelo formalismo Lagrangiano com função dissipação:

$$
\mathcal{D}(x, \dot{x}):=\frac{\nu}{2} \frac{(x \cdot \dot{x})^{2}}{r^{2}}
$$

Definindo, como usual, a energia mecânica do sistema: $\mathrm{E}(t):=\|u\|^{2} / 2+V(r)$, segue que:

$$
\dot{\mathrm{E}}=u \cdot \dot{u}+V^{\prime}(r) \frac{x}{r} \cdot \dot{x}=-V^{\prime}(r) \frac{x \cdot u}{r}-\nu \frac{(x \cdot u)^{2}}{r^{2}}+V^{\prime}(r) \frac{x}{r} \cdot \dot{x}=-\nu \frac{(x \cdot u)^{2}}{r^{2}}=-2 \mathcal{D} \leq 0 .
$$

Portanto, em geral, a energia mecânica não será uma integral primeira deste sistema. Sendo uma função decrescente do tempo.

No entanto, observando o momento angular, $L:=x_{1} u_{2}-x_{2} u_{1}$, constata-se diretamente que $\dot{L}=0$. Portanto, neste modelo modificado, $L$ continua sendo uma integral primeira.

Considere agora a mudança (local) de coordenadas dada implicitamente através das equações:

$$
\left\{\begin{array}{l}
x_{1}=r \cos \theta \\
x_{2}=r \operatorname{sen} \theta \\
u_{1}=-L \operatorname{sen} \theta / r+\eta \cos \theta \\
u_{2}=L \cos \theta / r+\eta \operatorname{sen} \theta
\end{array}\right.
$$


Nestas coordenadas, o sistema se escreve como:

$$
\left\{\begin{aligned}
\dot{L} & =0 \\
\dot{\theta} & =\frac{L}{r^{2}} \\
\dot{r} & =\eta \\
\dot{\eta} & =-\nu \eta-\frac{k}{r^{2}}+\frac{L^{2}}{r^{3}}
\end{aligned}\right.
$$

Sendo assim, considere o campo vetorial $F$ sobre (um aberto de) $\mathbb{R}^{2}$ dado pelas duas últimas equações do sistema acima: $F(r, \eta)=\left(\eta,-\nu \eta-k / r^{2}+L^{2} / r^{3}\right)$.

Suponha que o momento angular (constante) seja não nulo. Assim, $F$ somente se anula (para $r>0)$ em $\left(r_{0}, \eta_{0}\right)=\left(L^{2} / k, 0\right)$.

A matriz jacobiana deste campo no equilíbrio tem traço $-\nu$ e determinante $k^{4} / L^{6}$. E, portanto, autovalores

$$
-\frac{\nu}{2} \pm \sqrt{\frac{\nu^{2}}{4}-\frac{k^{4}}{L^{6}}} .
$$

Logo, essa matriz possui autovalores com parte real negativa em qualquer situação. Ou seja, o ponto de equilíbrio é um atrator hiperbólico.

Nessas novas coordenadas, a energia se escreve $\mathrm{E}=\eta^{2} / 2+L^{2} / 2 r^{2}-k / r$.

É claro que $\mathrm{E}=\eta^{2} / 2+(L /(\sqrt{2} r)-k /(\sqrt{2} L))^{2}-k^{2} /\left(2 L^{2}\right) \geq-k^{2} /\left(2 L^{2}\right)$.

Vejamos como são os níveis (não vazios) dessa função quando assume valores negativos,

$$
\mathrm{E}=-c^{2} \Leftrightarrow \eta^{2}=2 \frac{k}{r}-\frac{L^{2}}{r^{2}}-2 c^{2} .
$$

Seja $f:] 0, \infty\left[\longrightarrow \mathbb{R}, f(r):=\left(2 k r-L^{2}-2 c^{2} r^{2}\right) / r^{2}\right.$. Se $c \neq 0, f$ se anula se e somente se $c^{2} \leq k^{2} /\left(2 L^{2}\right)$, ou seja, para todo $c$ com nível de $\mathrm{E}$ não vazio, com raízes

$$
r_{1,2}=\frac{1}{2 c^{2}}\left(k \pm \sqrt{k^{2}-2 c^{2} L^{2}}\right)
$$

Além disso, $f^{\prime}(r)=2\left(L^{2}-k r\right) / r^{3}$, é monótona decrescente, com raiz $r_{0}=L^{2} / k$. Ainda, sob a hipótese $c^{2} \leq k^{2} /\left(2 L^{2}\right)$, vale

$$
r_{2} \geq \frac{k}{2 c^{2}} \geq \frac{L^{2}}{k}=r_{0}
$$

$\mathrm{E}$, desde que $2 c^{2} r_{2} \geq k$, temos:

$$
r_{1}=\frac{r_{1} r_{2}}{r_{2}}=\frac{L^{2}}{2 c^{2} r_{2}} \leq \frac{L^{2}}{k}=r_{0} .
$$

Portanto, os níveis negativos (não vazios) da energia têm o aspecto representado na figura (2.1). Onde também é destacado o nível de energia nula, facilmente verificado.

Verifica-se que $\nabla \mathrm{E}(r, \eta)=\left(-L^{2} / r^{3}+k / r^{2}, \eta\right)$. E, portanto,

$$
\langle\nabla \mathrm{E}(r, \eta), F(r, \eta)\rangle=-\nu \eta^{2}<0, \quad \forall \eta \neq 0 .
$$

Desse cálculo, vemos que $F(r, \eta)$ é tangente ao nível de energia $\mathrm{E}(r, \eta)$, se e somente se $\eta=0$, $\forall r \in] 0, \infty[$. Caso contrário, o campo $F$ é transversal ao conjunto, garantindo que o seu fluxo por estes pontos, para um intervalo de tempo suficientemente pequeno (e positivo), está contido no interior da curva $\left(\mathrm{E}<\mathrm{E}\left(r_{1}, \eta_{1}\right)\right)$.

Além disso, é óbvio que, dadas duas energias (iniciais) distintas $E_{1}<E_{2}<0$, o conjunto de nível $\mathrm{E}^{-1}\left(E_{1}\right)$ está contido no interior de $\mathrm{E}^{-1}\left(E_{2}\right)$. 


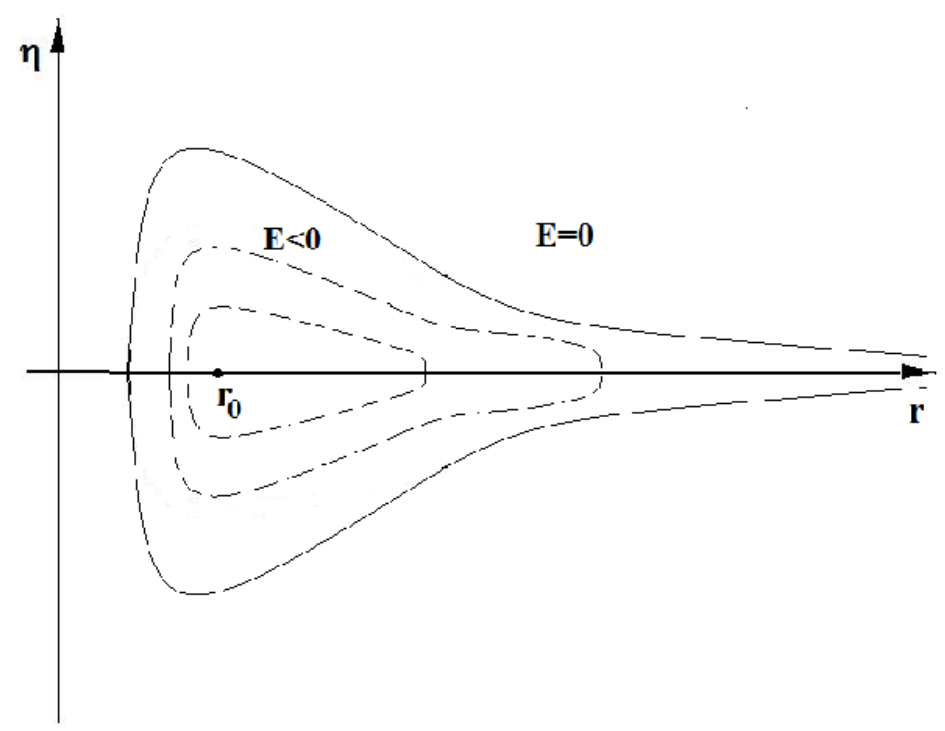

Figura 2.1: Níveis negativos da energia mecânica.

Considere então a solução da equação diferencial com condição inicial $\left(r_{1}, 0\right)$. Neste ponto, $F\left(r_{1}, 0\right)=\left(0,-k / r_{1}^{2}+L^{2} / r_{1}^{3}\right)$, ou seja, é um campo vertical não nulo (para $\left.r_{1} \neq r_{0}\right)$. Logo, existe $\epsilon>0$ suficientemente pequeno tal que $\eta(t) \neq 0, \forall t \in(0, \epsilon)$. Pelo fato da energia ser decrescente ao longo das soluções, $(r(t), \eta(t))$ pertence à união da curva de nível (da energia inicial) e seu interior para todo $t \in(0, \epsilon)$. Mais precisamente, pertence ao interior da curva, pois, se para algum $t \in(0, \epsilon)$, pertencesse ao conjunto de nível, pela transversalidade do campo nesta região haveria uma componente da solução contida no exterior do conjunto de nível. Isto contrariaria a monotonicidade da energia.

Assim, a função energia será estritamente decrescente ao longo de qualquer solução com condição inicial de energia negativa $\left(\mathrm{E}_{0}<0\right)$. Mais do que isto, vemos que nesta situação o fluxo permanecerá em um conjunto compacto, ou seja, as soluções estão definidas para todo $t>0$.

Portanto, concluímos que toda solução com $\mathrm{E}_{0}<0$ tende assintoticamente ao equilíbrio $\left(r_{0}, 0\right)$.

Qualitativamente, a figura (2.2) ilustra o comportamento das soluções nesta região.

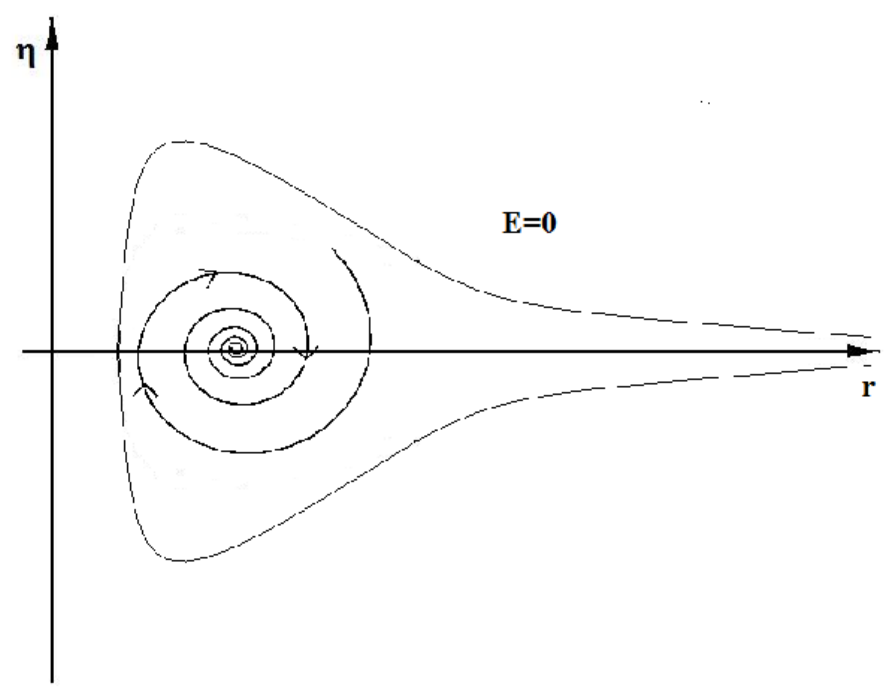

Figura 2.2: Comportamento qualitativo das soluções. 
Na figura, o comportamento das soluções próximas ao equilíbrio, do "tipo espiral", deve-se ao fato de $F$ ter rotacional estritamente negativo no equilíbrio, para quaisquer valores das constantes $k, L$ e $\nu$. Escrevendo o sistema de equações em coordenadas polares em torno do equilíbrio, pode-se afirmar que o ângulo correspondente às trajetórias nesta região tenderá a $\pm \infty$ caso $\nu<2 k^{2} / L^{3}$ (analogamente ao oscilador harmônico subamortecido). Se $\nu>2 k^{2} / L^{3}$, o referido ângulo será assintoticamente limitado (analogamente ao oscilador harmônico superamortecido). Para uma prova dessas afirmações, consultar a seção 30 do capítulo 5 da referência [Pon62].

Observação 2.1.1. Note que no caso sem amortecimento $(\nu=0)$, temos a situação usual, onde a energia E é integral primeira. Ou seja, as soluçôes ficam restritas às curvas esboçadas na figura (2.1). Isso é o esperado, tendo em vista que a solução deste clássico problema (com energia negativa) é o do movimento relativo dos corpos restrito a uma elipse (implicando que a coordenada r seja periódica), ou a um círculo (caso especial em que $r=r_{0}$, ou seja, é a solução de equilíbrio).

Em contrapartida, no caso em consideração, qualquer condição inicial com energia suficientemente baixa (e momento angular não nulo) evoluirá para um estado de rotação do sistema no qual a distância relativa é constante (sem colisão). Em outras palavras, para um observador situado em um dos corpos (referencial não-inercial) o outro corpo descreverá um movimento circular assintoticamente.

É importante ressaltar que este movimento circular existe no caso em que $L \neq 0$. Como nas novas coordenadas, $L=r^{2} \dot{\theta}$, se tivermos inicialmente $L=0$, decorre que $\theta$ é constante (pois $r>0$ ). Logo, as equações de movimento ficam:

$$
\left\{\begin{array}{l}
\dot{r}=\eta \\
\dot{\eta}=-\nu \eta-\frac{k}{r^{2}}
\end{array}\right.
$$

$\mathrm{E}$ assim, desde que $\mathrm{E}=\dot{r}^{2} / 2-k / r$ e $\dot{\mathrm{E}}=-\nu \dot{r}^{2} \leq 0$, por um argumento similar ao anterior, concluímos que as regiões de energia negativas são positivamente invariantes pelo fluxo. Desta forma, dado $\epsilon>0$, e considerando a região $A_{\epsilon}$, compacta, representada na figura (2.3), tome uma solução maximal que "entre"em $A_{\epsilon}$. Se ela não sair de $A_{\epsilon}$, então ela está definida para todo $t \in(0, \infty)$, pois esta região é compacta. Então pelo teorema de Poincaré-Bendixson deveria existir um ponto de equilíbrio ou uma órbita periódica em $A_{\epsilon}$, o que geraria uma contradição, pois não há pontos de equilíbrio e em uma órbita periódica, a energia não poderia ser decrescente.

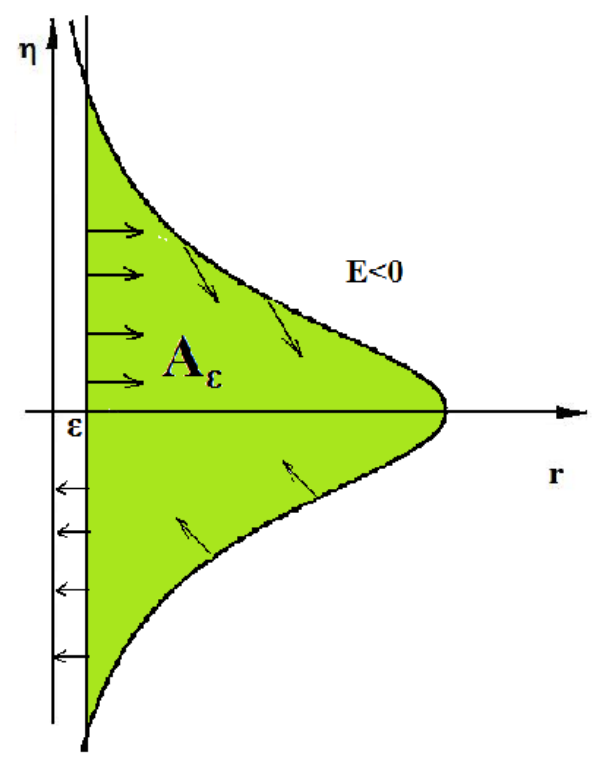

Figura 2.3: Regiões de energia negativa para momento angular nulo. 
Concluímos assim que toda solução (maximal), definida no intervalo $\left(0, t_{0}\right)$, com energia inicial negativa tem a propriedade:

$$
\lim _{t \rightarrow t_{0}} r(t)=0
$$

Ou seja, os dois corpos colidem, assim como no caso conservativo.

Observação 2.1.2. Suponha que $t_{0}=+\infty$. Neste caso, teríamos

$$
\lim _{t \rightarrow+\infty}|\dot{r}|=\lim _{t \rightarrow+\infty} \sqrt{E+\frac{k}{r(t)}}=+\infty .
$$

Logo, existe $N>0$ tal que $|\dot{r}(t)|>1, \forall t>N$. Seja $C:=\max _{0 \leq t \leq N}|\dot{r}(t)|$.

Mas isto implicaria que

$$
|r(t)|=\left|\int_{0}^{t} \dot{r}(s) d s\right| \geq\left|\int_{N}^{t} \dot{r}(s) d s\right|-\left|\int_{0}^{N} \dot{r}(s) d s\right| \geq t-N-C N \stackrel{t \rightarrow \infty}{\longrightarrow} \infty .
$$

Gerando assim um absurdo. Logo, as particulas colidem em tempo finito.

Portanto, para energias iniciais negativas, a presença da dissipação não faz com que os dois corpos colidam (contanto que suas velocidades iniciais não estejam na direção que liga os corpos), o que é intuitivamente plausível.

Observação 2.1.3. O ponto de equilíbrio relativo $(r, \eta)=\left(r_{0}, 0\right)$ tem outra característica interessante. A partir da expressão da energia reduzida (ou seja, com $L=$ cte), tem-se:

$$
\nabla E=\left(-\frac{L^{2}}{r^{3}}+\frac{k}{r^{2}}, \eta\right) \Rightarrow \nabla E\left(r_{0}, 0\right)=(0,0)
$$

Além disso, sua matriz hessiana é:

$$
H_{E}=\left(\begin{array}{cc}
1 & 0 \\
0 & \frac{3 L^{2}}{r^{4}}-\frac{2 k}{r^{3}}
\end{array}\right) \Rightarrow H_{E}\left(r_{0}, 0\right)=\left(\begin{array}{cc}
1 & 0 \\
0 & \frac{k^{4}}{L^{6}}
\end{array}\right) .
$$

Isto implica que $\left(L^{2} / k, 0\right)$ é mínimo local (pelo menos) não degenerado da função energia reduzida. Claramente a solução de equilíbrio desta equação é estável, uma vez que o equilíbrio é atrator hiperbólico.

No entanto, no problema original, onde $L$ não é fixada a priori, as correspondentes soluções circulares não são estáveis (no sentido de Liapounov). Pode-se constatar este fato observando que para quaisquer duas condiçôes iniciais próximas (com momentos angulares distintos) são gerados movimentos circulares (de mesmo raio) com velocidades angulares distintas. Mas isto não as impede de ser orbitalmente estáveis.

Deve-se observar que a força dissipativa aqui usada é extremamente particular. De fato, a determinação experimental de forças dissipativas é algo complexo, e frequentemente recorre-se a expressões simplificadas por razões práticas. No entanto, um ponto que deve ser destacado neste modelo, é que a força utilizada é compatível com o princípio de Galileu para sistemas mecânicos isolados (dentre os quais este se enquadra). Uma discussão sobre este princípio é exposta no Apêndice A.

\subsection{Dissipação interna em um corpo isolado}

A descrição do movimento de corpos cujas propriedades internas e dimensões são não desprezíveis (frente à situação que estão sujeitos) será desenvolvida com mais detalhes, e com maior generalidade, 
no capítulo 3. Nesta seção de motivação, é apresentada uma proposta desenvolvida na referência [LB72].

Um assunto vastamente discutido em textos de mecânica clássica é o corpo rígido, que consiste em um objeto (macroscópico) com uma distribuição de massa não necessariamente homogênea munido de um vínculo interno, qual seja, de que a distância relativa de suas componentes seja constante no tempo. Essa propriedade justifica a ideia de rigidez. O assunto muitas vezes se torna complicado, devido aos tipos de forças (ou torques) externos às quais o objeto está sujeito.

O intuito desta seção é analisar fenomenologicamente os efeitos de dissipação interna de energia em corpos elásticos na ausência de forças externas. Trata-se de algo semelhante ao que foi feito na seção anterior, ou seja, uma tentativa de tornar o modelo mais real ao adicionar um decréscimo gradual na energia mecânica do sistema.

Para uma abordagem construtiva do problema, desde as hipóteses citadas até as equações de movimento, pode-se consultar, por exemplo, o capítulo 6 da referência [Arn89]. Nela, autor deduz as equações de movimento para um sistema constituído de $N$ partículas cujas distâncias mútuas são fixas e no final as "adapta" para o caso contínuo.

Considere um referencial ortonormal de $\mathbb{R}^{3}$ que se move juntamente ao corpo. A hipótese de ausência de forças externas implica que o centro de massa do corpo se move com velocidade constante em relação a qualquer referencial inercial que se tome. Portanto, pode-se tomar este ponto como origem tanto do referencial fixo no corpo (referencial móvel) quanto de um referencial inercial. Neste caso, o movimento do corpo fica determinado pela rotação do referencial móvel em relação ao referencial inercial. Tal rotação é representada por uma função (suave)

$$
Y:[0, \infty) \longrightarrow S O(3),
$$

sendo que a base móvel é, por definição, a imagem da base canônica de $\mathbb{R}^{3}$ por $Y(t)$, para cada $t$.

Desta forma, $Y(t) Y(t)^{T}=I d \Rightarrow \dot{Y} Y^{T}+Y \dot{Y}^{T}=0 \Rightarrow \dot{Y} Y^{T}=-\left(\dot{Y} Y^{T}\right)^{T}$. Ou seja, $\dot{Y} Y^{T}$ é antissimétrica.

Denote por $A n t(3)$ o espaço vetorial dos operadores lineares antissimétricos sobre $\mathbb{R}^{3}$. Assim, usando o isomorfismo $S: \operatorname{Ant}(3) \longrightarrow \mathbb{R}^{3}$ descrito no lema (3.2.3) (que não é apresentado aqui por não ser essencial neste desenvolvimento), podemos definir $\omega_{0}(t):=S\left(\dot{Y}(t) Y(t)^{T}\right) \in \mathbb{R}^{3}$, que é denominado vetor velocidade angular no referencial inercial. Fisicamente, a direção deste vetor é aquela (única, se o corpo não está em repouso) à qual as velocidades instantâneas de todas componentes do corpo são perpendiculares. Seu sentido está associado à orientação da rotação, e sua norma representa o quão rápido está girando o corpo em torno da direção mencionada. Todas estas ideias podem ser estabelecidas de forma matemática, como feito em [Arn89], em termos das velocidades e posições das componentes do corpo. Mas, para o propósito desta motivação, estas ideias são suficientes. Aqui, a parte essencial da discussão é sobre a evolução deste vetor no tempo.

Assumindo que a extensão espacial do corpo é representada por um subconjunto aberto (limitado e conexo) $\mathcal{B} \subset \mathbb{R}^{3}$, tome inicialmente um referencial no centro de massa do corpo. Seja $\rho=\rho\left(x_{1}, x_{2}, x_{3}\right)$ sua densidade de massa e defina o operador linear (conhecido como tensor de inércia no referencial do corpo) $I \in \operatorname{Lin}\left(\mathbb{R}^{3}\right)$, com componentes (em qualquer base ortonormal positiva de $\left.\mathbb{R}^{3}\right)$ :

$$
I_{i j}:=\int_{\mathcal{B}}\left(\left(\sum_{k=1}^{3} x_{k}^{2}\right) \delta_{i j}-x_{i} x_{j}\right) \rho\left(x_{1}, x_{2}, x_{3}\right) d x_{1} d x_{2} d x_{3} .
$$

Desta definição, decorre que $I$ é simétrico e seus autovalores são positivos. Isto é consequência do lema (3.2.5), apresentado posteriormente.

Defina $\omega(t):=Y(t)^{T} \omega_{0}(t)$ e $L:=I \omega$, chamados velocidade $e$ momento angular no referencial do corpo respectivamente. Este último é de fato o momento angular total do sistema descrito no referencial móvel. Pensando no corpo rígido como um sistema de partículas, usa-se o resultado de que na ausência de forças (e portanto torques) externas, o vetor momento angular, no referencial inercial, $\ell:=Y(t) L(t)=Y(t) I \omega(t)=Y(t) I Y(t)^{T} \omega_{0}(t)$, é constante no tempo. 
Desta forma, este vetor satisfaz:

$$
\begin{gathered}
Y I \omega(t)=\ell=\text { constante } \Rightarrow Y I \dot{\omega}+\dot{Y} I \omega=0 \\
\Rightarrow I \dot{\omega}+\left(Y^{T} \dot{Y}\right) I \omega=I \dot{\omega}+Y^{T}\left(\dot{Y} Y^{T}\right) Y I \omega=I \dot{\omega}+Y^{T}\left(\omega_{0} \times Y I \omega\right)=I \dot{\omega}+\omega \times I \omega=0 .
\end{gathered}
$$

Onde foi usada a propriedade (iii) do lema (3.2.3) e o resultado: $A(u \times v)=(A u) \times(A v), \forall u, v \in$ $\mathbb{R}^{3}, \forall A \in S O(3)$.

Portanto, $\omega$ satisfaz a seguinte equação de movimento:

$$
I \dot{\omega}=I \omega \times \omega
$$

Observação 2.2.1. Para ilustração, suponha que o corpo execute um movimento com velocidade angular $\omega_{0}$ constante. Nesta situação, é fácil entender o motivo da interpretação física associada a este vetor. Sob esta hipótese, ainda supondo $\omega_{0}$ não nulo, em uma base ortonormal ordenada de $\mathbb{R}^{3}$ onde o terceiro vetor tem a direção e sentido de $\omega_{0}$, tem-se que $\dot{Y}(t) Y(t)^{T}=S^{-1}\left(\omega_{0}\right)=A_{0}=$ constante, onde:

$$
A_{0}=\left(\begin{array}{ccc}
0 & -a_{3} & a_{2} \\
a_{3} & 0 & -a_{1} \\
-a_{2} & a_{1} & 0
\end{array}\right)=\left(\begin{array}{ccc}
0 & -\left\|\omega_{0}\right\| & 0 \\
\left\|\omega_{0}\right\| & 0 & 0 \\
0 & 0 & 0
\end{array}\right) .
$$

Assim, $\dot{Y}=A_{0} Y$, e portanto,

$$
Y=Y_{0} \exp \left(t A_{0}\right)=Y_{0}\left(\begin{array}{ccc}
\cos \left(\left\|\omega_{0}\right\| t\right) & -\operatorname{sen}\left(\left\|\omega_{0}\right\| t\right) & 0 \\
\operatorname{sen}\left(\left\|\omega_{0}\right\| t\right) & \cos \left(\left\|\omega_{0}\right\| t\right) & 0 \\
0 & 0 & 1
\end{array}\right) .
$$

Logo, o referencial móvel girará em torno da direção determinada por $Y_{0} \omega_{0}$ com velocidade angular igual ao módulo deste vetor. Além disso, $0=\dot{\omega}_{0}=\dot{Y} \omega+Y \dot{\omega}=A_{0} Y \omega+Y \dot{\omega}$. Mas, novamente pela propriedade (iii) do lema (3.8.3), $A_{0} Y \omega=S^{-1}\left(\omega_{0}\right) \omega_{0}=\omega_{0} \times \omega_{0}=0$. Portanto, $Y \dot{\omega}=0 \Rightarrow \omega=$ constante.

Suponha agora que a velocidade angular no referencial do corpo, $\omega$, seja constante no tempo $e$ não nula. Pela equação de movimento, obtemos que $0=I \dot{\omega}=(I \omega) \times \omega$. Logo $I \omega=\lambda \omega, \lambda \in \mathbb{R}$, $\lambda \neq 0$. Logo $\ell=Y I \omega=\lambda Y \omega \Rightarrow \omega_{0}=Y \omega=\lambda^{-1} \ell$. Assim, $\omega_{0}$ também é constante, e o movimento é o mesmo descrito anteriormente. Além disto, o cálculo mostrou que ele tem a mesma direção que o momento angular $\ell$ (consequentemente, $\omega \sim L$ ).

Ou seja, $\omega_{0}$ é constante se e somente se $\omega$ também for, tornando equivalentes as questões de encontrar rotações estacionárias nos referenciais inercial e do corpo.

A partir da construção apresentada, são diretamente obtidas quatro integrais primeiras, quais sejam, energia mecânica e vetor momento angular:

$$
\ell=Y L=Y I \omega, \quad T=\frac{1}{2}<\omega, I \omega>=\frac{1}{2}<\omega_{0}, Y I Y^{T} \omega_{0}>
$$

Seja $\left\{f_{1}, f_{2}, f_{3}\right\}$ uma base (ortonormal) de autovetores de $I$. Se $\omega_{1}, \omega_{2}, \omega_{3}$ são as coordenadas de $\omega$ nesta base, as quantidades conservadas acima implicam que:

$$
\left\{\begin{array}{c}
\|\ell\|^{2}=I_{1}^{2} \omega_{1}^{2}+I_{2}^{2} \omega_{2}^{2}+I_{3}^{2} \omega_{3}^{2}=\text { constante } \\
2 E=I_{1} \omega_{1}^{2}+I_{2} \omega_{2}^{2}+I_{3} \omega_{3}^{2}=\text { constante }
\end{array}\right.
$$

Ou seja, no referencial do corpo, a velocidade angular se move ao longo da interseção de dois elipsóides. Esta interseção somente será bidimensional no caso $I_{1}=I_{2}=I_{3}\left(\mathrm{e}\|\ell\|^{2}=2 I_{1} E\right.$ ), onde $\omega$ é constante. Quando os momentos de inércia são mutuamente distintos, pode-se notar que existem somente seis pontos de equilíbrio do fluxo sobre cada superfície de momento angular constante. Portanto, conclui-se que existem somente três tipos de trajetórias da velocidade angular possíveis: restrita a uma órbita periódica, constante em um ponto de equilíbrio e em uma conexão de selas. 
A figura (2.4), extraída da referência [BO78], ajuda a compreender esta situação.

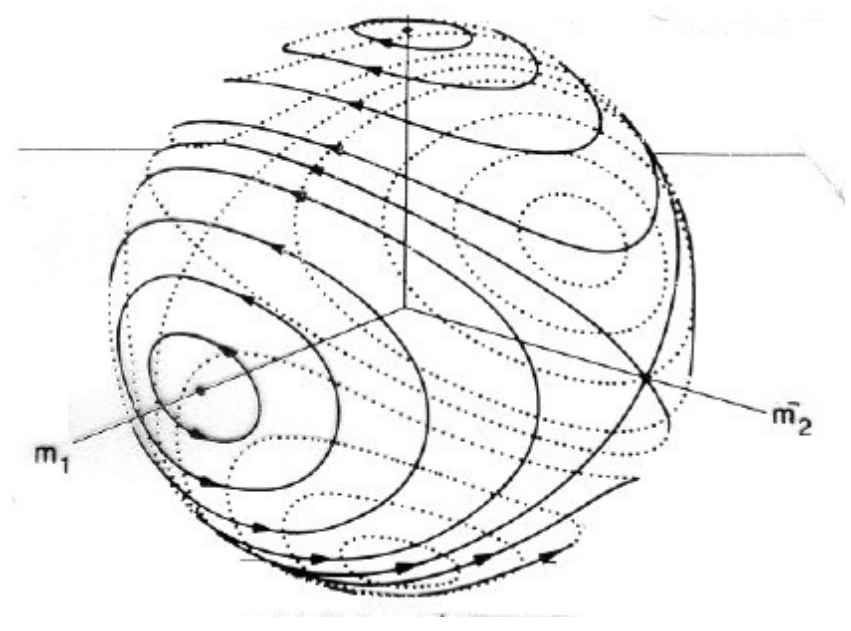

Figura 2.4: Retrato de fase para a velocidade angular no referencial do corpo rígido.

Observa-se que os pontos de equilíbrio ao longo dos eixos de maior e menor inércia são estáveis (no sentido de Liapounov), ao contrário do equilíbrio no eixo de inércia intermediária.

É fácil constatar que no caso em que (somente) dois dos momentos de inércia são iguais (para um sólido de revolução, por exemplo), as conexões de sela deixam de existir e surge uma curva fechada constituída de pontos de equilíbrio.

Considerando então o caso menos degenerado (representado na figura), pergunta-se o que aconteceria com as trajetórias sobre o elipsóide de momento angular constante se a energia do sistema decrescesse gradualmente.

Esta proposta baseia-se no fato de que usualmente corpos sólidos na natureza não têm seu movimento bem descrito pela dinâmica do corpo rígido. Este modelo fica particularmente ineficaz em grandes escalas de tempo, isto é, para descrever o movimento assintótico. Corpos sólidos isolados em rotação têm suas componentes submetidas a aceleração, o que causa deformações, vibrações. A energia mecânica passa a ser transferida para estes movimentos internos do corpo, não tão lentamente, dependendo da geometria do mesmo. Será assumido que a energia mecânica é decrescente, ou seja, que a energia transferida para os movimentos internos não pode ser convertida novamente em rotação do sistema total e que o processo de transferência seja muito lento comparado à velocidade de rotação, o que geralmente é denominado processo quasi-estático. Um bom exemplo real são sólidos que possuem hastes agregadas, as quais vibram com considerável amplitude quando submetidas a rotação.

Os referidos movimentos internos, no entanto, não são suficientes para alterarem o momento angular total. Para que isto ocorra, as deformações têm que imprimir um "torque efetivo"não nulo sobre o corpo, o que será assumido ter muito menos influência do que a dissipação de energia (ou não ocorrer de fato).

Para entender o efeito da dissipação de energia no movimento do corpo, analisaremos o que ocorre próximo aos movimentos de "equilíbrio", onde $\omega$ é constante.

Pensando de forma intuitiva, dada uma velocidade angular inicial próxima a qualquer um dos dois equilíbrios de menor momento de inércia, o correspondente nível de energia está próximo do maior admissível (em relação ao momento angular correspondente, fixo). Desta forma, a velocidade angular descreverá aproximadamente uma curva fechada, que não poderá ser periódica devido à leve queda na energia. E o movimento evolui desta forma na sequência, afastando-se do equilíbrio, até se aproximar da região onde existiam as conexões de sela. Ou seja, nestas regiões o equilíbrio atua como um repulsor, com comportamento semelhante a um foco repulsor linear. 
Nas regiões próximas aos equilíbrios de maior momento de inércia, na região que era compreendida entre as conexões de sela, tem-se um comportamento análogo, porém o equilíbrio passaria a ser um atrator das órbitas do correspondente setor. Em outras palavras, toda rotação com velocidade angular próxima tenderia a uma rotação estacionária, uma configuração de energia mínima, na qual se cessaria a deformação do corpo e, consequentemente, a dissipação.

Ao ponto de sela, é atribuída uma idéia de robustez, sendo que ele permaneceria na presença da "perturbação" imposta. No entanto, as conexões de sela seriam provavelmente desfeitas.

Assim, após esta série de afirmações que parecem razoáveis, é sugerido que não há alternativa senão aquela em que, exceto nos pontos de equilíbrio instáveis e nas separatrizes de sela estáveis, toda velocidade angular inicial evoluirá para uma rotação ao longo do eixo de maior momento de inércia.

A figura (2.5), retirada da referência [BL87], ilustra a situação descrita.

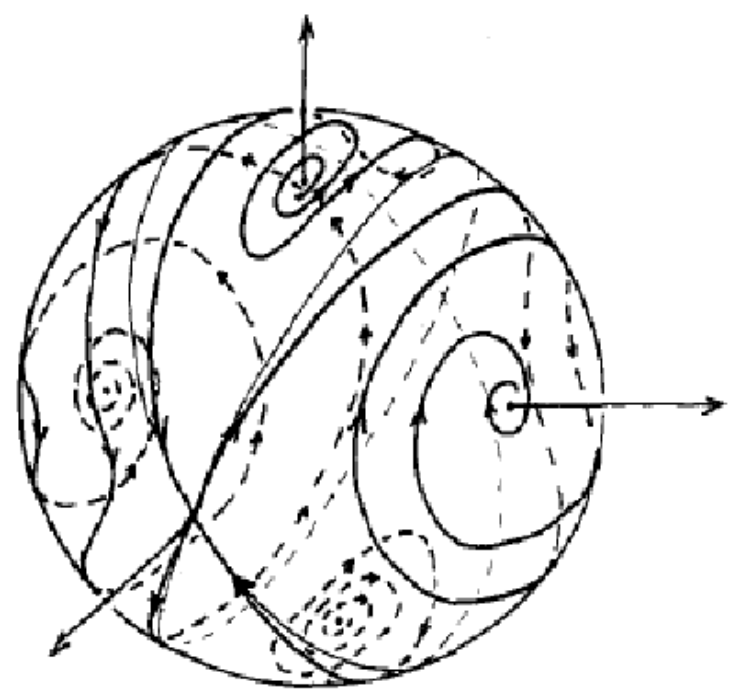

Figura 2.5: Retrato de fase para a velocidade angular no referencial do corpo com dissipação.

Sendo assim, é interessante entender o significado físico deste estado.

Pela observação (2.2.1), para uma rotação estacionária: $\omega=Y(t)^{T} \omega_{0}=$ constante. Logo, naquela base citada,

$$
Y_{0}^{T} \omega_{0}=\left(\begin{array}{ccc}
\cos \left(\left\|\omega_{0}\right\| t\right) & \operatorname{sen}\left(\left\|\omega_{0}\right\| t\right) & 0 \\
-\operatorname{sen}\left(\left\|\omega_{0}\right\| t\right) & \cos \left(\left\|\omega_{0}\right\| t\right) & 0 \\
0 & 0 & 1
\end{array}\right) \omega
$$

Assim, pela independência linear das funções seno e cosseno, conclui-se que $\omega=(0,0, \pm\|\omega\|)=$ $\left(0,0, \pm\left\|\omega_{0}\right\|\right)$.

Logo,

$\left(\begin{array}{c}0 \\ 0 \\ \left\|\omega_{0}\right\|\end{array}\right)=Y_{0}\left(\begin{array}{c}0 \\ 0 \\ \pm\left\|\omega_{0}\right\|\end{array}\right) \Rightarrow Y_{0}=\left(\begin{array}{ccc}\cos \alpha & \operatorname{sen} \alpha & 0 \\ -\operatorname{sen} \alpha & \cos \alpha & 0 \\ 0 & 0 & 1\end{array}\right),\left(\begin{array}{ccc}\operatorname{sen} \alpha & \cos \alpha & 0 \\ \cos \alpha & -\operatorname{sen} \alpha & 0 \\ 0 & 0 & -1\end{array}\right)$.

Onde, na última implicação, foi usado que obrigatoriamente $Y_{0} \in S O(3)$.

$\mathrm{E}$, finalmente,

$$
Y(t)=\left(\begin{array}{ccc}
\cos \left(\left\|\omega_{0}\right\| t+\alpha\right) & \operatorname{sen}\left(\left\|\omega_{0}\right\| t+\alpha\right) & 0 \\
-\operatorname{sen}\left(\left\|\omega_{0}\right\| t+\alpha\right) & \cos \left(\left\|\omega_{0}\right\| t+\alpha\right) & 0 \\
0 & 0 & 1
\end{array}\right)
$$


Portanto, segundo o referencial inercial, assintoticamente, quase todo estado inicial evolui para uma rotação "bem comportada" do corpo, na qual todas suas componentes (ou "pontos materiais" que o constituem) descrevem um movimento circular, com o módulo da velocidade constante, em torno do eixo de maior inércia, que coincide neste caso com a direção da velocidade angular "fixa no espaço".

Esta foi a motivação para estudar o corpo rígido no contexto de dissipação de energia, e conservação do momento angular. Este modelo tem analogias muito interessantes com relação ao apresentado na seção (2.1). Além da questão das duas integrais primeiras citadas, outra analogia reside no movimento assintótico, o qual é estável e está na interseção do nível de momento angular constante e mínimo local relativo de energia (como discutido na observação (2.1.3)). Ainda, em ambos os movimentos assintóticos, o sistema está em um equilíbrio que não é "estático", isto é, as componentes do mesmo continuam em movimento em relação a qualquer referencial inercial (se o momento angular total é não nulo).

Note que ambas as situações foram interessantes quanto à conservação do momento angular, que facilitou extremamente a análise reduzindo os graus de liberdade do sistema essencialmente para dois, contexto no qual a teoria qualitativa das equações diferenciais é muito mais acessível.

\subsection{Oscilador harmônico amortecido acoplado a corpo rígido livre}

Desde que a última seção foi dedicada a uma abordagem intuitiva sobre dissipação em sólidos, propõe-se agora uma situação (talvez a mais simples possível) deste tipo de mecanismo.

O estudo do modelo apresentado aqui foi proposto e desenvolvido na referência [Lev89]. Todos os resultados aqui expostos foram apresentados na mesma.

Considere um corpo rígido, com operador de inércia $I$, cujos momentos de inércia são $I_{1} \geq I_{2} \geq$ $I_{3}$. Ao longo do eixo de maior inércia, estão acopladas ao corpo duas partículas de massas iguais $\left(m_{p}\right)$ através de molas com elasticidade, amortecimento e comprimento iguais. As partículas estão vinculadas a se movimentar somente ao longo deste eixo, sendo que as molas estão orientadas em sentidos contrários. A figura (2.6) ilustra a situação.

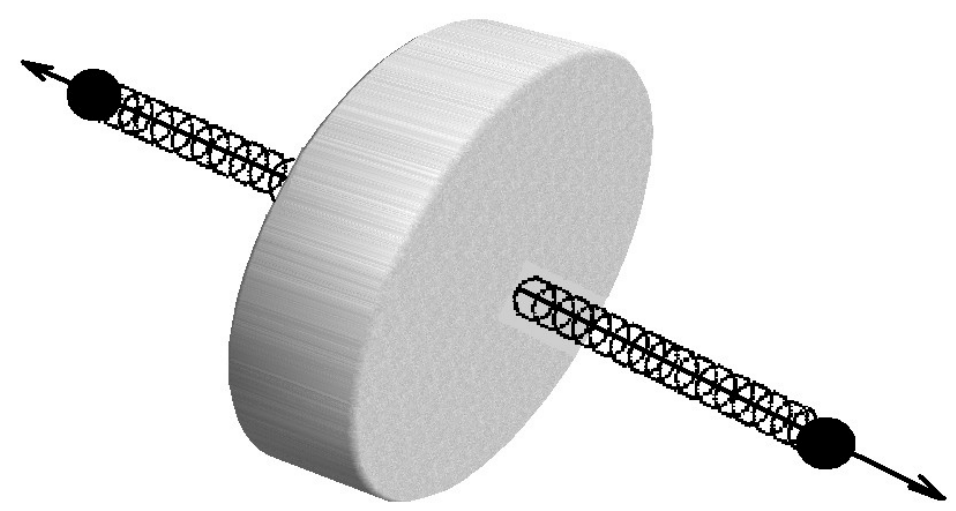

Figura 2.6: Corpo rígido acoplado a oscilador harmônico amortecido.

Observe que se $I_{1}=I_{2}$, o eixo em questão não é único. Escolhendo um, as equações de movimento a seguir também serão válidas.

Escolha uma base ortonormal de autovetores de $I$, e denomine por eixos $x, y, z$ os associados aos momentos $I_{1}, I_{2}, I_{3}$, respectivamente. Este é um referencial fixo no corpo, sendo que as duas molas oscilam ao longo do eixo $\mathrm{x}$. 
Sejam $k$ a constante de mola, $\pm x_{0}$ as posições de equilíbrio das partículas e $c$ a constante de amortecimento das molas.

Pensando no modelo como um sistema de partículas, e assumindo que não haja força externa, faz-se uso da conservação do momento angular total para encontrar as equações de movimento (este pode ser pensado como obtido a partir da mecânica Newtoniana ou através do teorema de Noether).

Para simplificar o problema, suponha que as condições iniciais das partículas sejam simétricas, de forma que possamos assumir que o centro de massa do sistema esteja em repouso em relação ao corpo rígido.

Desta maneira, o momento angular total do sistema é dado por:

$$
\ell:=\sum_{i} m_{i} r_{i} \times v_{i}=\ell_{C R}+2 m_{p} r \times v
$$

Onde $\ell_{C R}=Y(t) I \omega$ é o momento angular do corpo rígido, $r, v$ são a posição e a velocidade da partícula de coordenada $x$ positiva (a posição e a velocidade da outra são simétricas a $r$ e $v, \operatorname{logo}$ $\left.r_{2} \times v_{2}=r \times v\right)$.

Sendo $Y(t)$ como discutido na seção anterior, e pela descrição do sistema, vemos que $r=$ $Y(t)(x \hat{x})=x Y(t) \hat{x}$. Onde $x=\|r\|$ e $\hat{x}$ é o vetor unitário na direção positiva do eixo $x$.

Logo, $v=\dot{r}=\dot{x} Y \hat{x}+x \dot{Y} \hat{x}=\dot{x} Y \hat{x}+x \omega_{0} \times(Y \hat{x}) \Rightarrow r \times v=x Y \hat{x} \times\left(x \omega_{0} \times Y \hat{x}\right)=Y\left(x^{2} \hat{x} \times(\omega \times \hat{x})\right)$.

Portanto, $\ell=Y M$, onde

$$
\begin{gathered}
M=I \omega-2 m_{p} x^{2} \hat{x} \times(\hat{x} \times \omega) . \\
\Rightarrow 0=\dot{\ell}=Y \dot{M}+\dot{Y} Y^{T} Y M=Y \dot{M}+\omega_{0} \times Y M \\
\Rightarrow \dot{M}+\omega \times M=0 .
\end{gathered}
$$

Além disso, as duas partículas estão sujeitas à interação com o corpo, através das molas. Para descrever esta interação, escreve-se a equação de movimento (Newtoniana) para as mesmas:

$$
m_{p} \dot{v}=F=-k\left(x-x_{0}\right) Y \hat{x}-c \dot{x} Y \hat{x}+F_{v i n c} .
$$

Onde $F_{v i n c}$ é a força de vínculo associada à restrição de que a partícula se movimente somente na direção do eixo $x$.

Assumindo-se o princípio de D'Alembert, infere-se que a componente de $F_{\text {vinc }}$ na direção de $\hat{x}$ é nula.

Mas, $v=Y(x \omega \times \hat{x}+\dot{x} \hat{x}) \Rightarrow \dot{v}=Y(\ddot{x} \hat{x}+x \omega \times(\omega \times \hat{x})+2 \dot{x} \omega \times \hat{x}+x \dot{\omega} \times \hat{x})$.

Logo as equações de movimento são:

$$
\left\{\begin{array}{c}
\dot{M}+\omega \times M=0 \\
m_{p} \ddot{x}+k\left(x-x_{0}\right)+c \dot{x}+m_{p} x<\omega \times(\omega \times \hat{x}), \hat{x}>+2 m_{p}<\dot{x} \omega \times \hat{x}, \hat{x}>+m_{p} x<\dot{\omega} \times \hat{x}, \hat{x}>=0
\end{array}\right.
$$

Ou, explicitamente em termos das coordenadas de $\omega$ na base citada:

$$
\left\{\begin{array}{c}
I \dot{\omega}+\omega \times I \omega+4 m_{p} x \dot{x}\left(0, \omega_{2}, \omega_{3}\right)+2 m_{p} x^{2}\left(0, \dot{\omega}_{2}, \dot{\omega}_{3}\right)+2 m_{p} x^{2} \omega_{1}\left(0,-\omega_{3}, \omega_{2}\right)=0 \\
\ddot{x}+k\left(x-x_{0}\right) / m_{p}+c \dot{x} / m_{p}=x\left(\omega_{2}^{2}+\omega_{3}^{2}\right)
\end{array}\right.
$$

Na seção (4.3) este sistema será deduzido usando-se o formalismo Lagrangiano, indicando a compatibilidade do princípio de D'Alembert usado aqui.

É interessante associar uma energia a este sistema. Podemos pensar que a energia total é a soma da energia cinética do corpo rígido, a energia cinética das massas e o termo de interação dado pela energia potencial da mola.

Somos levados assim a definir:

$$
E=\frac{1}{2}<I \omega, \omega>+\frac{1}{2} m_{p}\left(\dot{x}^{2}+\left(\omega_{2}^{2}+\omega_{3}^{2}\right) x^{2}\right)+k\left(x-x_{0}\right)^{2} .
$$

Usando as duas primeiras equações de (2.4) e derivando a energia com respeito ao tempo, 
concluímos por um cálculo direto que $\dot{E}=-2 c \dot{x}^{2} \leq 0$. Isso indica certa adequação do modelo ao contexto proposto. Pois foi visto que o momento angular total é conservado (aqui, praticamente por princípio) e esta função energia é decrescente ao longo das soluções. Observe que na ausência da dissipação, $c=0$, a energia é conservada, e ela também é constante quando é cessado o movimento da mola.

Escrito na base móvel, o momento angular fica:

$$
M=\left(I_{1} \omega_{1},\left(I_{2}+2 m_{p} x^{2}\right) \omega_{2},\left(I_{3}+2 m_{p} x^{2}\right) \omega_{3}\right)=I(x) \omega .
$$

Onde $I(x)=\operatorname{diag}\left(I_{1}, I_{2}(x), I_{3}(x)\right)=\operatorname{diag}\left(I_{1},\left(I_{2}+2 m_{p} x^{2}\right),\left(I_{3}+2 m_{p} x^{2}\right)\right)$, pode ser pensado como um operador de inércia do sistema completo (note que não é necessariamente constante).

A conservação do momento angular implica que é conservado:

$$
\|M\|^{2}=I_{1}^{2} \omega_{1}^{2}+\left(I_{2}+2 m_{p} x^{2}\right)^{2} \omega_{2}^{2}+\left(I_{3}+2 m_{p} x^{2}\right)^{2} \omega_{3}^{2} .
$$

Na referência [Lev89] em questão, o autor afirma que esta é a única quantidade conservada não trivial na presença da dissipação $(c \neq 0)$.

O decréscimo da energia implica que sobre uma solução com energia inicial $E_{0}$ :

$$
0 \leq I_{1} \omega_{1}^{2}+I_{2} \omega_{2}^{2}+I_{3} \omega_{3}^{2}+m_{p}\left(\dot{x}^{2}+\left(\omega_{2}^{2}+\omega_{3}^{2}\right) x^{2}\right)+k\left(x-x_{0}\right)^{2} \leq 2 E_{0} .
$$

Claramente, o conjunto dos pontos $\left(\omega_{1}, \omega_{2}, \omega_{3}, x, \dot{x}\right) \in \mathbb{R}^{5}$ que satisfazem esta desigualdade é limitado, e também fechado, sendo imagem inversa de fechado por função contínua. Logo é compacto.

Além disso, as equações do sistema (2.4) podem ser escritas em forma normal:

$$
\left(\begin{array}{c}
\dot{\omega}_{1} \\
\dot{\omega}_{2} \\
\dot{\omega}_{3} \\
\dot{x} \\
\dot{u}
\end{array}\right)=\left(\begin{array}{c}
\frac{\left(I_{2}-I_{3}\right)}{I_{1}} \omega_{2} \omega_{3} \\
\frac{1}{I_{2}+2 m_{p} x^{2}}\left(\left(I_{3}-I_{1}+2 m_{p} x^{2}\right) \omega_{1} \omega_{3}-4 m_{p} x u \omega_{2}\right) \\
\frac{1}{I_{3}+2 m_{p} x^{2}}\left(\left(I_{1}-I_{2}-2 m_{p} x^{2}\right) \omega_{1} \omega_{2}-4 m_{p} x u \omega_{3}\right) \\
u \\
-k\left(x-x_{0}\right) / m_{p}-c u / m_{p}+x\left(\omega_{2}^{2}+\omega_{3}^{2}\right)
\end{array}\right)
$$

Logo, a solução do sistema para qualquer condição inicial está definida para todo $t>0$. Assim faz sentido investigar o comportamento assintótico das mesmas, de uma forma global.

Daqui em diante, será analisada a solução do sistema (2.9), ou equivalentemente, a do sistema (2.4).

Teorema 2.3.1. Se c $>0$, o conjunto $\omega$-limite de toda solução do sistema está contido em:

$$
\left\{(x, u, \omega) \in \mathbb{R}^{5}: u=0\right\} .
$$

Denominado conjunto de dissipação zero, ou configurações rígidas. Mais do que isto, $\lim _{t \rightarrow \infty} \dot{x}=0$.

Demonstração. Foi visto que toda solução está definida para todo $t \geq 0$, e está contida (positivamente) em um compacto. Um resultado de EDO (ver por exemplo [Sot79], Teorema 5 do capítulo VII) que seu conjunto $\omega$-limite é não vazio, compacto, conexo e invariante pelo fluxo.

Para garantir o teorema, basta mostrar que $\forall \varepsilon \geq 0$, e qualquer condição inicial $Z_{0}=\left(x(0), \dot{x}(0), \omega_{0}\right)$, existe $T=T\left(\varepsilon, Z_{0}\right)$ tal que $\forall t>T, \phi\left(t, Z_{0}\right)=(x(t), \dot{x}(t), \omega(t)) \in\{(x, u, \omega):|u|<\varepsilon\}$.

Suponha que isto não valha. Assim, existem $0<\varepsilon<1, Z_{0}$ e uma sequência de tempos, $t_{1}<$ $t_{2}<\ldots<t_{n}<\ldots$, com $t_{n} \stackrel{n \rightarrow \infty}{\longrightarrow}+\infty$ tais que $\left|\dot{x}\left(t_{n}\right)\right| \geq \varepsilon, \forall n \in \mathbb{N}$.

Ainda, se $|\dot{x}(t)| \geq \varepsilon, \dot{E}(t)=-2 c \dot{x}(t)^{2} \leq-2 c \varepsilon^{2}$. Logo, o conjunto $\{t>0:|\dot{x}(t)| \geq \varepsilon\} \subset \mathbb{R}$ deve ter medida nula. Pois caso contrário, teríamos $E(t) \stackrel{t \rightarrow \infty}{\longrightarrow}-\infty$, absurdo, pois $E \geq 0$. 
Observe que esta conclusão também vale para $\{t>0:|\dot{x}(t)| \geq \varepsilon / 2\}$.

Assim, não há perda de generalidade em assumir que $\forall n \in \mathbb{N}$, existem $t_{n, 0}, t_{n, 1} \in\left(t_{n}, t_{n+1}\right), t_{n, 0}<$ $t_{n, 1}$, tais que $\varepsilon / 2 \leq|\dot{x}(t)| \leq \varepsilon, \forall t \in\left(t_{n, 0}, t_{n, 1}\right),\left|\dot{x}\left(t_{n, 0}\right)\right|=\varepsilon / 2$ e $\left|\dot{x}\left(t_{n, 1}\right)\right|=\varepsilon$.

$$
\Rightarrow E\left(t_{n, 0}\right)-E\left(t_{n, 1}\right)=-\int_{t_{n, 0}}^{t_{n, 1}} \dot{E} d t=2 \int_{t_{n, 0}}^{t_{n, 1}} c \dot{x}^{2} d t \geq c \varepsilon^{2}\left(t_{n, 1}-t_{n, 0}\right) .
$$

Por outro lado,

$$
\frac{\varepsilon}{2}=\left|\dot{x}\left(t_{n, 1}\right)-\dot{x}\left(t_{n, 0}\right)\right| \leq \max _{t_{n, 0} \leq t \leq t_{n, 1}}|\ddot{x}(t)|\left|t_{n, 1}-t_{n, 0}\right| .
$$

No entanto, pela segunda equação de (2.4) e pela equação (2.8), vemos que

$$
|\ddot{x}| \leq \frac{k}{m_{p}}\left|x-x_{0}\right|+\frac{c}{m_{p}}|\dot{x}|+|x|\left(\omega_{2}^{2}+\omega_{3}^{2}\right) \leq K, \forall t>0 .
$$

Onde $K>0$ só depende da energia $E_{0}$.

$$
\begin{gathered}
\Rightarrow E\left(t_{n, 0}\right)-E\left(t_{n, 1}\right) \geq \frac{c \varepsilon^{3}}{4 K} . \\
\Rightarrow \lim _{t \rightarrow \infty} E(t)-E_{0}=-2 c \int_{0}^{\infty} \dot{x}^{2} d t \leq-2 c \sum_{n=1}^{\infty} \int_{t_{n, 0}}^{t_{n, 1}} \dot{x}^{2} d t=-\sum_{n=1}^{\infty}\left(E\left(t_{n, 0}\right)-E\left(t_{n, 1}\right) \leq-\sum_{n=1}^{\infty} \frac{c \varepsilon^{3}}{4 K}=-\infty .\right.
\end{gathered}
$$

Absurdo.

O teorema assegura que para toda condição inicial, assintoticamente, a velocidade das molas vai a zero. Consequentemente, dada qualquer condição inicial sobre o $\omega$-limite de qualquer solução, não haverá dissipação de energia. Isto é compatível com as considerações da seção (2.2).

No entanto, observe o caso em que o corpo possui a simetria $I_{2}=I_{3}$. Por exemplo, quando o corpo é parecido com o da figura (2.6). Das equações (2.9), vemos que existem condições iniciais adequadas que resultam em uma solução do tipo:

$$
\omega_{1}=\text { constante }, \quad x=\text { constante }, \quad \omega_{2}=A \cos (\alpha t), \omega_{3}=A \operatorname{sen}(\alpha t) .
$$

Ou seja, o corpo precessa. Em vista da seção anterior, este é um comportamento inesperado. Esperar-se-ia que todo movimento tendesse a uma rotação em torno do eixo de maior inércia. Mais do que isto, na referência [Lev89], afirma-se que este tipo de solução seria limite assintótico de soluções com outras condições iniciais (até com movimento inicial da mola). No entanto, argumentase que este comportamento é excepcional, e que se deve a uma imposição "artificial" deste modelo, o vínculo das partículas se moverem somente sobre o eixo $x$. Ainda assim, ele afirma que esta precessão assintótica é não genérica, em vista do seguinte resultado.

Teorema 2.3.2. Suponha que $I_{2}>I_{3}$. A curva $(\omega(t), x(t), \dot{x}(t))=\left(\omega(t), x_{\infty}, 0\right)$, onde $x_{\infty}>0$ é constante, é solução do sistema (2.4) se e somente se $\omega(t)=\omega_{\infty}$, constante, com componentes satisfazendo $\omega_{2}^{2}+\omega_{3}^{2}<k / m_{p}$. Neste caso, $x_{\infty}, \omega_{\infty}$ satisfazem:

$$
I\left(x_{\infty}\right) \omega_{\infty}=\lambda \omega_{\infty}, \quad x_{\infty}=\frac{k x_{0}}{k-m_{p}\left(\omega_{2}^{2}+\omega_{3}^{2}\right)} .
$$

I(x) foi definido na equação (2.6).

Demonstração. Sob estas condições, a função $\omega$ satisfaz,

$$
\left\{\begin{array}{c}
\dot{\omega}_{1}=\alpha_{1} \omega_{2} \omega_{3} \\
\dot{\omega}_{2}=\alpha_{2} \omega_{1} \omega_{3} \\
\dot{\omega}_{3}=\alpha_{3} \omega_{1} \omega_{2} \\
x_{\infty}\left(k-m_{p}\left(\omega_{2}^{2}+\omega_{3}^{2}\right)\right)=k x_{0}
\end{array}\right.
$$


Onde $\alpha_{1}:=\left(I_{2}-I_{3}\right) / I_{1}=\left(I_{2}\left(x_{\infty}\right)-I_{3}\left(x_{\infty}\right)\right) / I_{1}, \alpha_{2}:=\left(I_{3}\left(x_{\infty}\right)-I_{1}\right) / I_{2}\left(x_{\infty}\right), \alpha_{3}:=\left(I_{1}-\right.$ $\left.I_{2}\left(x_{\infty}\right)\right) / I_{3}\left(x_{\infty}\right)$.

Note que a última equação impõe que a solução $\omega(t)$ está restrita a um cilindro. Observando a conservação do momento angular e da energia, para provar que $\omega$ é constante basta mostrar que na interseção deste cilindro com os dois correspondentes elipsóides não pode conter um segmento de curva.

A última equação fornece: $0=(d / d t)\left(\omega_{2}^{2}+\omega_{3}^{2}\right)=\left(\alpha_{2}+\alpha_{3}\right) \omega_{1} \omega_{2} \omega_{3}$.

Suponha que $\alpha_{2}=-\alpha_{3}$. Daí temos que:

$$
\begin{gathered}
\frac{I_{3}+2 m_{p} x_{\infty}-I_{1}}{I_{3}+2 m_{p} x_{\infty}}>\frac{I_{3}+2 m_{p} x_{\infty}-I_{1}}{I_{2}+2 m_{p} x_{\infty}}=\frac{I_{2}+2 m_{p} x_{\infty}-I_{1}}{I_{3}+2 m_{p} x_{\infty}} \\
\Rightarrow I_{3}>I_{2} .
\end{gathered}
$$

Gerando um absurdo. Logo, $\omega_{1} \omega_{2} \omega_{3} \equiv 0$.

Suponha que existam $t_{0}$ e $i$ tais que $\dot{\omega}_{i} \neq 0$. Logo, isto vale em uma vizinhança de $t_{0}$. Logo,

$$
0=\alpha_{i} \omega_{1} \omega_{2} \omega_{3}=\omega_{i} \frac{d}{d t} \omega_{i}=\frac{d}{d t} \omega_{i}^{2} .
$$

Portanto, $\left|\omega_{i}\right|$ é constante nesta vizinhança. O que é absurdo. Conclui-se então que $\omega$ é constante.

A última equação fornece então o valor que $x_{\infty}$ deve ter, o qual está bem definido se atendida a hipótese.

Observe que a primeira equação de (2.4) agora mostra que: $0=\omega \times M \omega=\omega \times I\left(x_{\infty}\right) \omega$. Ou seja, $I\left(x_{\infty}\right) \omega=\lambda \omega$.

No próximo teorema, será imposto $m_{p}=1$, sem que haja perda na análise qualitativa.

Teorema 2.3.3. Suponha que os momentos de inércia e o comprimento de relaxamento da mola satisfaçam: $I_{1}>I_{2}\left(x_{0}\right)>I_{3}\left(x_{0}\right)$. Seja $\mu:=\|M\|$, a norma do momento angular inicial (que é conservada). Considere o valor da constante de mola $k$ no seguinte intervalo:

$$
0<k<\frac{\mu}{I_{1}^{2}}\left(1-\frac{x_{0}}{\sqrt{I_{1}-I_{2}}}\right)^{-1}=: k_{y} .
$$

Para cada um destes valores, existem dois eixos no plano $(x, y), y^{\prime}$ e $y^{\prime \prime}$, diferentes dos eixos $x$ e y, tais que o sistema admite uma rotação estacionária em torno deles. Ou seja, $\dot{\omega}=0$, e $\omega$ está na direção do eixo.

$O$ análogo deste resultado vale no plano $(x, z)$, com eixos $z^{\prime}$ e $z^{\prime \prime}$, e

$$
k_{z}:=\frac{\mu}{I_{1}^{2}}\left(1-\frac{x_{0}}{\sqrt{I_{1}-I_{3}}}\right)^{-1} .
$$

Demonstração. De fato, note que os únicos equilíbrios possíveis do sistema $(2.9)$ são $\left(\omega_{1}, \omega_{2}, \omega_{3}, x, \dot{x}\right)=$

(i) $\left( \pm \mu / I_{1}, 0,0, x_{0}, 0\right)$.

(ii) $\left(0, \pm \mu / I_{2}(x), 0, x, 0\right)$. Onde $x$ satisfaz: $k\left(I_{2}+x^{2}\right)^{2}\left(x-x_{0}\right)=\mu^{2} x$.

(iii) $\left(0,0, \pm \mu / I_{3}(x), x, 0\right)$. Onde $x$ satisfaz: $k\left(I_{2}+x^{2}\right)^{2}\left(x-x_{0}\right)=\mu^{2} x$.

(iv) $\left( \pm \omega_{1}, 0, \pm \omega_{3}, \sqrt{I_{1}-I_{3}}, 0\right)$, eixo $z^{\prime}$. Onde $\omega_{3}^{2}=k\left(1-x_{0}\left(\sqrt{I_{1}-I_{3}}\right)^{-1}\right)$.

$\mathrm{E}\left(I_{2}+I_{1}-I_{3}\right)^{2} \omega_{2}^{2}=\mu^{2}-k I_{1}^{2}\left(1-x_{0}\left(\sqrt{I_{1}-I_{3}}\right)^{-1}\right)$.

(v) $\left( \pm \omega_{1}, 0, \mp \omega_{3}, \sqrt{I_{1}-I_{3}}, 0\right)$, eixo $z^{\prime \prime}$. Onde $\omega_{3}^{2}=k\left(1-x_{0}\left(\sqrt{I_{1}-I_{3}}\right)^{-1}\right)$.

$\mathrm{E}\left(I_{2}+I_{1}-I_{3}\right)^{2} \omega_{2}^{2}=\mu^{2}-k I_{1}^{2}\left(1-x_{0}\left(\sqrt{I_{1}-I_{3}}\right)^{-1}\right)$. 
(vi) $\left( \pm \omega_{1}, \pm \omega_{2}, 0, \sqrt{I_{1}-I_{2}}, 0\right)$, eixo $y^{\prime}$. Onde $\omega_{2}^{2}=k\left(1-x_{0}\left(\sqrt{I_{1}-I_{2}}\right)^{-1}\right)$.

$\mathrm{E}\left(I_{3}+I_{1}-I_{2}\right)^{2} \omega_{2}^{2}=\mu^{2}-k I_{1}^{2}\left(1-x_{0}\left(\sqrt{I_{1}-I_{2}}\right)^{-1}\right)$.

(vii) $\left( \pm \omega_{1}, \mp \omega_{2}, 0, \sqrt{I_{1}-I_{2}}, 0\right)$, eixo $y^{\prime \prime}$. Onde $\omega_{2}^{2}=k\left(1-x_{0}\left(\sqrt{I_{1}-I_{2}}\right)^{-1}\right)$.

$\mathrm{E}\left(I_{3}+I_{1}-I_{2}\right)^{2} \omega_{2}^{2}=\mu^{2}-k I_{1}^{2}\left(1-x_{0}\left(\sqrt{I_{1}-I_{2}}\right)^{-1}\right)$.

Ou seja, existem no máximo os sete eixos de equilíbrio acima. De tal forma que os quatro últimos desaparecem exatamente para os valores de parâmetros enunciados. Ou seja, ocorrem bifurcações (pelo menos) nestes valores de parâmetro.

A análise detalhada destas afirmações é simples, porém tediosa. Por isso é omitida.

Observe que para os mesmos quatro últimos equilíbrios listados, a posição de equilíbrio das massas é independente da constante de elasticidade da mola.

Além disso, nestes casos o elipsóide (efetivo) de inércia possui simetria com respeito aos eixos $y$ ou $z$. 


\section{Capítulo 3}

\section{Equações de movimento}

A discussão desse capítulo é sobre a evolução temporal de um sistema mecânico (contínuo) que não seja um corpo rígido ideal, mas que contenha componentes rígidas e elásticas ou que seja deformável. Contanto que esta deformação não seja expressiva ao ponto de não ser possível definir um referencial natural (não inercial) que acompanhe o mesmo.

O tratamento é o desenvolvido na referência [BL87].

Faz-se necessário estabelecer a notação e os conceitos relacionados à dinâmica do corpo rígido ideal. Será trabalhada aqui uma formulação Lagrangiana para este tipo de problema.

Uma referência suficiente para este assunto se encontra em [Arn89].

\subsection{Conceitos preliminares}

A classe de sistemas mecânicos do tipo corpo rígido consiste naqueles que possuem como vínculos (holonômicos):

$$
\left\|r\left(x_{1}, t\right)-r\left(x_{2}, t\right)\right\|=r_{12}, \quad \forall t \geq 0 .
$$

Onde $x_{1}, x_{2}$ são coordenadas de pontos do corpo na posição inicial, e $r\left(x_{1}, t\right), r\left(x_{2}, t\right)$ são suas posições após um tempo $t$ ( $r_{12}$ é constante no tempo).

Como é conhecido, da formulação Lagrangiana da mecânica clássica, a descrição do sistema é completamente determinada por um conjunto de coordenadas associadas aos graus de liberdade do mesmo.

No caso do corpo rígido, os graus de liberdade são determinados pelos vínculos já citados. $\mathrm{E}$ as coordenadas (independentes) que completamente o descrevem são $(Y, y) \in S O(3) \times \mathbb{R}^{3}$.

As coordenadas $Y, y$ representam, respectivamente, a rotação de um referencial fixo ao corpo e o deslocamento de sua origem (o qual move-se "rigidamente" com o corpo), em relação ao referencial inercial adotado.

O método proposto para descrever um corpo "aproximadamente rígido", como mencionado no início, é adicionar às coordenadas acima uma coordenada "contínua", $u$, associada às deformações ou oscilações relativas das componentes do corpo, no referencial móvel.

O espaço ao qual pertence esta $u$ não será estabelecido aqui. É conveniente assumir que $u(\cdot, t) \in$ $\mathcal{C}\left(\mathcal{B}, \mathbb{R}^{3}\right)$, que em geral está contido em um espaço vetorial $\left(\mathcal{B} \in \mathbb{R}^{3}\right.$ delimita a extensão espacial do corpo na posição inicial, ou relaxada, isto é, sem deformações, e $\mathcal{C}\left(\mathcal{B}, \mathbb{R}^{3}\right)$ um espaço de funções contínuas de $\mathcal{B}$ em $\left.\mathbb{R}^{3}\right)$. A descrição deste espaço será feita de acordo com a necessidade do problema.

As coordenadas $(Y, y)$ determinam um referencial no qual o corpo está em repouso. Qualquer vetor $v$ nesse referencial é representado no referencial inercial como $V$ através da equação:

$$
V(t)=Y(t) v(t)+y(t)
$$


É um resultado básico do tratamento do corpo rígido que $\forall t \geq 0, \dot{Y}(t)=Y(t) \Omega(t)$. Onde,

$$
\Omega(t)=\left(\begin{array}{ccc}
0 & -\omega_{3} & \omega_{2} \\
\omega_{3} & 0 & -\omega_{1} \\
-\omega_{2} & \omega_{1} & 0
\end{array}\right)
$$

Sendo $\omega(t)=\left(\omega_{1}, \omega_{2}, \omega_{3}\right)$ a velocidade angular do corpo descrita no referencial móvel. Vale ressaltar que em [Arn89], prova-se que $\dot{Y}=\Omega Y$, mas neste contexto $\Omega$ está descrito no referencial inercial, o que causa a troca na ordem.

Assim, sendo $U: \mathcal{B} \times \mathbb{R} \longrightarrow \mathbb{R}^{3}$, onde $U(z, t)$ é a posição do ponto $z$ do corpo (não deformado), no referencial inercial, no instante $t$, então por (3.1), temos:

$$
U_{t}=Y \Omega u+Y u_{t}+\dot{y}=Y\left(\Omega u+u_{t}\right)+\dot{y} .
$$

Portanto, a energia cinética do sistema é

$$
T=\frac{1}{2} \int_{\mathcal{B}}\left\|U_{t}(z, t)\right\|^{2} d m=\frac{1}{2} \int_{\mathcal{B}}\left\|\Omega(t) u(z, t)+u_{t}(z, t)+Y(t)^{-1} \dot{y}(t)\right\|^{2} d m
$$

Onde $d m$ é a medida de massa do corpo no instante inicial (posição relaxada) e $Y(t) \in S O(3) \Rightarrow$ $\|Y v\|=\|v\|, \forall v \in \mathbb{R}^{3}$.

\subsection{Dedução das equações}

Para deduzir as equações de movimento, observe que uma das coordenadas que o descrevem está sobre um grupo de Lie, $S O(3)$ (com a multiplicação usual de matrizes). Assim, é conveniente estudar a diferenciação de funções sobre este espaço.

A discussão que segue objetiva deduzir a equação (3.6) (a ser apresentada). Ela é apresentada em um contexto menos concreto, porém, sem maiores dificuldades do que o caso de interesse.

Seja $M$ uma variedade diferenciável de dimensão $n$.

Associado a $M$, temos seu fibrado tangente, $T M:=\bigcup_{x \in M}\{x\} \times T_{x} M$. Este possui uma estrutura de variedade diferenciável, dada pelo atlas: $\mathcal{A}_{T M}=\{d \varphi: \varphi \in \mathcal{A}\}$. Onde, $\mathcal{A}$ é o atlas de $M$. Se $\varphi: U \subset M \longrightarrow \tilde{U} \subset \mathbb{R}^{n}$, então $d \varphi: T U \longrightarrow \tilde{U} \times \mathbb{R}^{n}, d \varphi(x, v):=\left(\varphi(x), d \varphi_{x} v\right)$, sendo $T U=\bigcup_{x \in U}\{x\} \times T_{x} M$.

Assim, dado $x \in M$, existe carta $\varphi: U \subset M \longrightarrow \tilde{U} \subset \mathbb{R}^{n}$ tal que $x \in U$. Logo, $d \varphi$ é um difeomorfismo, tendo $\{x\} \times T_{x} M$ como um aberto de seu domínio.

Seja $L: T M \longrightarrow \mathbb{R}$ uma função de classe $C^{\infty}$. Tome $x \in M$ e uma carta $\varphi$ tal que $x$ esteja em seu domínio. Como foi discutido acima, $\mathcal{L}:=L \circ d \varphi^{-1}: \tilde{U} \times \mathbb{R}^{n} \longrightarrow \mathbb{R}$ é de classe $C^{\infty}$.

Definição 3.2.1. Uma função de classe $C^{\infty}, \gamma: I \subset \mathbb{R} \longrightarrow M$, com $0 \in I, \gamma(0)=x$ (I intervalo aberto), satisfaz à equaçẫo de Euler-Lagrange em $x$ (em relação a L) se existe $\varphi: x \in U \longrightarrow \tilde{U} \subset$ $\mathbb{R}^{n}$ carta de $M$ tal que

$$
\frac{d}{d t}\left(\frac{\partial \mathcal{L}}{\partial \dot{Q}}\left(d \varphi\left(\gamma(t), \gamma^{\prime}(t)\right)\right)\right)-\frac{\partial \mathcal{L}}{\partial Q}\left(d \varphi\left(\gamma(t), \gamma^{\prime}(t)\right)\right)=0, \quad \forall t \in \gamma^{-1}(\gamma(I) \cap U)
$$

Onde, $\mathcal{L}$ é obtida de $L$ como anteriormente através de $\varphi$, notando que, como foi definida, $\mathcal{L} \in$ $\mathcal{C}^{\infty}\left(\tilde{U} \times \mathbb{R}^{n}\right)$. Assim, como usual, se $(\alpha(t), \beta(t)) \in \tilde{U} \times \mathbb{R}^{n}$,

$$
\frac{\partial \mathcal{L}}{\partial \dot{Q}}(\alpha(t), \beta(t)):=d(\mathcal{L}(\alpha(t), \cdot))_{\beta(t)} \in\left(\mathbb{R}^{n}\right)^{*} \quad \Longrightarrow \frac{d}{d t}\left(\frac{\partial \mathcal{L}}{\partial \dot{Q}}(\alpha(t), \beta(t))\right) \in\left(\mathbb{R}^{n}\right)^{*}, \quad \forall t .
$$


Analogamente,

$$
\frac{\partial \mathcal{L}}{\partial Q}(\alpha(t), \beta(t)):=d(\mathcal{L}(\cdot, \beta(t)))_{\alpha(t)} \in\left(\mathbb{R}^{n}\right)^{*}
$$

Portanto, a equação estabelece que, para cada $t$ no domínio descrito, este funcional (sobre $\mathbb{R}^{n}$ ) é identicamente nulo.

Um resultado que segue diretamente da equação (1.1) (tomando $\mathcal{D} \equiv 0$ ) é que a definição anterior não depende da carta. Ou seja, se $\gamma$ satisfaz à equação (3.3), então, para qualquer $\psi: x \in$ $V \subset M \longrightarrow \tilde{V}$

$$
\frac{d}{d t}\left(\frac{\partial \hat{\mathcal{L}}}{\partial \dot{Q}}\left(d \psi\left(\gamma(t), \gamma^{\prime}(t)\right)\right)\right)-\frac{\partial \hat{\mathcal{L}}}{\partial Q}\left(d \psi\left(\gamma(t), \gamma^{\prime}(t)\right)\right)=0, \quad \forall t \in \gamma^{-1}(\gamma(I) \cap U \cap V)
$$

$\hat{\mathcal{L}}:=L \circ d \psi^{-1}$.

Teorema 3.2.1. Seja $M$ uma variedade diferenciável e $M_{0} \subset M$ uma subvariedade de dimensão $k \leq n$. Suponha que $L \in \mathcal{C}^{\infty}(T M)$. Sendo assim, se $\gamma: I \longrightarrow M_{0}$ satisfaz à equação de EulerLagrange em $x \in M_{0}$ (em relação a $\left.L\right|_{T M_{0}}$ ), então, existe $\varphi: x \in U \subset M \longrightarrow \tilde{U}$, carta, tal que

$$
\left\{\frac{d}{d t}\left(\frac{\partial \mathcal{L}}{\partial \dot{Q}}\left(d \varphi\left(\gamma(t), \gamma^{\prime}(t)\right)\right)\right)-\frac{\partial \mathcal{L}}{\partial Q}\left(d \varphi\left(\gamma(t), \gamma^{\prime}(t)\right)\right)\right\}(0, v)=0 \quad \forall v \in \mathbb{R}^{k}, \forall t .
$$

Onde, $(0, v) \in \mathbb{R}^{n-k} \times \mathbb{R}^{k} \equiv \mathbb{R}^{n}$ é um vetor cujas $n-k$ primeiras componentes são nulas, e as demais são as componentes de $v$.

Demonstração. Como $M_{0}$ é subvariedade de $M$, existe uma carta de subvariedade de $M_{0}$ em $x$. Isto é, existe $\varphi$, como mencionada no enunciado, e tal que $\varphi\left(U \cap M_{0}\right)=\{0\}_{n-k} \times \tilde{U}_{0} \subset\{0\}_{n-k} \times \mathbb{R}^{k}$, onde $\operatorname{dim} M=n \geq \operatorname{dim} M_{0}=k$.

Para facilitar a prova, defina, $P_{2}: \mathbb{R}^{n} \longrightarrow \mathbb{R}^{k}$ e $J_{2}: \mathbb{R}^{k} \longrightarrow \mathbb{R}^{n}$, tais que:

$$
P_{2}(a, b):=b, \quad J_{2}(b):=(0, b) .
$$

Defina também a inclusão, $i: M_{0} \longrightarrow M, i(x)=x, \forall x \in M_{0}$.

Sendo assim, é fácil verificar que $\varphi_{0}:=P_{2} \circ \varphi \circ i: U \cap M_{0} \longrightarrow \tilde{U}_{0}$, é carta de $M_{0}$. Além disso, $\forall y \in U \cap M_{0}, \varphi \circ i(y)=(0, u) \in\{0\}_{n-k} \times \tilde{U}_{0}$. Mas, $\varphi_{0}(y)=P_{2}(0, u)=u \Rightarrow \varphi \circ i=J_{2} \circ \varphi_{0}$.

Logo, pela hipótese,

$$
\frac{d}{d t}\left(\frac{\partial \mathcal{L}_{0}}{\partial \dot{Q}}\left(d \varphi_{0}\left(\gamma(t), \gamma^{\prime}(t)\right)\right)\right)-\frac{\partial \mathcal{L}_{0}}{\partial Q}\left(d \varphi_{0}\left(\gamma(t), \gamma^{\prime}(t)\right)\right)=0
$$

Onde, $\mathcal{L}_{0}:=\left.L\right|_{T M_{0}} \circ d \varphi_{0}^{-1}=L \circ d i \circ d \varphi_{0}^{-1}=L \circ d\left(i \circ \varphi_{0}^{-1}\right)=L \circ d\left(\varphi^{-1} \circ J_{2}\right)=\mathcal{L} \circ J_{2}$. Sendo $\mathcal{L}:=L \circ d \varphi^{-1}$.

Desta forma,

$$
\begin{gathered}
\left.\left.\frac{\partial \mathcal{L}}{\partial \dot{Q}}\left(d \varphi\left(\gamma(t), \gamma^{\prime}(t)\right)\right) \circ J_{2}=\frac{\partial \mathcal{L}}{\partial \dot{Q}}\left(J_{2} \circ \varphi_{0}(\gamma(t)), J_{2} \circ d\left(\varphi_{0}\right)_{\gamma(t)} \gamma^{\prime}(t)\right)\right) \circ J_{2}=\frac{\partial \mathcal{L} \circ J_{2}}{\partial \dot{Q}}\left(\varphi_{0}(\gamma(t)), d\left(\varphi_{0}\right)_{\gamma(t)} \gamma^{\prime}(t)\right)\right) \\
=\frac{\partial \mathcal{L}_{0}}{\partial \dot{Q}}\left(d \varphi_{0}\left(\gamma(t), \gamma^{\prime}(t)\right)\right) .
\end{gathered}
$$

Assim como,

$$
\begin{gathered}
\left.\left.\frac{\partial \mathcal{L}}{\partial Q}\left(d \varphi\left(\gamma(t), \gamma^{\prime}(t)\right)\right) \circ J_{2}=\frac{\partial \mathcal{L}}{\partial Q}\left(J_{2} \circ \varphi_{0}(\gamma(t)), J_{2} \circ d\left(\varphi_{0}\right)_{\gamma(t)} \gamma^{\prime}(t)\right)\right) \circ J_{2}=\frac{\partial \mathcal{L} \circ J_{2}}{\partial Q}\left(\varphi_{0}(\gamma(t)), d\left(\varphi_{0}\right)_{\gamma(t)} \gamma^{\prime}(t)\right)\right) \\
=\frac{\partial \mathcal{L}_{0}}{\partial Q}\left(d \varphi_{0}\left(\gamma(t), \gamma^{\prime}(t)\right)\right)
\end{gathered}
$$


Segue que, de acordo com (3.4),

$$
\begin{gathered}
\frac{d}{d t}\left(\frac{\partial \mathcal{L}_{0}}{\partial \dot{Q}}\left(d \varphi_{0}\left(\gamma(t), \gamma^{\prime}(t)\right)\right)\right)-\frac{\partial \mathcal{L}_{0}}{\partial Q}\left(d \varphi_{0}\left(\gamma(t), \gamma^{\prime}(t)\right)\right)= \\
=\left(\frac{d}{d t}\left(\frac{\partial \mathcal{L}}{\partial \dot{Q}}\left(d \varphi\left(\gamma(t), \gamma^{\prime}(t)\right)\right)\right)-\frac{\partial \mathcal{L}}{\partial Q}(d \varphi(\gamma(t), \gamma(t)))\right) \circ J_{2} \equiv 0
\end{gathered}
$$

Caso $M$ seja uma variedade riemanniana, usando a equação (3.5) e a relação $J_{2} \circ d\left(\varphi_{0}\right)_{\gamma(t)}=$ $d\left(J_{2} \circ \varphi_{0}\right)_{\gamma(t)}=d \varphi_{\gamma(t)} \circ d i_{\gamma(t)}$, obtemos que:

$$
\left[\left(\frac{d}{d t}\left(\frac{\partial \mathcal{L}}{\partial \dot{Q}}\left(\tilde{\varphi}\left(\gamma(t),(i \circ \gamma)^{\prime}(t)\right)\right)\right)-\frac{\partial \mathcal{L}}{\partial Q}\left(\tilde{\varphi}\left(\gamma(t),(i \circ \gamma)^{\prime}(t)\right)\right)\right) \circ d \varphi_{\gamma(t)}\right] \circ d i_{\gamma(t)}=0
$$

Novamente, pela equação (1.1), esta equação vale para toda carta. Ou seja, este funcional, que pertence a $T_{\gamma(t)} M^{*}$, tem restrição nula ao subespaço de $T_{\gamma(t)} M$, (identificado pela aplicação injetora $d i_{\gamma(t)}$ a) $T_{\gamma(t)} M_{0}$.

Devido à existência da métrica riemanniana, para cada $t$ no domínio adequado existe um único vetor em $T_{\gamma(t)} M$ que representa este funcional (com relação à métrica, da forma usual).

Este vetor será representado simplesmente, sem fazer menção à carta (já que é o mesmo para todas), por:

$$
\frac{d}{d t}\left(\frac{\partial \mathcal{L}}{\partial \dot{Q}}\left(\gamma(t), \gamma^{\prime}(t)\right)\right)-\frac{\partial \mathcal{L}}{\partial Q}\left(\gamma(t), \gamma^{\prime}(t)\right) \in T_{\gamma(t)} M_{0}^{\perp}
$$

Portanto, a equação (3.5) é equivalente a:

$$
\pi_{\gamma(t)}\left(\frac{d}{d t}\left(\frac{\partial \mathcal{L}}{\partial \dot{Q}}\left(\gamma(t), \gamma^{\prime}(t)\right)\right)-\frac{\partial \mathcal{L}}{\partial Q}\left(\gamma(t), \gamma^{\prime}(t)\right)\right)=0 \quad \forall t .
$$

Onde $\pi_{q}: T_{q} M \longrightarrow T_{q} M_{0}$ é a projeção ortogonal, $\forall q \in M_{0}$.

No contexto deste trabalho, a variedade de interesse que é determinada pelas restrições do movimento do sistema físico é $S O(3) \times \mathbb{R}^{3} \times \mathcal{C}$. Observe que, desde que $S O(3) \subset G L(3)$ é uma subvariedade, $S O(3) \times \mathbb{R}^{3} \times \mathcal{C}$ é subvariedade de $G L(3) \times \mathbb{R}^{3} \times \mathcal{C}$. Note que podemos munir esta última (e portanto a primeira também) com uma métrica natural, pois cada uma das variedades no produto possui a sua, quais sejam:

$$
\langle X, Y\rangle_{G L(3)}:=\operatorname{Tr}\left(X^{t} Y\right) \quad \forall X, Y \in M(3), \quad\langle x, y\rangle_{\mathbb{R}^{3}}=x . y, \quad\langle u, w\rangle_{\mathcal{C}}:=\int_{B} u(x) w(x) d x .
$$

Lembrando que $T_{A} G L(3)=M(3), \forall A \in G L(3)$.

Observação 3.2.1. Como mencionado na seção anterior, a especificação do espaço de funções $\mathcal{C}$ é feita para cada modelo. Nas principais referências usadas neste trabalho, [BL87] e [BL91], não foram definidas com detalhes as propriedades de seus elementos.

No presente trabalho, este espaço será tratado de maneira informal. Foi chamado de variedade diferenciável, mas não haverá uma discussão mais séria sobre isto. Com base nas referências, pode-se sugerir pensar em $\mathcal{C}$ como um conjunto de difeomorfismos, "contendo a identidade em seu interior".

$O$ tratamento dado aos espaços $S O(3)$ e $\mathbb{R}^{3}$ tem fundamentos. Este tratamento será aplicado também para o espaço de funçôes, tentando seguir as ideias mais elementares de cálculo de variações. Tendo como "espaço tangente" em um ponto $u \in \mathcal{C}$, o conjunto das funçôes $h: \mathcal{B} \longrightarrow \mathbb{R}^{3}$ tais que exista uma familia suave $\phi_{\epsilon}$ em $\mathcal{C}$ com $\phi_{0}=u$ e $\left.\partial_{\epsilon} \phi_{\epsilon}\right|_{\epsilon=0}=h$.

Assim, fica definida a métrica em $G L(3) \times \mathbb{R}^{3} \times \mathcal{C}$ :

$$
\left\langle\left(X, x, h_{1}\right),\left(Y, y, h_{2}\right)\right\rangle_{G L(3) \times \mathbb{R}^{3} \times \mathcal{C}}:=\operatorname{Tr}\left(X^{T} Y\right)+x . y+\int_{B} h_{1}(x) h_{2}(x) d x .
$$


A seguir será suposto, como princípio, que a evolução temporal do sistema é dada pelas soluções das equações de Euler-Lagrange.

No caso de interesse, o sistema mecânico consiste em um corpo (contínuo) que pode sofrer pequenas deformações, dissipando energia, sendo que a Lagrangiana do sistema tem a forma: $L$ : $T\left(S O(3) \times \mathbb{R}^{3} \times \mathcal{C}\right) \cong T S O(3) \times \mathbb{R}^{6} \times(\mathcal{C} \times \mathcal{V}) \longrightarrow \mathbb{R}, \mathrm{com}$

$$
L\left(Y, y, u, \dot{Y}, \dot{y}, u_{t}\right)=T\left(Y, y, u, \dot{Y}, \dot{y}, u_{t}\right)-V(Y, y, u)
$$

Onde $T$ é dado pela equação (3.2) e $V$ depende das características (digamos "internas") do corpo em consideração, sendo suposto que $V$ é constante relativamente às variáveis $\dot{Y}, \dot{y}, u_{t}$. Esta é uma suposição que é feita em geral. A dependência sobre as velocidades é deixada para uma "função de dissipação".

Assim, dado $(Y, y, u) \in S O(3) \times \mathbb{R}^{3} \times \mathcal{C}$ e recordando que esta variedade é subvariedade de um (aberto de um) espaço vetorial, $M(3) \times \mathbb{R}^{3} \times \mathcal{C}$, o funcional de Euler-Lagrange associado a $L$ pode ser encontrado através do Teorema 3.2.1.

Observação 3.2.2. SO(3) possui a estrutura de grupo de Lie, com a multiplicação usual de matrizes. Assim, cada espaço tangente é facilmente calculado: $T_{Y} S O(3)=Y T_{I} S O(3)=Y$ Ant(3), onde Ant(3) é o conjunto das matrizes $3 \times 3$ antissimétricas.

Para isto, observe a seguinte propriedade da projeção ortogonal:

$\pi_{(Y, y, u)}=\left(P_{Y} \times I d_{\mathbb{R}^{3}} \times I d_{\mathcal{V}}\right): M(3) \times \mathbb{R}^{3} \times \mathcal{V} \longrightarrow T_{Y} S O(3) \times \mathbb{R}^{3} \times \mathcal{V}$

Onde $P_{Y}$ é facilmente calculada no próximo lema.

Lema 3.2.1. Para todo $Y \in S O(3)$ e todo $A \in M(3)$,

$$
P_{Y} A=Y\left(Y^{T} A\right)_{a}
$$

Onde $B_{a}:=1 / 2\left(B-B^{T}\right)$ é a parte antissimétrica de uma matriz $B$.

Demonstração. Sabe-se que $T_{I} G L(3)=M(3)=\operatorname{Sym}(3) \oplus \operatorname{Ant}(3)$ (e ainda que $\left.\operatorname{Sym}(3) \perp \operatorname{Ant}(3)\right)$. E portanto, $M(3)=Y M(3)=T_{Y} G L(3)=Y \operatorname{Sym}(3) \oplus Y \operatorname{Ant}(3)=Y \operatorname{Sym}(3) \oplus T_{Y} S O(3)$. (De fato, se $B \in Y \operatorname{Sym}(3) \cap Y \operatorname{Ant}(3)$, existem $C_{1} \in \operatorname{Sym}(3), C_{2} \in \operatorname{Ant}(3)$ tais que $Y C_{1}=B=Y C_{2}$, logo $\left.C_{1} Y^{T}=\left(Y C_{1}\right)^{T}=\left(Y C_{2}\right)^{T}=-C_{2} Y^{T} \Rightarrow C_{1}=-C_{2} \Rightarrow B=0\right)$.

Além disso, como $Y \operatorname{Sym}(3) \perp Y \operatorname{Ant}(3)$, e

$$
A=Y\left(Y^{T} A\right)=Y \frac{\left(Y^{T} A+\left(Y^{T} A\right)^{T}\right)}{2}+Y \frac{\left(Y^{T} A-\left(Y^{T} A\right)^{T}\right)}{2} \in Y \operatorname{Sym}(3) \oplus Y \operatorname{Ant}(3),
$$

temos que:

$$
P_{Y} A=Y \frac{\left(Y^{T} A-\left(Y^{T} A\right)^{T}\right)}{2}=Y\left(Y^{T} A\right)_{a}
$$

Também será necessário o seguinte lema.

Lema 3.2.2. Seja $T: T G L(3) \longrightarrow \mathbb{R}$ uma função invariante à esquerda, ou seja, tal que exista $K: M(3) \longrightarrow \mathbb{R}$, e $T(Y, \dot{Y})=K\left(Y^{-1} \dot{Y}\right)$, para todo $(Y, \dot{Y}) \in T G L(3)$. Assim, para toda curva $C^{\infty}$, $Y(t) \in S O(3),(Y, \dot{Y}) \in T S O(3)$,

$$
P_{Y}\left(\frac{d}{d t}\left(\frac{\partial T}{\partial \dot{Y}}\right)-\frac{\partial T}{\partial Y}\right)=Y\left(\frac{d A}{d t}(t)+[\Omega, A(t)]\right)
$$

Onde $\Omega=Y^{T} \dot{Y}$ e $A(t)=\left(\frac{\partial K}{\partial \Omega}\right)_{a}$ 
Demonstração. $T G L(3)=G L(3) \times M(3)$ é um aberto do espaço vetorial $M(3) \times M(3)$. Portanto, de forma consistente com a discussão anterior,

$$
\frac{\partial T}{\partial \dot{Y}}(Y, \dot{Y}) \cong d_{\dot{Y}}(T(Y, .)) \in M(3)^{*}, \quad \frac{\partial T}{\partial Y}(Y, \dot{Y}) \cong d_{Y}(T(., \dot{Y})) \in M(3)^{*} .
$$

Onde a identificação é dada pela métrica em $G L(3)$.

Considere a função $\Omega: G L(3) \times M(3) \longrightarrow M(3), \Omega\left(B_{1}, B_{2}\right):=B_{1}^{-1} B_{2}$.

É claro que $\Omega\left(B_{1},.\right)$ é linear. Portanto, $d_{\dot{Y}} \Omega(Y,) B=.Y^{-1} B$.

Temos também que, $\forall H \in G L(3)-\{0\}$, pequeno o suficiente para que $Y+H \in G L(3)$,

$$
\begin{gathered}
\frac{\Omega(Y+H, \dot{Y})-\Omega(Y, \dot{Y})+Y^{-1} H Y^{-1} \dot{Y}}{\|H\|}=\frac{\left((Y+H)^{-1}-Y^{-1}+Y^{-1} H Y^{-1}\right) \dot{Y}}{\|H\|}= \\
=\frac{(Y+H)^{-1}\left(I d-(Y+H) Y^{-1}+(Y+H) Y^{-1} H Y^{-1}\right) \dot{Y}}{\|H\|}= \\
=\frac{(Y+H)^{-1}\left(-H Y^{-1}+H Y^{-1}+H Y^{-1} H Y^{-1}\right) \dot{Y}}{\|H\|}=(Y+H)^{-1} \frac{H Y^{-1} H Y^{-1}}{\|H\|} \dot{Y} .
\end{gathered}
$$

Logo,

$$
\frac{\left\|\Omega(Y+H, \dot{Y})-\Omega(Y, \dot{Y})+Y^{-1} H Y^{-1} \dot{Y}\right\|}{\|H\|} \leq\left\|(Y+H)^{-1}\right\| \frac{\|H\|^{2}\left\|Y^{-1}\right\|^{2}}{\|H\|}\|\dot{Y}\| \stackrel{H \rightarrow 0}{\longrightarrow} 0 .
$$

Pois

$$
\lim _{H \rightarrow 0}(Y+H)^{-1}=Y^{-1} .
$$

Logo, $d_{Y} \Omega(., \dot{Y}) B=-Y^{-1} B Y^{-1} \dot{Y}, \forall B \in M(3)$.

Assim, $\forall B \in M(3)$,

$$
\begin{gathered}
d_{Y}(T(., \dot{Y}))(B)=d_{\Omega} K(\Omega)\left(d_{Y}(\Omega(., \dot{Y})) B\right)=-d_{\Omega} K(\Omega)\left(Y^{-1} B Y^{-1} \dot{Y}\right) \\
d_{\dot{Y}}(T(Y, .))(B)=d_{\Omega} K(\Omega)\left(d_{\dot{Y}}(\Omega(Y, .)) B\right)=d_{\Omega} K(\Omega)\left(Y^{-1} B\right)
\end{gathered}
$$

Usando novamente a identificação pela métrica, e o fato que $Y^{-1}=Y^{T}$, as equações anteriores fornecem que, $\forall B \in M(3)$ :

$$
\begin{gathered}
\left\langle\frac{\partial T}{\partial Y}, B\right\rangle=-\left\langle\frac{\partial K}{\partial \Omega}, Y^{-1} B Y^{-1} \dot{Y}\right\rangle=-\operatorname{Tr}\left(\left(\frac{\partial K}{\partial \Omega}\right)^{T} Y^{T} B Y^{T} \dot{Y}\right)=-\operatorname{Tr}\left(\left(Y \frac{\partial K}{\partial \Omega}\right)^{T} B Y^{T} \dot{Y}\right)= \\
=-\operatorname{Tr}\left(\left(Y^{T} \dot{Y}\right)\left(Y \frac{\partial K}{\partial \Omega}\right)^{T} B\right)=-\operatorname{Tr}\left(\left(Y \frac{\partial K}{\partial \Omega} \Omega^{T}\right)^{T} B\right)=\left\langle-Y \frac{\partial K}{\partial \Omega} \Omega^{T}, B\right\rangle \\
\left\langle\frac{\partial T}{\partial \dot{Y}}, B\right\rangle=\left\langle\frac{\partial K}{\partial \Omega}, Y^{-1} B\right\rangle=\operatorname{Tr}\left(\left(Y \frac{\partial K}{\partial \Omega}\right)^{T} B\right)=\left\langle Y \frac{\partial K}{\partial \Omega}, B\right\rangle .
\end{gathered}
$$

Portanto,

$$
\frac{d}{d t}\left(\frac{\partial T}{\partial \dot{Y}}\right)-\frac{\partial T}{\partial Y}=\frac{d}{d t}\left(Y \frac{\partial K}{\partial \Omega}\right)+Y \frac{\partial K}{\partial \Omega} \Omega^{T}=Y \frac{d}{d t}\left(\frac{\partial K}{\partial \Omega}\right)+\dot{Y} \frac{\partial K}{\partial \Omega}+Y \frac{\partial K}{\partial \Omega} \Omega^{T} .
$$

Logo,

$$
P_{Y}\left(\frac{d}{d t}\left(\frac{\partial T}{\partial \dot{Y}}\right)-\frac{\partial T}{\partial Y}\right)=Y\left(\frac{d}{d t}\left(\frac{\partial K}{\partial \Omega}\right)+Y^{T} \dot{Y} \frac{\partial K}{\partial \Omega}+\frac{\partial K}{\partial \Omega} \Omega^{T}\right)_{a}=
$$




$$
=Y\left(\frac{d A}{d t}+\frac{1}{2}\left(\Omega \frac{\partial K}{\partial \Omega}+\frac{\partial K}{\partial \Omega} \Omega^{T}-\left(\frac{\partial K}{\partial \Omega}\right)^{T} \Omega^{T}-\Omega\left(\frac{\partial K}{\partial \Omega}\right)^{T}\right)\right)=Y\left(\frac{d A}{d t}+\Omega A+A \Omega^{T}\right) .
$$

Se recordarmos que $\dot{Y} \in T_{Y} S O(3)=Y \operatorname{Ant}(3)$, veremos que existe uma matriz antissimétrica $M$ tal que $\dot{Y}=Y M$. Logo, $\Omega=Y^{T} \dot{Y}=M$, é antissimétrica. Assim, segue o resultado do lema.

Observação 3.2.3. Podemos fazer uma identificação natural $M(3) \equiv \mathbb{R}^{9}$ agrupando as colunas de cada matriz em sequência (transpostas) formando um vetor de nove entradas. É fácil ver que esta identificação (isomorfismo linear) preserva os produtos internos em questão. Portanto, o vetor $\partial K / \partial \Omega$ que representa o funcional $d_{\Omega} K$ é a derivada usual de $K$.

Retornando à Lagrangiana de interesse, descrita por (3.7), temos que a equação de EulerLagrange associada a ela, e à curva $\gamma(t)=(Y(t), y(t), u(t))$, é:

$$
\pi_{(Y, y, u)}\left(\frac{d}{d t}\left(\frac{\partial \mathcal{L}}{\partial \dot{Q}}\left(\gamma(t), \gamma^{\prime}(t)\right)\right)-\frac{\partial \mathcal{L}}{\partial Q}\left(\gamma(t), \gamma^{\prime}(t)\right)\right)=0 .
$$

Ou, explicitamente,

$$
\begin{gathered}
P_{Y}\left(\frac{d}{d t}\left(\frac{\partial L}{\partial \dot{Y}}\right)-\frac{\partial L}{\partial Y}\right)=0 \\
\frac{d}{d t}\left(\frac{\delta L}{\delta v}\right)-\frac{\delta L}{\delta u}=0 \\
\frac{d}{d t}\left(\frac{\partial L}{\partial \dot{y}}\right)-\frac{\partial L}{\partial y}=0
\end{gathered}
$$

A equação (3.13) necessita de esclarecimento. Novamente, não será detalhada e estrutura do espaço de funções. Alguma restrição desnecessária que fosse imposta a ele poderia limitar a aplicabilidade do resultado apresentado.

O símbolo $\delta$ é usado sem significado preciso. Neste trabalho ele terá o seguinte sentido:

Dados $F: \mathcal{C} \longrightarrow \mathbb{R}, u \in \mathcal{C}$ e $h \in \mathcal{V}:=T_{u} \mathcal{C}$, defina:

$$
\delta_{u} F(h):=\left.\frac{d}{d \epsilon} F\left(\phi_{\epsilon}\right)\right|_{\epsilon=0} .
$$

Caso exista a derivada. Onde $\phi_{0}=u$ e $d \phi_{\epsilon} /\left.d \epsilon\right|_{\epsilon=0}=h$ Este funcional, claramente linear, é usualmente chamado derivada direcional de $F$.

Chamaremos de $\delta F / \delta u \in \mathcal{V}$ a função (única, se existir) tal que

$$
\delta_{u} F(h)=\int_{B}\left\langle h, \frac{\delta F}{\delta u}\right\rangle d x, \forall h \in \mathcal{V} .
$$

Ainda na mesma equação, o símbolo $\delta L / \delta v$ denota a derivada com respeito à variável $v:=u_{t}$, da mesma maneira.

Este sistema de equações não atende completamente às necessidades do modelo o qual este trabalho se propõe tratar. O sistema completo será estabelecido adicionando um termo específico em cada equação. Essa estrutura momentânea servirá apenas para facilitar o desenvolvimento de cada termo.

A equação (3.12) é equivalente a:

$$
P_{Y}\left(\frac{d}{d t}\left(\frac{\partial T}{\partial \dot{Y}}\right)-\frac{\partial T}{\partial Y}\right)=-P_{Y}\left(\frac{\partial V}{\partial Y}\right) .
$$


Além disso,

$$
T=T_{1}+T_{2}+T_{3}=\frac{1}{2} \int_{\mathcal{B}}\left\|\Omega u+u_{t}\right\|^{2} d m+\int_{\mathcal{B}}\left\langle\Omega u+u_{t}, Y^{-1} \dot{y}\right\rangle d m+\frac{1}{2} \int_{\mathcal{B}}\|\dot{y}\|^{2} d m .
$$

Pode-se assim observar que a função $T_{1}$ atende às hipóteses do lema 3.2.2, onde a função $K$ : $M(3) \longrightarrow \mathbb{R}$ mencionada é:

$$
K(A):=\frac{1}{2} \int_{\mathcal{B}}\|A u+v\|^{2} d m,
$$

facilitando o cálculo das derivadas desse termo.

Para calcular a derivada de $K$, considerando a equivalência de normas sobre espaços vetoriais de dimensão finita, $\forall H \in M(3)-\{0\}$ pequeno, temos

$$
\begin{gathered}
\frac{K(\Omega+H)-K(\Omega)-\int_{\mathcal{B}}\langle\Omega u+v, H u\rangle d m}{\|H\|}=\int_{\mathcal{B}} \frac{\|H u\|^{2}}{\|H\|} d m \leq \frac{\|H\|_{\text {sup }}^{2}}{\|H\|} \int_{\mathcal{B}}\|u\|^{2} d m \\
\leq \alpha\|H\| \int_{\mathcal{B}}\|u\|^{2} d m \stackrel{H \rightarrow 0}{\longrightarrow} 0 .
\end{gathered}
$$

Ou seja,

$$
d_{\Omega} K(H)=\int_{\mathcal{B}}\langle\Omega u+v, H u\rangle d m .
$$

Logo, denotando por $E_{i j}$ a matriz com entrada $i j$ igual a 1 e todas as outras nulas, $e_{i}$ o $i$-ésimo vetor da base canônica de $\mathbb{R}^{3}, \forall w \in \mathbb{R}^{3}$ temos,

$$
\begin{gathered}
\left.\frac{\partial K}{\partial \Omega} w=\sum_{i, j=1}^{3}\left(d_{\Omega} K\left(E_{i j}\right)\right) E_{i j} w=\sum_{i, j=1}^{3}\left(\int_{\mathcal{B}}\left\langle\Omega u+v, u_{j} e_{i}\right\rangle d m\right)\right) w_{j} e_{i}= \\
=\int_{\mathcal{B}}\left(\sum_{i, j=1}^{3}(\Omega u+v)_{i} u_{j} w_{j} e_{i}\right) d m=\int_{\mathcal{B}} \sum_{i=1}^{3}\left(\sum_{j=1}^{3}\left((\Omega u+v) u^{T}\right)_{i j} w_{j}\right) e_{i} d m= \\
=\sum_{i=1}^{3} \int_{\mathcal{B}}\left((\Omega u+v) u^{T} w\right)_{i} d m e_{i}=\left(\int_{\mathcal{B}}(\Omega u+v) u^{T} d m\right) w \Rightarrow \frac{\partial K}{\partial \Omega}=\int_{\mathcal{B}}(\Omega u+v) u^{T} d m .
\end{gathered}
$$

Onde a notação $a b^{T} \in \operatorname{Lin}\left(\mathbb{R}^{3}\right), \quad a, b \in \mathbb{R}^{3}$ foi usada para denotar o operador: $a b^{T} h:=(b \cdot h) a$ (adaptada à notação de matrizes).

Portanto,

$$
A(t)=\int_{\mathcal{B}}\left((\Omega u+v) u^{T}\right)_{a} d m
$$

O cálculo do termo correspondente à função $T_{2}$ é mais direto.

$$
T_{2}(Y, y, u, \dot{Y}, \dot{y}, v)=\int_{\mathcal{B}}\langle\dot{Y} u+Y v, \dot{y}\rangle d m
$$

Claramente

$$
\frac{T_{2}(\dot{Y}+H)-T_{2}(\dot{Y})-\int_{\mathcal{B}}\langle H u, \dot{y}\rangle d m}{\|H\|}=\frac{T_{2}(Y+H)-T_{2}(Y)-\int_{\mathcal{B}}\langle H v, \dot{y}\rangle d m}{\|H\|}=0 .
$$

Ou seja,

$$
d_{\dot{Y}} T_{2}(H)=\int_{\mathcal{B}}\langle H u, \dot{y}\rangle d m, \quad d_{Y} T_{2}(H)=\int_{\mathcal{B}}\langle H v, \dot{y}\rangle d m .
$$


Portanto, $\forall w \in \mathbb{R}^{3}$,

$$
\begin{aligned}
& \left.\frac{\partial T_{2}}{\partial \dot{Y}} w=\sum_{i, j=1}^{3}\left(d_{\dot{Y}} T_{2}\left(E_{i j}\right)\right) E_{i j} w=\sum_{i, j=1}^{3}\left(\int_{\mathcal{B}}\left\langle E_{i j} u, \dot{y}\right\rangle d m\right)\right) E_{i j} w=\int_{\mathcal{B}}\left(\sum_{i, j=1}^{3}\left\langle u_{j} e_{i}, \dot{y}\right\rangle w_{j} e_{i}\right) d m= \\
& \int_{\mathcal{B}}\left(\sum_{i, j=1}^{3} u_{j} \dot{y}_{i} w_{j} e_{i}\right) d m=\sum_{i, j=1}^{3}\left(\int_{\mathcal{B}}\left(\dot{y} u^{T}\right)_{i j} d m\right) w_{j} e_{i}=\sum_{i, j=1}^{3}\left(\int_{\mathcal{B}} \dot{y} u^{T} d m\right)_{i j} w_{j} e_{i}=\left(\int_{\mathcal{B}} \dot{y} u^{T} d m\right) w .
\end{aligned}
$$

Logo,

$$
\frac{d}{d t} \frac{\partial T_{2}}{\partial \dot{Y}}=\int_{\mathcal{B}} \ddot{y} u^{T}+\dot{y} v^{T} d m
$$

E, reproduzindo o mesmo cálculo para $\partial T_{2} / \partial Y$, conclui-se que:

$$
\begin{gathered}
\frac{\partial T_{2}}{\partial Y}=\int_{\mathcal{B}} \dot{y} v^{T} d m \Rightarrow \frac{d}{d t} \frac{\partial T_{2}}{\partial \dot{Y}}-\frac{\partial T_{2}}{\partial Y}=\int_{\mathcal{B}} \ddot{y} u^{T} d m \\
\Rightarrow P_{Y}\left(\frac{d}{d t} \frac{\partial T_{2}}{\partial \dot{Y}}-\frac{\partial T_{2}}{\partial Y}\right)=Y \int_{\mathcal{B}}\left(Y^{-1} \ddot{y} u^{T}\right)_{a} d m
\end{gathered}
$$

Assim, resulta da equação (3.12), fazendo uso de (3.16) e (3.18):

$$
\begin{gathered}
P_{Y}\left(\frac{d}{d t}\left(\frac{\partial T}{\partial \dot{Y}}\right)-\frac{\partial T}{\partial Y}\right)=P_{Y}\left(\frac{d}{d t}\left(\frac{\partial T_{1}}{\partial \dot{Y}}\right)-\frac{\partial T_{1}}{\partial Y}\right)+P_{Y}\left(\frac{d}{d t}\left(\frac{\partial T_{2}}{\partial \dot{Y}}\right)-\frac{\partial T_{2}}{\partial Y}\right)=-P_{Y}\left(\frac{\partial V}{\partial Y}\right) . \\
\Rightarrow Y\left(\frac{d A}{d t}(t)+[\Omega, A(t)]\right)+Y \int_{\mathcal{B}}\left(Y^{-1} \ddot{y} u^{T}\right)_{a}=-Y\left(Y^{-1} \frac{\partial V}{\partial Y}\right)_{a} \\
\Rightarrow \frac{d A}{d t}(t)+[\Omega, A(t)]+\int_{\mathcal{B}}\left(Y^{-1} \ddot{y} u^{T}\right)_{a}=-\left(Y^{-1} \frac{\partial V}{\partial Y}\right)_{a}
\end{gathered}
$$

Passemos agora ao desenvolvimento da equação (3.13). Esta é equivalente a:

$$
\frac{d}{d t}\left(\frac{\delta T}{\delta v}\right)-\frac{\delta T}{\delta u}=-\frac{\delta V}{\delta u}
$$

Para todos $v \in \mathcal{C}, \eta \in \mathcal{V}$, temos:

$$
\delta_{v} T(\eta)=\left.\frac{1}{2} \frac{d}{d \epsilon} \int_{\mathcal{B}}\left\|\Omega u+\phi_{\epsilon}+Y^{T} \dot{y}\right\|^{2} d m\right|_{\epsilon=0}=\int_{\mathcal{B}}\left\langle\Omega u+v+Y^{T} \dot{y}, \eta\right\rangle d m .
$$

Portanto, pela definição, existe a função $\delta T / \delta v \in \mathcal{C}$, cuja expressão é,

$$
\frac{\delta T}{\delta v}=\Omega u+v+Y^{T} \dot{y} \Rightarrow \frac{d}{d t} \frac{\delta T}{\delta v}=\dot{Y}^{T} \dot{Y} u+Y^{T} \ddot{Y} u+Y^{T} \dot{Y} u_{t}+u_{t t}+\dot{Y}^{T} \dot{y}+Y^{T} \ddot{y}
$$

Analogamente, $\forall u \in \mathcal{C}, \eta \in \mathcal{V}$ :

$$
\delta_{u} T(\eta)=\left.\frac{1}{2} \frac{d}{d \epsilon} \int_{\mathcal{B}}\left\|\Omega \phi_{\epsilon}+v+Y^{T} \dot{y}\right\|^{2} d m\right|_{\epsilon=0}=\int_{\mathcal{B}}\left\langle\Omega u+v+Y^{T} \dot{y}, \Omega \eta\right\rangle d m .
$$

Assim, lembrando que a matriz $\Omega$ é antissimétrica, decorre que:

$$
\frac{\delta T}{\delta u}=\Omega^{T}\left(\Omega u+v+Y^{T} \dot{y}\right)=-\Omega^{2} u-\Omega v+\dot{Y}^{T} \dot{y} .
$$


Portanto, resulta da equação (3.13):

$$
u_{t t}+2 \Omega u_{t}+\dot{\Omega} u+\Omega^{2} u+Y^{T} \ddot{y}=-\frac{\delta V}{\delta u}
$$

Para calcular a equação (3.14), considere $\forall w \in \mathbb{R}^{3}-\{0\}$ :

$$
\frac{T(\dot{y}+w)-T(\dot{y})-\int_{\mathcal{B}}\langle Y(\Omega u+v)+\dot{y}, w\rangle d m}{\|w\|}=\frac{\int_{\mathcal{B}}\|w\|^{2} d m}{\|w\|}=m(\mathcal{B})\|w\| \stackrel{w \rightarrow 0}{\longrightarrow} 0 .
$$

Portanto, $d_{\dot{y}} T(w)=\left\langle\int_{\mathcal{B}} Y(\Omega u+v)+\dot{y} d m, w\right\rangle$. Ou seja,

$$
\begin{gathered}
\frac{\partial T}{\partial \dot{y}}=\int_{\mathcal{B}} Y(\Omega u+v)+\dot{y} d m \Rightarrow \frac{d}{d t} \frac{\partial T}{\partial \dot{y}}=\int_{\mathcal{B}} \dot{Y}(\Omega u+v)+Y\left(\dot{\Omega} u+\Omega u_{t}+u_{t t}\right)+\ddot{y} d m= \\
=\int_{\mathcal{B}} Y\left(\Omega^{2} u+\Omega v\right)+Y\left(\dot{\Omega} u+\Omega u_{t}+u_{t t}\right)+\ddot{y} d m .
\end{gathered}
$$

Pois $\dot{Y}=Y \Omega$.

Desde que $\partial T / \partial y=0$ a equação (3.14) se reduz a:

$$
\int_{\mathcal{B}} u_{t t}+\Omega^{2} u+2 \Omega u_{t}+\dot{\Omega} u+Y^{T} \ddot{y} d m=-Y^{T} \frac{\partial V}{\partial y}
$$

Consideraremos sistemas mecânicos que apresentam forças dissipativas, modeladas matematicamente como gradientes de uma função das velocidades associadas às coordenadas que os descrevem. Como mencionado anteriormente, as equações de movimento são obtidas através de uma alteração no sistema (3.12), (3.13) e (3.14).

Neste contexto, faz-se uso de uma função dissipação (de Rayleigh) $\mathcal{D}: T M \longrightarrow \mathbb{R}$, suave, tal que a evolução temporal do sistema é solução de:

$$
\begin{gathered}
P_{Y}\left(\frac{d}{d t}\left(\frac{\partial L}{\partial \dot{Y}}\right)-\frac{\partial L}{\partial Y}+\frac{\partial \mathcal{D}}{\partial \dot{Y}}\right)=0 \\
\frac{d}{d t}\left(\frac{\delta L}{\delta v}\right)-\frac{\delta L}{\delta u}+\frac{\delta \mathcal{D}}{\delta v}=0 \\
\frac{d}{d t}\left(\frac{\partial L}{\partial \dot{y}}\right)-\frac{\partial L}{\partial y}+\frac{\partial \mathcal{D}}{\partial \dot{y}}=0
\end{gathered}
$$

Todos estes resultados apresentados são agora resumidos no seguinte teorema:

Teorema 3.2.2. (Baillieul \& Levi, 1987)

A evolução temporal do sistema mecânico em consideração, com energia potencial $V$ e função dissipação $\mathcal{D}$ é solução do seguinte sistema de equações

$$
\begin{gathered}
\frac{d A}{d t}(t)+[\Omega, A(t)]+\int_{\mathcal{B}}\left(Y^{-1} \ddot{y} u^{T}\right)_{a}=-\left(Y^{-1} \frac{\partial V}{\partial Y}\right)_{a}-\left(Y^{-1} \frac{\partial \mathcal{D}}{\partial \dot{Y}}\right)_{a} \\
u_{t t}+2 \Omega u_{t}+\dot{\Omega} u+\Omega^{2} u+Y^{-1} \ddot{y}=-\frac{\delta V}{\delta u}-\frac{\delta \mathcal{D}}{\delta v} \\
\int_{\mathcal{B}} u_{t t}+\Omega^{2} u+2 \Omega u_{t}+\dot{\Omega} u+Y^{-1} \ddot{y} d m=-Y^{-1} \frac{\partial V}{\partial y}-Y^{-1} \frac{\partial \mathcal{D}}{\partial \dot{y}}
\end{gathered}
$$

Onde $A(t)$ é dado por (3.16). 
Observação 3.2.4. Na presença de forças devidas a interações externas, o movimento é descrito da mesma forma, com um termo adicional depois do sinal de igual em cada uma das equaçôes apresentadas.

A dificuldade consiste em, caso a força não venha de um potencial conhecido, separar a força total em sua contribuição ao longo de cada uma das coordenadas.

É conveniente escrever estas equações numa forma alternativa. Para isso, defina a seguinte função (isomorfismo linear): $S: \operatorname{Ant}(3) \longrightarrow \mathbb{R}^{3}$,

$$
S(B)=S\left(\left(\begin{array}{ccc}
0 & -b_{3} & b_{2} \\
b_{3} & 0 & -b_{1} \\
-b_{2} & b_{1} & 0
\end{array}\right)\right)=\left(\begin{array}{l}
b_{1} \\
b_{2} \\
b_{3}
\end{array}\right)
$$

O seguinte lema é facilmente verificado escrevendo-se explicitamente em componentes.

Lema 3.2.3. Dados $A, B \in A n t(3)$ e $w_{1}, w_{2} \in \mathbb{R}^{3}$, valem as seguintes identidades:

(i) $S([A, B])=S(A) \times S(B)$.

(ii) $S\left(w_{1} w_{2}^{T}-w_{2} w_{1}^{T}\right)=w_{2} \times w_{1}$, e portanto, $S\left(\left(w_{1} w_{2}^{T}\right)_{a}\right)=\frac{1}{2} w_{2} \times w_{1}$.

(iii) $A w_{1}=S(A) \times w_{1}$.

Com ajuda desse lema, as referidas equações são reescritas. A constatação das seguintes expressões é direta.

Corolário 3.2.1. As equações (3.21), (3.22) e (3.23) são equivalentes a:

$$
\begin{gathered}
\dot{a}(t)+\omega \times a(t)+\int_{\mathcal{B}} u \times Y^{-1} \ddot{y} d m=-2 S\left(\left(Y^{-1} \frac{\partial V}{\partial Y}\right)_{a}\right)-2 S\left(\left(Y^{-1} \frac{\partial \mathcal{D}}{\partial \dot{Y}}\right)_{a}\right) \\
u_{t t}+2 \omega \times u_{t}+\dot{\omega} \times u+\omega \times(\omega \times u)+Y^{-1} \ddot{y}=-\frac{\delta V}{\delta u}-\frac{\delta \mathcal{D}}{\delta v} \\
\int_{\mathcal{B}} u_{t t}+\omega \times(\omega \times u)+2 \omega \times u_{t}+\dot{\omega} \times u+Y^{-1} \ddot{y} d m=-Y^{-1} \frac{\partial V}{\partial y}-Y^{-1} \frac{\partial \mathcal{D}}{\partial \dot{y}}
\end{gathered}
$$

Onde $a(t):=\int_{\mathcal{B}} u \times\left(u_{t}+\omega \times u\right) d m=2 S(A)$ e $\omega(t):=S(\Omega)$ que será denominada velocidade angular no referencial do corpo.

Somente para simplificar a notação, defina a seguinte derivação: $D b(t):=\partial b / \partial t+\omega(t) \times b(t)$, $\forall b \in C^{1}$.

Corolário 3.2.2. Com esta notação, as equações (3.24), (3.25) e (3.26) sẫo escritas como:

$$
\begin{gathered}
\int_{\mathcal{B}} u \times\left(D^{2} u+Y^{-1} \ddot{y}\right) d m=-2 S\left(\left(Y^{-1} \frac{\partial V}{\partial Y}\right)_{a}\right)-2 S\left(\left(Y^{-1} \frac{\partial \mathcal{D}}{\partial \dot{Y}}\right)_{a}\right) \\
D^{2} u+Y^{-1} \ddot{y}=-\frac{\delta V}{\delta u}-\frac{\delta \mathcal{D}}{\delta v} \\
\int_{\mathcal{B}} Y D^{2} u+\ddot{y} d m=-\frac{\partial V}{\partial y}-\frac{\partial \mathcal{D}}{\partial \dot{y}}
\end{gathered}
$$

Demonstração. De fato, $D^{2} u=D\left(u_{t}+\omega \times u\right)=u_{t t}+\dot{\omega} \times u+2 \omega \times u_{t}+\omega \times(\omega \times u)$.

Além disso,

$\dot{a}+\omega \times a=\int_{\mathcal{B}} u_{t} \times(\omega \times u)+u \times\left(u_{t t}+\dot{\omega} \times u+\omega \times u_{t}\right)+\omega \times\left(u \times u_{t}\right)+\omega \times(u \times(\omega \times u)) d m$. 
Pela identidade de Jacobi para o produto vetorial,

$\omega \times\left(u \times u_{t}\right)+u_{t} \times(\omega \times u)+u \times\left(u_{t} \times \omega\right)=0 \Rightarrow \omega \times\left(u \times u_{t}\right)+u_{t} \times(\omega \times u)=u \times\left(\omega \times u_{t}\right)$

$\omega \times(u \times(\omega \times u))+(\omega \times u) \times(\omega \times u)+u \times((\omega \times u) \times \omega)=0 \Rightarrow \omega \times(u \times(\omega \times u))=u \times(\omega \times(\omega \times u))$

Logo,

$$
\dot{a}+\omega \times a=\int_{\mathcal{B}} u \times\left(u_{t t}+\dot{\omega} \times u+2 \omega \times u_{t}+\omega \times(\omega \times u)\right) d m=\int_{\mathcal{B}}\left(u \times D^{2} u\right) d m
$$

Definição 3.2.2. Suponha que a evolução temporal de um sistema seja dada pelas equações (3.24), (3.25) e (3.26). Considere as propriedades:

(i) A configuração $u(z, t)=u(z)$ é constante no tempo.

(ii) As funções $\partial V / \partial Y, \partial \mathcal{D} / \partial \dot{Y}$ são nulas ao longo desta solução.

(iii) As funções $\partial V / \partial y, \partial \mathcal{D} / \partial \dot{y}$, são nulas ao longo desta solução.

Se a solução satisfizer as propriedades (ii) e (iii), ela será denominada solução livre de torque.

Se a solução satisfizer as três propriedades, ela será denominada solução estacionária.

Suponha agora que o corpo execute um movimento que seja livre de torque. Observemos o movimento do (chamado) centro de massa do sistema sob estas hipóteses. Da equação (3.23), inferese:

$$
\frac{d^{2}}{d t^{2}} Y \int_{\mathcal{B}} u+Y^{T} y d m=0 \Rightarrow Y^{T} y=-C(t)+c_{1}+t c_{2}
$$

onde $c_{1}, c_{2}$ são constantes relacionadas às condições iniciais, $C(t):=\left(\int_{\mathcal{B}} u d m\right) / m$, e $m:=\int_{\mathcal{B}} d m$ a massa total.

Em particular, $(d / d t)(Y C+y)=c_{2}$, o que é denominado conservação do momento linear total $(m(d / d t)(Y C+y)$, que está no referencial inercial). O vetor $Y C+y$ é denominado o centro de massa do sistema, no referencial inercial.

Pode-se trivialmente fazer as constantes $c_{1}=c_{2}=0$ escolhendo adequadamente a origem do referencial inercial.

Suponha agora que a solução seja estacionária. Logo, $C(t)$ é constante.

Defina: $\zeta:(\mathcal{B}-C) \longrightarrow \mathbb{R}^{3}, \zeta(x): \overline{=u(x+C)}-C$. Logo $\zeta(\cdot)$ é difeo sobre sua imagem.

Defina também:

$$
\tilde{a}(t):=\int_{\mathcal{B}-C} \zeta(x) \times(\omega(t) \times \zeta(x)) d m .
$$

Desta forma,

$$
\begin{aligned}
a(t) & =\int_{\mathcal{B}}(\zeta(x-C)+C) \times(\omega \times(\zeta(x-C)+C)) d m=\int_{\mathcal{B}} \zeta(x-C) \times(\omega \times \zeta(x-C)) d m+ \\
& +\left(\int_{\mathcal{B}} \zeta(x-C) d m\right) \times(\omega \times C)+C \times\left(\omega \times\left(\int_{\mathcal{B}} \zeta(x-C) d m\right)+m C \times(\omega \times C) .\right.
\end{aligned}
$$

Mas,

$$
\int_{\mathcal{B}} \zeta(x-C) d m=\int_{\mathcal{B}}(u(x)-C) d m=m(C-C)=0 .
$$

Logo,

$$
\begin{gathered}
a(t)=\tilde{a}(t)+C \times(\omega \times C) \\
\Rightarrow \dot{a}+\omega \times a=\dot{\tilde{a}}+m C \times(\dot{\omega} \times C)+\omega \times \tilde{a}+m \omega \times(C \times(\omega \times C))=-m C \times Y^{T} \ddot{y} .
\end{gathered}
$$


Onde a última igualdade é justificada pela equação (3.24).

Desde que $y=-Y C, \ddot{y}=-Y D^{2} C=-Y(\dot{\omega} \times C+\omega \times(\omega \times C)), \Rightarrow-C \times Y^{T} \ddot{y}=C \times(\dot{\omega} \times$ $C)+C \times(\omega \times(\omega \times C)))$.

Portanto,

$$
\dot{\tilde{a}}+\omega \times \tilde{a}=0 .
$$

Observe que, neste regime de movimento,

$$
\tilde{a}(t)=-\int_{\mathcal{B}-C} \zeta(x) \times(\zeta(x) \times \omega(t))=I_{\infty} \omega(t) .
$$

Onde, $I_{\infty}:=-\left(\int_{\mathcal{B}-C}\left(S^{-1}(\zeta(x))\right)^{2} d m\right)$ é um operador linear (constante no tempo).

Logo, para soluções estacionárias, $\omega(t)$ deve satisfazer:

$$
I_{\infty} \dot{\omega}=\left(I_{\infty} \omega\right) \times \omega .
$$

O vetor $a(t)$ que surge na equação (3.24) está relacionado ao momento angular total no referencial do corpo. Note que ele é simétrico.

Para uso futuro, é conveniente enunciar os resultados desta discussão na forma de um lema.

Lema 3.2.4. Para soluçôes estacionárias, as equações (3.24), (3.25), (3.26) são equivalentes a:

$$
\begin{gathered}
I_{\infty} \dot{\omega}(t)=I_{\infty} \omega(t) \times \omega \\
\dot{\omega} \times u+\omega \times(\omega \times u)=-\frac{\delta V}{\delta u}-\frac{\delta \mathcal{D}}{\delta v} \\
y(t)=-Y(t) C
\end{gathered}
$$

Se o referencial inercial é adequadamente escolhido.

Novamente, pensando no corpo como uma extensão de um sistema de partículas, é natural definir-se o momento angular no referencial inercial como:

$$
M(t):=\int_{\mathcal{B}} U \times U_{t} d m=\int_{\mathcal{B}}(Y u+y) \times \frac{\partial}{\partial t}(Y u+y) d m=\int_{\mathcal{B}}(Y u+y) \times\left(Y\left(u_{t}+\omega \times u\right)+\dot{y}\right) d m .
$$

Logo, no referencial do corpo,

$$
Y^{T} M=a(t)+Y^{T} y \times \int_{\mathcal{B}}\left(D u+Y^{T} \dot{y}\right) d m .
$$

Vemos assim que $a(t)$ está relacionado com o momento angular no referencial do corpo.

Calculando:

$\frac{d}{d t} M(t)=\int_{\mathcal{B}}(Y u+y) \times\left(Y D^{2} u+\ddot{y}\right) d m=Y \int_{\mathcal{B}} u \times\left(D^{2} u+Y^{T} \ddot{y}\right) d m+y \times \int_{\mathcal{B}}\left(Y D^{2} u+\ddot{y}\right) d m=0$.

Vemos assim que no caso de solução livre de torque, das equações (3.27) e (3.29) decorre que o momento angular total é conservado. Fato que muitas vezes é colocado como postulado em teoria da elasticidade.

Tratemos agora a questão da energia para corpos deformáveis.

Como usual em mecânica Lagrangiana, há uma expressão para a energia total do sistema. É análoga à transformação de Legendre para o caso de dimensão finita:

$$
E=\left\langle\dot{Q}, \frac{\partial L}{\partial \dot{Q}}\right\rangle-L(Q, \dot{Q}) .
$$


Logo,

$$
E:=\left\langle\dot{y}, \frac{\partial L}{\partial \dot{y}}\right\rangle+\left\langle u_{t}, \frac{\partial L}{\partial v}\right\rangle+\left\langle\dot{Y}, \frac{\partial L}{\partial \dot{Y}}\right\rangle-T+V .
$$

E, pelas equações (3.10), (3.15) e (3.17), temos:

$$
\begin{gathered}
\left\langle\dot{y}, \frac{\partial L}{\partial \dot{y}}\right\rangle=\left(Y^{T} \dot{y}\right) \cdot \int_{\mathcal{B}} D u+Y^{T} \dot{y} d m=\int_{\mathcal{B}}\left(Y^{T} \dot{y}\right) \cdot\left(D u+Y^{T} \dot{y}\right) d m \\
\left\langle v, \frac{\delta L}{\delta v}\right\rangle=\int_{\mathcal{B}} v \cdot\left(D u+Y^{T} \dot{y}\right) d m \\
\left\langle\dot{Y}, \frac{\partial L}{\partial \dot{Y}}\right\rangle=\int_{\mathcal{B}} Y^{T} \dot{Y} u \cdot\left(D u+Y^{T} \dot{y}\right) d m=\int_{\mathcal{B}} \Omega u \cdot\left(D u+Y^{T} \dot{y}\right) d m .
\end{gathered}
$$

Logo, como usual,

$$
E=\frac{1}{2} \int_{\mathcal{B}}\left\|D u+Y^{T} \dot{y}\right\|^{2} d m+V=T+V .
$$

A derivada temporal de $E$ ao longo das soluções das equações no teorema (3.2.2) fica (usando a equação (3.37)):

$$
\begin{gathered}
\dot{E}=\left\langle\ddot{y}, \frac{\partial T}{\partial \dot{y}}\right\rangle+\left\langle u_{t t}, \frac{\delta T}{\delta v}\right\rangle+\left\langle\ddot{Y}, \frac{\partial T}{\partial \dot{Y}}\right\rangle+\left\langle\dot{y}, \frac{d}{d t}\left(\frac{\partial T}{\partial \dot{y}}\right)\right\rangle+\left\langle u_{t}, \frac{d}{d t}\left(\frac{\delta T}{\delta v}\right)\right\rangle+\left\langle\dot{Y}, \frac{d}{d t}\left(\frac{\partial T}{\partial \dot{Y}}\right)\right\rangle- \\
-\left\langle\ddot{y}, \frac{\partial T}{\partial \dot{y}}\right\rangle-\left\langle u_{t t}, \frac{\delta T}{\delta v}\right\rangle-\left\langle\ddot{Y}, \frac{\partial T}{\partial \dot{Y}}\right\rangle-\left\langle\dot{y}, \frac{\partial T}{\partial y}\right\rangle-\left\langle u_{t}, \frac{\delta T}{\delta u}\right\rangle-\left\langle\dot{Y}, \frac{\partial T}{\partial Y}\right\rangle+ \\
+\left\langle\dot{y}, \frac{\partial V}{\partial y}\right\rangle+\left\langle u_{t}, \frac{\delta V}{\delta u}\right\rangle+\left\langle\dot{Y}, \frac{\partial V}{\partial Y}\right\rangle=\left\langle\dot{y}, \frac{d}{d t}\left(\frac{\partial T}{\partial \dot{y}}\right)-\frac{\partial T}{\partial y}+\frac{\partial V}{\partial y}\right\rangle+\left\langle u_{t}, \frac{d}{d t}\left(\frac{\delta T}{\delta v}\right)-\frac{\delta T}{\delta u}+\frac{\delta V}{\delta u}\right\rangle+ \\
+\left\langle\dot{Y}, \frac{d}{d t}\left(\frac{\partial T}{\partial \dot{Y}}\right)-\frac{\partial T}{\partial Y}+\frac{\partial V}{\partial Y}\right\rangle .
\end{gathered}
$$

Logo,

$$
\dot{E}=-\left\langle\dot{y}, \frac{\partial \mathcal{D}}{\partial \dot{y}}\right\rangle-\left\langle u_{t}, \frac{\delta \mathcal{D}}{\delta v}\right\rangle-\left\langle\dot{Y}, \frac{\partial \mathcal{D}}{\partial \dot{Y}}\right\rangle
$$

Este cálculo justifica de certa forma a correspondência que se faz da função $\mathcal{D}$ com a variação da energia. No entanto, sob as hipóteses feitas, não é possível garantir que a expressão acima seja não positiva, o que estaria de acordo com o contexto de dissipação, onde a energia mecânica perdida, ou convertida em outra forma de energia, não é convertida à forma inicial.

Observação 3.2.5. Um caso particular interessante desse sistema de equações é o corpo rígido ideal onde, por definição, não há o grau de liberdade relacionado à deformação, $u(t, x) \equiv x, \forall t, x$ $e V, \mathcal{D} \equiv 0$ (já que não há deformação). Neste caso podemos tomar a coordenada y sobre o centro de massa do corpo, ficando constante no tempo, ou seja, eliminando este grau de liberdade. Dessa forma, (3.28) e (3.29) não fazem parte das equações de movimento, e o operador $I_{\infty}$ é exatamente o conhecido por tensor de inércia do corpo rígido.

Neste caso, também temos:

$$
a(t)=-\int_{B} x \times(x \times \omega) d m=I \omega .
$$

Que é o momento angular usado na seção (2.2).

Deve-se ressaltar que esta observação consiste apenas na conclusão: fazendo-se estas exigências sobre as variáveis, consegue-se resgatar o problema usual do corpo rígido ideal sem forças externas, podendo este ser visto neste contexto. Isso indica que essas equações podem realmente ser adequadas para a situação proposta. 
A seguir, será necessário o seguinte lema.

Lema 3.2.5. Todos autovalores de $I_{\infty}, I_{1} \geq I_{2} \geq I_{3}$, são positivos. Além disso, defina:

$$
\alpha_{1}:=\frac{I_{2}-I_{3}}{I_{1}} \quad \alpha_{2}:=\frac{I_{3}-I_{1}}{I_{2}} \quad \alpha_{3}:=\frac{I_{1}-I_{2}}{I_{3}} .
$$

Dessa forma, valem as identidades e as desigualdades:

(i) $\alpha_{1}+\alpha_{2}+\alpha_{3}+\alpha_{1} \alpha_{2} \alpha_{3}=0$;

(ii) $I_{1}^{2} \alpha_{1}+I_{2}^{2} \alpha_{2}+I_{3}^{2} \alpha_{3}=0$.

(iii) $I_{1} \alpha_{1}+I_{2} \alpha_{2}+I_{3} \alpha_{3}=0$.

(iv) $\left|\alpha_{i}\right|<1, \forall i=1,2,3$.

Demonstração. Como $I_{\infty}$ é simétrica, existe uma base de $\mathbb{R}^{3}$ formada por seus autovetores. $I_{i}$, $i=1,2,3$ são seus correspondentes autovalores.

Tome $v \in \mathbb{R}^{3}$ vetor não nulo qualquer. Assim,

$$
\left\langle v, I_{\infty} v\right\rangle=-\int_{\mathcal{B}-C}\langle v, \zeta \times(\zeta \times v)\rangle d m=\int_{\mathcal{B}-C}\|\zeta \times v\|^{2} d m \geq 0 .
$$

Além disso, a expressão acima se anula se e somente $v \times \zeta(z)=0, \forall z \in \mathcal{B}-C$, o que é equivalente a $\zeta(z)=\lambda(z) v, \forall z \in \mathcal{B}-C$, e que contradiz o fato de $\zeta$ ser difeomorfismo. Logo $\left\langle v, I_{\infty} v\right\rangle>0, \forall v \in \mathbb{R}^{3}$, o que prova a primeira afirmação.

As identidades citadas resultam de cálculos diretos.

Seja $\left\{e_{i} \in \mathbb{R}^{3}, i=1,2,3\right\}$ uma base ortonormal de autovetores correspondentes a $I_{i}$, e $\zeta_{i} \in \mathbb{R}$ tais que $\zeta=\zeta_{1} e_{1}+\zeta_{2} e_{2}+\zeta_{3} e_{3}$.

Assim, se a permutação $\{1,2,3\} \rightarrow\{i, j, k\}$ é par,

$$
I_{i}=<e_{i}, I_{\infty} e_{i}>=\int_{\mathcal{B}-C}\left\|e_{i} \times \zeta\right\|^{2} d m=\int_{\mathcal{B}-C} \zeta_{j}^{2}+\zeta_{k}^{2} d m
$$

Logo,

$$
\alpha_{i}=\frac{I_{j}-I_{k}}{I_{i}}=\frac{\int_{\mathcal{B}-C} \zeta_{j}^{2}-\zeta_{k}^{2} d m}{\int_{\mathcal{B}-C} \zeta_{j}^{2}+\zeta_{k}^{2} d m} \Longrightarrow\left|\alpha_{i}\right|<1
$$

O teorema a seguir determina as propriedades da velocidade angular para as soluções estacionárias.

Teorema 3.2.3. Para as soluções estacionárias, valem:

(i) A velocidade angular é constante, $\omega_{\infty}$.

(ii) O vetor $a(t)$ é constante, $a_{\infty}=I_{\infty} \omega_{\infty}$.

(iii) $\omega_{\infty}$ é autovetor de $I_{\infty}$.

Demonstração. A dificuldade do teorema reside na demonstração do item $(i)$.

Para tanto, observe que, por hipótese, $u$ é um difeomorfismo sobre sua imagem. Portanto, a função $\partial u / \partial z$ não se anula em $\mathcal{B}$.

Assim, tomando a derivada parcial da equação (3.33) com respeito a $z$ em um ponto $p \in \mathcal{B}$, e fazendo $h:=\partial u(p) / \partial z$, temos:

$$
\omega \times(\omega \times h)+\dot{\omega} \times h=\bar{c} .
$$


Onde $\bar{c}:=-\partial_{z}(\delta V / \delta u)$.

Seja $H:=S^{-1}(h)$. Dessa forma, a equação acima é equivalente a:

$$
\omega \times(H \omega)+H \dot{\omega}+\bar{c}=0 .
$$

Como $h$ é não nulo, tome $v \in \mathbb{R}^{3}$, qualquer unitário ortogonal a $h$, e seja $w:=(h \times v) /\|h\|$. Ou seja, $\{v, w, h /\|h\|\}$ é base ortonormal positiva.

É um cálculo direto verificar que $H(v)=-\|h\| w, H(w)=\|h\| v, H(h /\|h\|)=0$. Ou seja se $G$ é a matriz de mudança de base (ortogonal) dada por:

$$
G=\left(\begin{array}{ccc}
v_{1} & v_{2} & v_{3} \\
w_{1} & w_{2} & w_{3} \\
h_{1} /\|h\| & h_{2} /\|h\| & h_{3} /\|h\|
\end{array}\right) \Rightarrow G H G^{T}=\left(\begin{array}{ccc}
0 & \|h\| & 0 \\
-\|h\| & 0 & 0 \\
0 & 0 & 0
\end{array}\right) .
$$

Definindo, $\bar{\omega}:=G \omega$ e multiplicando a equação (3.39) à esquerda por $G$, temos:

$$
\bar{\omega} \times\left(G H G^{T} \bar{\omega}\right)+G H G^{T} \dot{\bar{\omega}}+G \bar{c}=0 \Leftrightarrow\left(\begin{array}{c}
\dot{\bar{\omega}}_{1}-\bar{\omega}_{2} \bar{\omega}_{3} \\
\dot{\bar{\omega}}_{2}+\bar{\omega}_{1} \bar{\omega}_{3} \\
\bar{\omega}_{1}^{2}+\bar{\omega}_{2}^{2}
\end{array}\right)=\left(\begin{array}{c}
c_{2} \\
-c_{1} \\
c_{3}
\end{array}\right) .
$$

Onde $\left(c_{1}, c_{2}, c_{3}\right)=G \bar{c} /\|h\|$.

Podem ocorrer então duas situações:

(i) $c_{1} \neq 0$ ou $c_{2} \neq 0$.

Sem perda de generalidade, assuma que $c_{1} \neq 0$.

Neste caso, multiplique as duas primeiras componentes do sistema (3.40) por $\bar{\omega}_{1}$ e $\bar{\omega}_{2}$, respectivamente e some-as:

$$
c_{2} \bar{\omega}_{1}-c_{1} \bar{\omega}_{2}=\bar{\omega}_{1} \dot{\bar{\omega}}_{1}+\bar{\omega}_{2} \dot{\bar{\omega}}_{2}=\frac{1}{2} \frac{d}{d t}\left(\bar{\omega}_{1}^{2}+\bar{\omega}_{2}^{2}\right)=0 \Rightarrow \bar{\omega}_{2}=\frac{c_{2}}{c_{1}} \bar{\omega}_{1} \Rightarrow c_{3}=\left(1+\frac{c_{2}^{2}}{c_{1}^{2}}\right) \bar{\omega}_{1}^{2} .
$$

Ou seja, pela continuidade de $\omega, \bar{\omega}_{1}$ é constante. Logo, novamente pela terceira equação de (3.40), $\bar{\omega}_{2}$ é constante. E, pela segunda equação (desde que $\dot{\bar{\omega}}_{1}=0$ e foi suposto $c_{1} \neq 0$ ) obtemos que $\bar{\omega}_{3}$ é constante. Ou seja, $\bar{\omega}$ é constante, implicando (desde que $G$ é invertível) em $\omega$ ser constante.

(ii) $c_{1}=0$ e $c_{2}=0$.

Neste caso, sendo $x_{i} \in \mathbb{R}$ as coordenadas de $\omega(t)=\sum_{i} x_{i}(t) e_{i}$, e observando que $\omega$ também satisfaz à equação (3.32), temos:

$$
I_{\infty} \omega=\sum_{i=1}^{3} I_{i} x_{i} e_{i} \Rightarrow\left(I_{\infty} \omega\right) \times \omega=\left(I_{2}-I_{3}\right) x_{2} x_{3} e_{1}+\left(I_{3}-I_{1}\right) x_{1} x_{3} e_{2}+\left(I_{1}-I_{2}\right) x_{1} x_{2} e_{3} .
$$

Assim, a equação (3.32) nestas coordenadas fica:

$$
\left(\begin{array}{c}
\dot{x}_{1} \\
\dot{x}_{2} \\
\dot{x}_{3}
\end{array}\right)=\left(\begin{array}{c}
\alpha_{1} x_{2} x_{3} \\
\alpha_{2} x_{1} x_{3} \\
\alpha_{3} x_{1} x_{2}
\end{array}\right)
$$

Os coeficientes $\alpha_{i}$ foram definidos no lema (3.2.5).

Defina,

$$
\begin{gathered}
\tilde{M}:=I_{\infty} \omega, \quad \tilde{T}:=\frac{1}{2}\left\langle\omega, I_{\infty} \omega\right\rangle . \\
\Rightarrow \frac{d}{d t}\left(\tilde{M}^{2}\right)=\frac{d}{d t}\left(I_{1}^{2} x_{1}^{2}+I_{2}^{2} x_{2}^{2}+I_{3}^{2} x_{3}^{2}\right)=2\left(I_{1}^{2} \alpha_{1}+I_{2}^{2} \alpha_{2}+I_{3}^{2} \alpha_{3}\right) x_{1} x_{2} x_{3}=0
\end{gathered}
$$




$$
\Rightarrow \frac{d}{d t}(2 \tilde{T})=\frac{d}{d t}\left(I_{1} x_{1}^{2}+I_{2} x_{2}^{2}+I_{3} x_{3}^{2}\right)=2\left(I_{1} \alpha_{1}+I_{2} \alpha_{2}+I_{3} \alpha_{3}\right) x_{1} x_{2} x_{3}=0
$$

Pelas identidades $(i i)$ e (iii) do lema (3.2.5).

Assim, desde que $x_{i}$ e $\bar{\omega}_{i}(i=1,2,3)$ são coordenadas de $\omega$ em duas bases ortonormais, conclui-se de (3.40) que elas satisfazem:

$$
\begin{gathered}
I_{1}^{2} x_{1}^{2}+I_{2}^{2} x_{2}^{2}+I_{3}^{2} x_{3}^{2}=\tilde{M}^{2}=\text { constante } \\
I_{1} x_{1}^{2}+I_{2} x_{2}^{2}+I_{3} x_{3}^{2}=2 \tilde{T}=\text { constante } \\
\|\omega\|^{2}=x_{1}^{2}+x_{2}^{2}+x_{3}^{2}=\bar{\omega}_{1}^{2}+\bar{\omega}_{2}^{2}+\bar{\omega}_{3}^{2}=c_{3}^{2}+\bar{\omega}_{3}^{2}
\end{gathered}
$$

Por estas equaçôes, observamos que $\omega$ fica restrito à interseção de dois elipsóides e um cilindro. Assim, para concluir o teorema, resta somente provar a seguinte afirmação.

Afirmação: As equações (3.42) somente podem ser satisfeitas simultaneamente se $\omega$ é constante.

Prova:

Suponha que $\alpha_{1} \alpha_{2} \alpha_{3} \neq 0$. Assim, pela convenção do lema (3.2.5), $I_{1}>I_{2}>I_{3}>0$.

Portanto, das equações (3.42) decorre:

$$
I_{3}\left(I_{1}\left(c_{3}^{2}+\bar{\omega}_{3}^{2}\right)-2 T_{\text {cin }}\right)+\left(M^{2}-2 I_{1} T_{\text {cin }}\right)=\left(I_{3}-I_{2}\right)\left(I_{1}-I_{2}\right) x_{2}^{2} \Rightarrow \frac{d}{d t} \bar{\omega}_{3}^{2}=-2 \alpha_{1} \alpha_{2} \alpha_{3} x_{1} x_{2} x_{3}
$$

Mas de (3.41) e da terceira equação de (3.42),

$$
\frac{d}{d t} \bar{\omega}_{3}^{2}=2 \alpha_{1} \alpha_{2} \alpha_{3} x_{1} x_{2} x_{3} .
$$

Conclui-se disto que $x_{1} x_{2} x_{3} \equiv 0$.

Logo, para $i=1,2,3$ :

$$
\frac{d}{d t} x_{i}^{2}=2 \alpha_{i} x_{1} x_{2} x_{3} \equiv 0 \quad \Rightarrow\left\|x_{i}\right\|=\text { constante } \Rightarrow x_{i}=\text { constante } \quad \Rightarrow \omega=\text { constante } .
$$

Vejamos agora o caso em que $\alpha_{1} \alpha_{2} \alpha_{3}=0$.

Temos:

$$
\frac{d}{d t} \bar{\omega}_{3}^{2}=2 \alpha_{1} \alpha_{2} \alpha_{3} x_{1} x_{2} x_{3}=0 \quad \Rightarrow \bar{\omega}_{3}=\text { constante. }
$$

Se $\bar{\omega}_{3}=0$, a conclusão de que $\omega$ é constante é trivial. Analisemos então o caso contrário.

Sem perda de generalidade, suponha $\alpha_{1}=0$. Logo,

$$
\begin{gathered}
\dot{x}_{1}=\alpha_{1} x_{2} x_{3}=0 \quad \Rightarrow x_{1}=\text { constante } \\
I_{2}=I_{3} \quad \Rightarrow \alpha_{2}=-\alpha_{3} .
\end{gathered}
$$

Ou seja, obtemos os dois sistemas de equações:

$$
\begin{gathered}
\left(\begin{array}{c}
\dot{\bar{\omega}}_{1} \\
\dot{\bar{\omega}}_{2} \\
\dot{\bar{\omega}}_{3}
\end{array}\right)=\left(\begin{array}{c}
\bar{\omega}_{2} \bar{\omega}_{3} \\
-\bar{\omega}_{1} \bar{\omega}_{3} \\
0
\end{array}\right) \Longrightarrow\left(\begin{array}{c}
\ddot{\bar{\omega}}_{1} \\
\ddot{\bar{\omega}}_{2} \\
\ddot{\bar{\omega}}_{3}
\end{array}\right)=\left(\begin{array}{c}
-\bar{\omega}_{3}^{2} \bar{\omega}_{1} \\
-\bar{\omega}_{3}^{2} \bar{\omega}_{2} \\
0
\end{array}\right)=-\bar{\omega}_{3}^{2}\left(\begin{array}{c}
\bar{\omega}_{1} \\
\bar{\omega}_{2} \\
0
\end{array}\right) \\
\left(\begin{array}{c}
\dot{x}_{1} \\
\dot{x}_{2} \\
\dot{x}_{3}
\end{array}\right)=\left(\begin{array}{c}
\alpha_{2} x_{1} x_{3} \\
-\alpha_{2} x_{1} x_{2}
\end{array}\right) \Longrightarrow\left(\begin{array}{c}
\ddot{x}_{1} \\
\ddot{x}_{2} \\
\ddot{x}_{3}
\end{array}\right)=\left(\begin{array}{c}
0 \\
-\left(\alpha_{2} x_{1}\right)^{2} x_{2} \\
-\left(\alpha_{2} x_{1}\right)^{2} x_{3}
\end{array}\right)=-\left(\alpha_{2} x_{1}\right)^{2}\left(\begin{array}{c}
0 \\
x_{2} \\
x_{3}
\end{array}\right)
\end{gathered}
$$

Além disso, sabemos que existe uma matriz ortogonal $\tilde{G}$ tal que:

$$
\left(\begin{array}{c}
\bar{\omega}_{1} \\
\bar{\omega}_{2} \\
\bar{\omega}_{3}
\end{array}\right)=\tilde{G}\left(\begin{array}{c}
x_{1} \\
x_{2} \\
x_{3}
\end{array}\right) \Rightarrow\left(\begin{array}{c}
\bar{\omega}_{1} \\
\bar{\omega}_{2} \\
0
\end{array}\right)=-\frac{1}{\bar{\omega}_{3}^{2}}\left(\begin{array}{c}
\ddot{\bar{\omega}}_{1} \\
\ddot{\bar{\omega}}_{2} \\
\ddot{\bar{\omega}}_{3}
\end{array}\right)=-\frac{1}{\bar{\omega}_{3}^{2}} \tilde{G}\left(\begin{array}{c}
\ddot{x}_{1} \\
\ddot{x}_{2} \\
\ddot{x}_{3}
\end{array}\right)=\frac{\left(\alpha_{2} x_{1}\right)^{2}}{\bar{\omega}_{3}^{2}} \tilde{G}\left(\begin{array}{c}
0 \\
x_{2} \\
x_{3}
\end{array}\right)
$$




$$
\begin{gathered}
\Rightarrow\left(\begin{array}{c}
0 \\
0 \\
-\bar{\omega}_{3}
\end{array}\right)+\left(\begin{array}{c}
\bar{\omega}_{1} \\
\bar{\omega}_{2} \\
\bar{\omega}_{3}
\end{array}\right)=\frac{\left(\alpha_{2} x_{1}\right)^{2}}{\bar{\omega}_{3}^{2}} \tilde{G}\left\{\left(\begin{array}{c}
x_{1} \\
x_{2} \\
x_{3}
\end{array}\right)+\left(\begin{array}{c}
-x_{1} \\
0 \\
0
\end{array}\right)\right\} \\
\Rightarrow\left(\begin{array}{c}
\left(\bar{\omega}_{3}^{2}-\left(\alpha_{2} x_{1}\right)^{2}\right) \bar{\omega}_{1} \\
\left(\bar{\omega}_{3}^{2}-\left(\alpha_{2} x_{1}\right)^{2}\right) \bar{\omega}_{2} \\
-\left(\alpha_{2} x_{1}\right)^{2} \bar{\omega}_{3}
\end{array}\right)=\tilde{G}\left(\begin{array}{c}
-\alpha_{2}^{2} x_{1}^{3} \\
0 \\
0
\end{array}\right) \Rightarrow\left(\begin{array}{c}
\left(\bar{\omega}_{3}^{2}-\left(\alpha_{2} x_{1}\right)^{2}\right) \bar{\omega}_{2} \\
-\left(\bar{\omega}_{3}^{2}-\left(\alpha_{2} x_{1}\right)^{2}\right) \bar{\omega}_{1} \\
0
\end{array}\right)=\left(\begin{array}{l}
0 \\
0 \\
0
\end{array}\right)
\end{gathered}
$$

Onde, a última implicação é obtida por diferenciação, e usando que $\bar{\omega}_{3} \neq 0$.

Portanto, ou $\bar{\omega}_{1} \equiv \bar{\omega}_{2} \equiv 0$ (de onde segue trivialmente que $\omega$ é constante) ou $\bar{\omega}_{3}^{2}=\alpha_{2}^{2} x_{1}^{2}$, o que não pode ocorrer.

De fato,

$$
\begin{gathered}
\|\dot{\omega}\|^{2}=\sum_{i} \dot{x}_{i}^{2}=\alpha_{2}^{2} x_{1}^{2}\left(x_{2}^{2}+x_{3}^{2}\right)=\alpha_{2}^{2} x_{1}^{2}\left(\|\omega\|^{2}-x_{1}^{2}\right) \\
\|\dot{\omega}\|^{2}=\sum_{i} \dot{\omega}_{i}^{2}=\bar{\omega}_{3}^{2}\left(\bar{\omega}_{1}^{2}+\bar{\omega}_{2}^{2}\right)=\bar{\omega}_{3}^{2}\left(\|\omega\|^{2}-\bar{\omega}_{3}^{2}\right)
\end{gathered}
$$

Assim, se a equação mencionada valesse, teríamos $\alpha_{2}^{2} x_{1}^{2}=\bar{\omega}_{3}^{2}=x_{1}^{2}$, e portanto, deveria valer $\left|\alpha_{2}\right|=1$. Mas isto contraria o item (iv) do lema (3.2.5).

Isto conclui a prova da afirmação, e portanto do item $(i)$.

O item (ii) é mera consequência das definições de $a(t)$ e $I_{\infty}$, o qual foi definido no lema 3.2.4.

Para obter o item (iii), basta observar que a equação (3.32) fornece: $\omega_{\infty} \times I_{\infty} \omega_{\infty}=0$. 


\section{Capítulo 4}

\section{Modelos}

Desde que o último capítulo destinou-se a uma formulação das equações de movimento para corpos elásticos, propõe-se aqui aplicar os resultados a contextos mais específicos, explicitando a geometria e as propriedades internas do sistema.

Naturalmente, esta abordagem permite estabelecer mais resultados, que talvez não valham no caso geral da seção anterior.

Os dois primeiros modelos descritos aqui são apresentados na referência [BL87].

Devido às motivações para este estudo, seria interessante utilizar as equações do teorema (3.2.2) ao caso de corpos rígidos com hastes flexíveis agregadas (sistema isolado).

Isto reflete a situação de um satélite artificial, por exemplo. Além disso, seria um "análogo em dimensão infinita" para o modelo da seção (2.3). A mola com amortecimento seria substituída por uma haste flexível que dissipa energia quando em oscilação. Matematicamente, na descrição do movimento, troca-se a posição da partícula, $x(t) \in \mathbb{R}$, pela deformação da haste: $u(t, \cdot) \in \mathcal{C}(\mathbb{R})$, um espaço de funções.

\subsection{Hastes flexíveis fixas a corpos rígidos}

Nos cálculos e deduções da seção anterior, não foi mencionada a "natureza" da integral. Tudo o que foi feito é válido se tivermos a integral de Riemann (ou considerarmos $d m$ como a medida de Lebesgue em $\mathbb{R}^{3}$ ).

Considere um corpo rígido (ocupando um volume correspondente a um aberto de $\mathbb{R}^{3}$ ) com uma haste flexível de comprimento $1 \mathrm{com}$ uma de suas extremidades fixas a ele.

Considere a haste na posição relaxada e defina como eixo $z$ o eixo fixo ao corpo que contém a haste neste estado. A origem do sistema de coordenadas do corpo é tomada no ponto que conecta a haste ao corpo.

O sistema será considerado livre para se movimentar no espaço, sem interações externas. Ele é representado na figura (4.1).

Pretende-se aplicar o desenvolvimento do capítulo (3) neste contexto. No entanto, a extensão deste corpo não consiste em um aberto. A haste, na posição relaxada, é representada por um intervalo do eixo z.

Seria desejável desenvolver o conteúdo apresentado com hipóteses mais fracas sobre $d m$, e obter as equações de movimento para este corpo como caso particular.

Para os propósitos deste capítulo, no entanto, a postura adotada é reconhecer que as equações finais deduzidas fazem sentido para este corpo, se convenientemente adaptadas.

Denote por $\mathcal{R} \subset \mathbb{R}^{3}$ a região delimitada pela parte rígida do corpo, e $h=\{0\} \times\{0\} \times[0,1]$ a região delimitada pela haste (em repouso). 


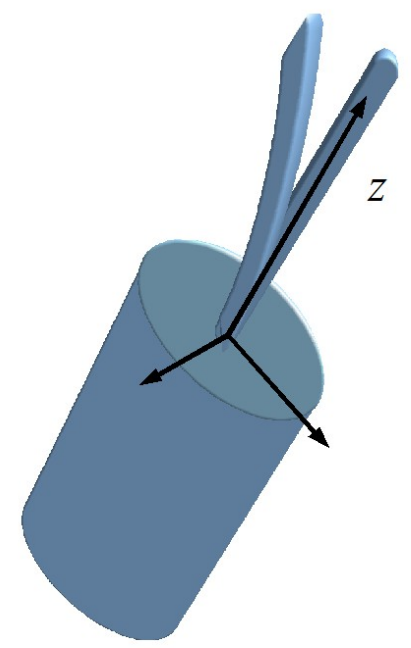

Figura 4.1: Corpo rígido livre com uma haste agregada.

À variável associada à deformação, $u(t, \cdot)$, são impostas as restrições de ser suave $\left(e m \mathbb{R}^{3}\right)$ e:

$$
\left\{\begin{array}{l}
u(t, x, y, z)=(x, y, z), \text { se }(x, y, z) \in \mathcal{R} \\
u(t, x, y, z)=u(t, z), \text { se }(x, y, z) \in h \\
u_{1,2}(t, 0)=\partial_{z} u_{1,2}(t, 0)=\partial_{z}^{2} u_{1,2}(t, 1)=\partial_{z}^{3} u_{1,2}(t, 1)=0, \quad \forall t \\
u_{3}(t, 0)=\partial_{z} u_{3}(t, 1)=0 \quad \forall t
\end{array}\right.
$$

Onde $\left.u\right|_{h}(t, z)=\left(u_{1}, u_{2}, z+u_{3}\right)$.

Assim, o espaço de funções $\mathcal{C}$ citado na seção anterior é o espaço das funções suaves que satisfazem às propriedades acima mencionadas. Note que a identidade, $u(t, \cdot):=I d_{\mathbb{R}^{3}}$, claramente está neste espaço. Além disso, se $\eta \in \mathcal{V}$, trivialmente, estas propriedades também são satisfeitas.

A energia potencial de deformação está associada somente à haste, desde que na parte rígida a energia é puramente cinética. Assumindo que a haste possui seção transversal uniforme, esta energia é dada por:

$$
V(u, Y, y)=V(u):=\frac{1}{2} \int_{0}^{1} \mu_{1}\left(\partial_{z z} u_{1}\right)^{2}+\mu_{2}\left(\partial_{z z} u_{2}\right)^{2}+\mu_{3}\left(\partial_{z} u_{3}\right)^{2} d z
$$

Também pode-se trabalhar sem a referida hipótese. A situação seria como se houvessem vínculos internos, talvez alterando até a forma das equações de movimento. Este tipo de tratamento é também exposto em [BL91].

Esta energia também é chamada de energia de deformação e, juntamente com as condições de contorno da equação (4.1), advém da teoria de elasticidade (unidimensional, para hastes). Está se supondo que o material satisfaz o que se chama de lei de Hooke linear, por isso a dependência quadrática em cada termo (é análoga à energia potencial de uma mola).

Observe que $V$ depende apenas da primeira derivada da terceira componente, $u_{3}$, da deformação. Normalmente, os coeficientes satisfazem: $\mu_{3} \gg \mu_{1}, \mu_{2}$. Isto é reflexo da maior resistência da haste em vibrar na direção de seu eixo.

De forma análoga ao caso da mola, a função dissipação é parecida com a potencial, trocando as posições pelas velocidades dos pontos materiais. Há também a ideia de "dissipação linear".

$$
\mathcal{D}:=\frac{1}{2} \int_{0}^{1} k_{1}\left(\partial_{z z} \dot{u}_{1}\right)^{2}+k_{2}\left(\partial_{z z} \dot{u}_{2}\right)^{2}+k_{3}\left(\partial_{z} \dot{u}_{3}\right)^{2} d z
$$

Onde $\dot{u}$ denota $\partial_{t} u$. 
Observe que o potencial de deformação é invariante pela mudança do referencial inercial para o corpo. O mesmo não ocorre com a função dissipação (assim como a energia cinética), pois, no referencial inercial a deformação é $U=Y u+y \Rightarrow U_{t}=Y\left(\Omega u+Y^{T} \dot{y}\right)$.

Para estas funções, temos:

$$
\frac{\partial V}{\partial Y}=\frac{\partial V}{\partial y}=\frac{\partial V}{\partial \dot{Y}}=\frac{\partial V}{\partial \dot{y}}=\frac{\partial \mathcal{D}}{\partial Y}=\frac{\partial \mathcal{D}}{\partial y}=\frac{\partial \mathcal{D}}{\partial \dot{Y}}=\frac{\partial \mathcal{D}}{\partial \dot{y}}=0 .
$$

Para calcular $\delta V / \delta u$, considere, $\eta \in \mathcal{V}$ :

$$
\delta_{u} V(\eta)=\frac{1}{2} \frac{d}{d \epsilon} \int_{0}^{1} \mu_{1}\left(\partial_{z z}\left(\phi_{\epsilon}\right)_{1}\right)^{2}+\mu_{2}\left(\partial_{z z}\left(\phi_{\epsilon}\right)_{2}\right)^{2}+\left.\mu_{3}\left(\partial_{z z}\left(\phi_{\epsilon}\right)_{3}\right)^{2} d z\right|_{\epsilon=0}=\Delta .
$$

Onde

$$
\begin{gathered}
\Delta=\int_{0}^{1} \mu_{1} u_{1}^{\prime \prime} \eta_{1}^{\prime \prime}+\mu_{2} u_{2}^{\prime \prime} \eta_{2}^{\prime \prime}+\mu_{3} u_{3}^{\prime} \eta_{3}^{\prime} d z= \\
=\sum_{i=1,2} \mu_{i}\left(\left.u_{i}^{\prime \prime} \eta_{i}^{\prime}\right|_{0} ^{1}-\left.u_{i}^{\prime \prime \prime} \eta_{i}\right|_{0} ^{1}+\int_{0}^{1} \eta_{i} \partial_{z}^{4} u_{i} d z\right)+\mu_{3}\left(\left.u_{3}^{\prime} \eta_{3}\right|_{0} ^{1}-\int_{0}^{1} \eta_{3} \partial_{z}^{2} u_{3} d z\right) .
\end{gathered}
$$

A igualdade foi obtida por sucessivas integrais por partes. Assim, por tanto $u_{i}$ quanto $\eta_{i}$ satisfazerem as condições de contorno, e por um cálculo análogo para o segundo termo, conclui-se que:

$$
\delta_{u} V(\eta)=\int_{0}^{1} \mu_{1} \eta_{1} \partial_{z}^{4} u_{1}+\mu_{2} \eta_{2} \partial_{z}^{4} u_{2}-\mu_{3} \eta_{3} \partial_{z}^{2} u_{3} d z .
$$

Assim, fica claro, da definição da derivada que:

$$
\frac{\delta V}{\delta u}=\left(\mu_{1} \partial_{z}^{4} u_{1}, \mu_{2} \partial_{z}^{4} u_{2},-\mu_{3} \partial_{z}^{2} u_{3}\right)=: \mu\left(\partial_{z}^{4}, \partial_{z}^{4},-\partial_{z}^{2}\right) u=: \mu \partial u .
$$

De forma completamente análoga, concluímos que:

$$
\frac{\delta \mathcal{D}}{\delta v}=\left(k_{1} \partial_{z}^{4} v_{1}, k_{2} \partial_{z}^{4} v_{2},-k_{3} \partial_{z}^{2} v_{3}\right)=: k\left(\partial_{z}^{4}, \partial_{z}^{4},-\partial_{z}^{2}\right) v=: k \partial u_{t}
$$

Onde as últimas igualdades são apenas as definições destes símbolos.

Observe que, como as condições de contorno nas equações (4.1) valem para todo $t$, valem as mesmas condições para suas derivadas temporais. Portanto, o cálculo aqui esboçado pode ser reproduzido sem alterações.

Teorema 4.1.1. Para este sistema, as equações de movimento são:

$$
\begin{gathered}
I \dot{\omega}+\omega \times I \omega+m_{b} \bar{c} \times Y^{-1} \ddot{y}+\int_{0}^{1} u \times\left(D^{2} u+Y^{-1} \ddot{y}\right) d z=0 \\
D^{2} u+\mu \partial u+k \partial u_{t}+Y^{-1} \ddot{y}=0 \\
m_{b} \frac{d^{2}}{d t^{2}}(y+Y \bar{c})+\int_{0}^{1}\left(Y D^{2} u+\ddot{y}\right) d z=0
\end{gathered}
$$

Onde $m_{b}$, I e $\bar{c}:=\left(\int_{\mathcal{R}} u d m^{\prime}\right) / m_{b}$ são a massa, o operador de inércia e o centro de massa da parte rígida, respectivamente. E o operador D foi definido no corolário (4.4.1).

Demonstração. Neste caso,

$$
\int_{\mathcal{B}} u \times\left(D^{2} u+Y^{-1} \ddot{y}\right) d m=\int_{\mathcal{R}} u \times\left(D^{2} u+Y^{-1} \ddot{y}\right) d m+\int_{h} u \times\left(D^{2} u+Y^{-1} \ddot{y}\right) d z=
$$




$$
=\int_{\mathcal{R}} r \times\left(\dot{\omega} \times r+\omega \times(\omega \times r)+Y^{-1} \ddot{y}\right) d m+\int_{0}^{1} u \times\left(D^{2} u+Y^{-1} \ddot{y}\right) d z .
$$

Onde $r(x, y, z):=(x, y, z)$.

Mas,

$$
\int_{\mathcal{R}} r \times(r \times \dot{\omega}) d m^{\prime}=-I \dot{\omega} .
$$

$\mathrm{E}, r \times(\omega \times(\omega \times r))+(\omega \times r) \times(r \times \omega)+\omega \times((\omega \times r) \times r)=0$.

$$
\Rightarrow \int_{\mathcal{R}} r \times(\omega \times(\omega \times r)) d m^{\prime}=-\omega \times \int_{\mathcal{R}} r \times(r \times \omega) d m^{\prime}=\omega \times I \omega .
$$

Portanto, da equação (3.27), concluímos (4.4).

A equação (4.5) é obtida diretamente das equações (3.28), (4.12) e (4.13).

Para a terceira equação, calculemos,

$$
\int_{\mathcal{B}} D^{2} u+Y^{-1} \ddot{y} d m=m_{b} \dot{\omega} \times \bar{c}+m_{b} \omega \times(\omega \times \bar{c})+m_{b} Y^{-1} \ddot{y}+\int_{0}^{1} D^{2} u+Y^{-1} \ddot{y} d z .
$$

Logo, da equação (3.29),

$$
m_{b}(Y \dot{\omega} \times \bar{c}+Y \omega \times(\omega \times \bar{c})+\ddot{y})+\int_{0}^{1} Y D^{2} u+\ddot{y} d z=0 .
$$

Além disso,

$$
\frac{d^{2}}{d t^{2}}(Y \bar{c})=\frac{d}{d t}(Y \Omega \bar{c})=Y \Omega^{2} \bar{c}+Y \dot{\Omega} \bar{c}=Y \omega \times(\omega \times \bar{c})+Y \dot{\omega} \times \bar{c} .
$$

Daí é concluída (4.6).

Note que qualquer solução deste sistema é livre de torque. Assim, pelas considerações feitas na seção (3.2), constata-se que, com a escolha correta do referencial inercial:

$$
m y+Y \int_{0}^{1}\left(u+m_{b} \bar{c}\right) d z=0 \Rightarrow y=-Y C
$$

Onde $m=m_{b}+1$ é a massa total do sistema e, novamente, $C$ é o centro de massa do sistema completo.

Note que a conservação do momento angular também é consequência da propriedade citada.

Neste modelo já é possível observar uma relação mais interessante entre a variação de energia e a função $\mathcal{D}$. Pela equação (3.38), temos:

$$
\dot{E}=-\left\langle u_{t}, \frac{\delta \mathcal{D}}{\delta v}\right\rangle=-\int_{0}^{1} k_{1} v_{1} \partial_{z}^{4} v_{1}+k_{2} v_{2} \partial_{z}^{4} v_{2}-k_{3} v_{3} \partial_{z}^{2} v_{3} d m
$$

Assim, observando novamente as condições de contorno e fazendo as mesmas passagens do cálculo do $\Delta$, concluímos que:

$$
\dot{E}=-\int_{0}^{1} k_{1}\left(\partial_{z}^{2} v_{1}\right)^{2}+k_{2}\left(\partial_{z}^{2} v_{2}\right)^{2}+k_{3}\left(\partial_{z} v_{3}\right)^{2} d m=-2 \mathcal{D}\left(u_{t}\right) \leq 0 .
$$

Esse sistema realmente tem a propriedade de ser dissipativo. Observe que a diminuição da energia é devida somente ao movimento da haste (relativo à parte rígida).

Outra propriedade interessante é que a energia deixa de diminuir se e somente se $u_{t} \equiv 0$ $(\Leftrightarrow u(t, z) \equiv u(z))$. 
De fato,

$$
\dot{E}=0 \Leftrightarrow\left\{\begin{array}{l}
\partial_{z}^{2} \partial_{t} u_{1}(t, z)=0, \\
\partial_{z}^{2} \partial_{t} u_{2}(t, z)=0, \quad \forall t, z \\
\partial_{z} \partial_{t} u_{3}(t, z)=0,
\end{array}\right.
$$

Assim, $\partial_{z} v_{1}(t, z)=c_{1}(t)$. E, das condições de contorno, $c_{1}(t)=\partial_{z} v_{1}(t, 0)=0, \forall t$. Logo, $v_{1}(t, z)=c_{2}(t) \Rightarrow c_{2}(t)=v_{1}(t, 0)=0, \forall t \Rightarrow v_{1} \equiv 0$. Analogamente para $u_{2}$.

Para $\partial_{z} \partial_{t} u_{3}(t, z)=0 \Rightarrow v_{3}(t, z)=c_{1}(t) \Rightarrow c_{1}(t)=v_{3}(t, 0)=0 \Rightarrow u_{3} \equiv 0$. A recíproca é trivial.

Observe que, mesmo nesse modelo específico, o conhecimento sobre as soluções de dissipação nula restringiu-se apenas ao que foi obtido na seção (3.2). Ou seja, de que a rotação é dada em torno de um eixo fixo no espaço cuja direção é auto espaço do operador de inércia $I_{\infty}$ apresentado.

No entanto, seria interessante investigar se há alguma rotação de equilíbrio que seja "estável" ou que atraia a "maioria" das soluções como imaginado na exposição da seção (2.2). Visto que a mesma ocorreu, de certa forma, no modelo da seção (2.3).

Com este intuito, observemos o que ocorre com as soluções estacionárias.

Para este caso, em vista do teorema (3.2.3), a velocidade angular é constante, $\omega_{\infty}$. Logo, a equação (4.5) fica:

$$
\omega_{\infty} \times\left(\omega_{\infty} \times u\right)+Y^{T} \ddot{y}=-\mu \partial u .
$$

Mas, desde que a posição do centro de massa é constante no tempo, $Y^{T} \ddot{y}=-D^{2} C=-\omega_{\infty} \times$ $\left(\omega_{\infty} \times C\right)$.

Logo, sendo $\Omega_{\infty}=S^{-1}\left(\omega_{\infty}\right)$,

$$
\mu \partial u=-\Omega_{\infty}^{2}(u-C) .
$$

Que é essencialmente uma equação diferencial ordinária de quarta ordem com as condições de contorno (4.1) e, adicionalmente, aquelas impostas pela equação (4.4), que é do tipo "integrodiferencial".

Não é objetivo deste trabalho tratar esta equação. Assim como não foi feito pelos autores da referência.

Note que não foram encontrados vestígios do comportamento assintótico natural acerca deste problema, e nem foi possível apontar alguma característica que o impeça de ocorrer.

\subsection{Caso de rotação restrita a um plano}

A fim de facilitar a análise citada no final da seção anterior, considere o mesmo problema com a adição de certas restrições.

Para diminuir o número de variáveis, restrinja a liberdade de rotação exigindo que o corpo seja chato, vinculado a girar somente sobre um plano fixo no referencial inercial, sem atrito, paralelo ao $(x, y)$. Suponha que a haste somente oscile ao longo de um plano, digamos $(y, z)$ (que é fixo ao corpo), diminuindo também as coordenadas de deformação da haste para só uma $\left(u_{2}\right)$.

Suponha que a parte rígida seja um disco, simétrico com respeito ao eixo $z$, ao longo do qual fica a haste na posição relaxada, de forma que a origem do referencial móvel permaneça fixa durante o movimento. Isto elimina a variável $y$.

A figura (4.2) ilustra a situação.

A imposição destes vínculos adicionais modificam os espaços onde estão definidas as coordenadas. Consequentemente mudando a dedução das equações de movimento.

Aqui, a variável associada à rotação do corpo está em $S O(2)$, o grupo das matrizes de ordem dois, ortogonais e de determinante positivo, o qual pode ser naturalmente mergulhado em $S O(3)$. Daí, pode-se calcular a projeção ortogonal dos espaços tangentes e usar os resultados do capítulo 3.

Esta não é uma análise elaborada, a qual os autores da referência não mencionam. Talvez porque as equações de movimento sejam exatamente as mesmas da seção (4.1), com a restrição das variáveis que o presente modelo impõe. 


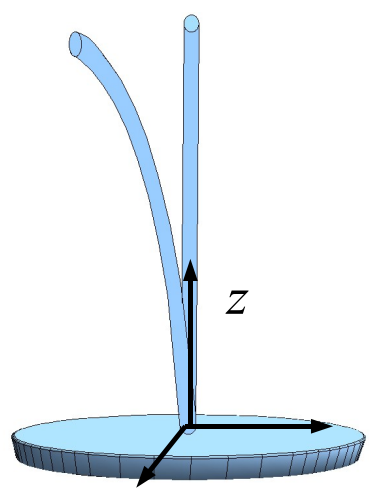

Figura 4.2: Haste elástica anexa a disco rígido submetido a movimento plano .

Desta forma, a matriz de rotação $Y$ somente pode ser da forma:

$$
Y(t)=\left(\begin{array}{ccc}
\cos \theta(t) & -\operatorname{sen} \theta(t) & 0 \\
\operatorname{sen} \theta(t) & \cos \theta(t) & 0 \\
0 & 0 & 1
\end{array}\right) \Rightarrow \Omega=Y^{T} \dot{Y}(t)=\dot{\theta}\left(\begin{array}{ccc}
0 & -1 & 0 \\
1 & 0 & 0 \\
0 & 0 & 0
\end{array}\right) .
$$

Portanto, $\omega(t)=(0,0, \dot{\theta})=:(0,0, \omega)$ (este é um abuso de notação que não causará enganos).

Pelas hipóteses, a deformação é a identidade sobre o disco. E sobre a haste, é da forma: $u(t, z)=$ $\left(0, u_{2}(t, z), u_{3}(t, z)+z\right)$.

Assim, da equação (3.24), o vetor $Y a(t)$ é conservado.

Calculemos a terceira componente $a$ :

$$
\begin{aligned}
<a(t), \hat{z}> & =\left\langle\int_{\mathcal{R}} r \times(r \times \omega) d m+\int_{0}^{1}\left(0, u_{2}, u_{3}+z\right) \times\left(-\omega u_{2}, \partial_{t} u_{2}, \partial_{t} u_{3}\right) d z, \hat{z}\right\rangle= \\
& =\int_{D} \omega\left(x^{2}+y^{2}\right) \rho d x d y+\omega \int_{0}^{1} u_{2}^{2} d z=\omega\left(I+\int_{0}^{1} u^{2} d z\right) .
\end{aligned}
$$

Onde, novamente por um abuso de notação, $u:=u_{2}$, e $I:=\int_{D}\left(x^{2}+y^{2}\right) \rho d x d y$ é o momento de inércia do disco com respeito ao eixo $z$.

Logo, desde que a terceira componente de $a$ é igual à terceira componente de $Y a$, decorre que

$$
\omega\left(I+\int_{0}^{1} u^{2} d z\right)=M=\text { constante. }
$$

Observação 4.2.1. Em vista da forma que a matriz de rotação, $Y$, adquire neste modelo, está sendo suposto que as derivadas de $V$ e $\mathcal{D}$ que aparecem na equação (3.24) têm terceira componente nula. Isto é imposto, de maneira informal aqui, devido a um argumento intuitivo.

Como a haste está oscilando da maneira descrita, ela exerce um torque total não nulo que necessariamente está no plano $(x, y)$. Portanto, a priori, poderíamos "inferir" somente a conservação da componente $z$ do momento angular.

Para uma justificativa mais clara, consultar o apêndice B.

Quanto à equação (3.25), sua segunda componente se reduz a:

$$
u_{t t}+\mu u_{z z z z}+k u_{z z z z t}=\omega^{2} u .
$$

Onde $\mu:=\mu_{2}$ e $k:=k_{2}$.

A terceira componente da mesma equação fornece que $\partial_{t}^{2} u_{3}=0$. Assim, pelas condições de contorno, $u_{3} \equiv 0$. 
Desta forma, se uma solução é estacionária, ela é solução do seguinte problema (não linear):

$$
\begin{gathered}
\mu u_{z z z z}-\omega^{2} u=0 \\
\omega\left(I+\int_{0}^{1} u^{2} d z\right)=M \\
u(t, 0)=u_{z}(t, 0)=u_{z z}(t, 1)=u_{z z z}(t, 1)=0
\end{gathered}
$$

Observação 4.2.2. Os autores da referência indicada afirmam que as soluçôes deste problema são os pontos críticos da seguinte função energia:

$$
E(\omega, u, v)=\frac{1}{2} I \omega^{2}+\frac{1}{2} \int_{0}^{1} v^{2}+u_{z z}^{2}+\omega^{2} u^{2} d z
$$

quando restrita à condição de momento angular $M$ constante.

O motivo de ter sido citado neste trabalho, é sua interessante compatibilidade com a discussão da seção (2.2), onde foi sugerido que a rotação estacionária "estável" estaria em um mínimo de energia sobre o conjunto de momento angular constante.

Os autores ainda afirmam que o operador diferencial $\partial^{4} / \partial z^{4}$, com as condições de contorno dadas pela terceira equação do sistema (4.11), possui uma sequência de autofunções não triviais, $u_{1}(z), u_{2}(z), \cdots$.

Associada a esta sequência de soluções, temos uma sequência de velocidades angulares correspondentes (autovalores): $0<\omega_{1}<\omega_{2}<\cdots$. E ainda, $\lim _{i \rightarrow \infty} \omega_{i}=+\infty$. Cada $\omega_{i}$ deve satisfazer:

$$
\omega_{i}=\frac{M}{I+\int_{0}^{1} u_{i}(z)^{2} d z} .
$$

Estas velocidades angulares desempenham um papel central no seguinte resultado, o principal da referência [BL87], que será somente enunciado aqui devido à dificuldade encontrada em sua demonstração.

Teorema 4.2.1. Suponha que haja dissipação $(k>0)$.

Então, as mencionadas velocidades angulares são tais que, para o momento angular no intervalo: $M \in\left(I \omega_{j}, I \omega_{j+1}\right)$, o problema (4.14) (com as condiçôes de contorno) possui $j$ soluçôes estacionárias, não triviais e distintas: $u(t, z)=u_{i}(z), 1 \leq i \leq j$, além da solução trivial.

Além disso, considere a norma:

$$
\left\|\left(u, u_{t}\right)\right\|=\|(u, v)\|=\left(\int_{0}^{1} u^{2}+v^{2} d z\right)^{\frac{1}{2}} .
$$

Então, se $j \geq 1$, a solução com menor velocidade angular $\left(u_{1}\right)$ é "dinamicamente estável", isto é, qualquer solução com condição inicial próxima a ela, mantém-se próxima para todo tempo, nesta norma. Caso $M<I \omega_{1}$, a única solução estacionária é a trivial, que é dinamicamente estável. 


\subsection{Aplicação ao corpo rígido com oscilador}

Como mencionado na seção (2.3), as equações de movimento deduzidas para o corpo rígido com oscilador também decorrem de um tratamento Lagrangiano.

Infelizmente, aquele contexto compreende objetos que são tratados em mecânica de partículas e em mecânica do contínuo, o corpo rígido e o sistema massa-mola. Para aplicar o formalismo Lagrangiano, há novamente a dificuldade citada na seção (4.1). A medida de massa $d m$, somente como medida de volume sobre $\mathbb{R}^{3}$, parece não ser suficiente para traduzir matematicamente $o$ problema.

Assim, a postura aqui adotada é partir da energia cinética dada pela equação (3.2), adaptando-a de forma natural (aditivamente) ao problema.

Note que o referencial do corpo é adotado sobre o centro de massa, o qual sabemos que está em repouso, o que elimina a coordenada $y$. A priori, dever-se-ia incluir esta coordenada, deduzir as equações de movimento, e mostrar este fato. Mas isto será assumido.

A energia cinética é (por definição):

$$
T(\dot{Y}, \dot{x}, Y, x)=\frac{1}{2} \int_{\mathcal{R}}\|\Omega r\|^{2} d m+m_{p}\|x \Omega \hat{x}+\dot{x} \hat{x}\|^{2} .
$$

Onde o primeiro termo é a energia cinética do corpo rígido e o segundo das partículas, como natural ( $m_{p}$ é a massa das partículas).

Como o corpo rígido não se deforma, a energia potencial e a função de Rayleigh reduzem-se apenas às mesmas do sistema massa-mola:

$$
V(x)=k\left(x-x_{0}\right)^{2}, \quad \mathcal{D}(\dot{x})=c \dot{x}^{2} .
$$

Para calcular as derivadas da energia cinética com respeito às coordenadas de rotação, o procedimento é uma cópia da seção (4.2). Temos: $T=T_{1}+T_{2}$, com $T_{i}(\dot{Y}, Y)=K_{i}\left(Y^{T} \dot{Y}\right)=K_{i}(\Omega)$.

Da mesma forma,

$$
\begin{gathered}
\left(\frac{\partial K_{1}}{\partial \Omega}\right)_{a}=\int_{\mathcal{R}}\left((\Omega r) r^{T}\right)_{a} d m=\frac{1}{2} \int_{\mathcal{R}} r \times(\omega \times r) d m=\frac{1}{2} I \omega . \\
\left(\frac{\partial K_{2}}{\partial \Omega}\right)_{a}=2 m_{p}\left((x \Omega \hat{x}+\dot{x} \hat{x})(x \hat{x})^{T}\right)_{a}=m_{p}(x \hat{x}) \times(x \omega \times \hat{x}+\dot{x} \hat{x})=m_{p} x^{2} \hat{x} \times(\omega \times \hat{x}) .
\end{gathered}
$$

Portanto, observando que este sistema é "livre de torque", da equação (3.21) decorre:

$$
\frac{d}{d t}\left(I \omega+2 m_{p} x^{2} \hat{x} \times(\omega \times \hat{x})\right)+\omega \times\left(I \omega+2 m_{p} x^{2} \hat{x} \times(\omega \times \hat{x})\right)=0 .
$$

Note que esta equação é equivalente à primeira equação do sistema (2.4), desde que $L=I \omega+$ $2 m_{p} x^{2} \hat{x} \times(\omega \times \hat{x})$.

Por outro lado,

$$
\frac{\partial T}{\partial \dot{x}}=2 m_{p}<x \Omega \hat{x}+\dot{x} \hat{x}, \hat{x}>\Rightarrow \frac{d}{d t}\left(\frac{\partial T}{\partial \dot{x}}\right)=2 m_{p}<\dot{x} \omega \times \hat{x}+x \dot{\omega} \times \hat{x}+\ddot{x} \hat{x}, \hat{x}>.
$$

$\frac{\partial T}{\partial x}=2 m_{p}<x \Omega \hat{x}+\dot{x} \hat{x}, \Omega \hat{x}>=-2 m_{p}<x \Omega^{2} \hat{x}+\dot{x} \Omega \hat{x}, \hat{x}>=-2 m_{p}<x \omega \times(\omega \times \hat{x})+\dot{x} \omega \times \hat{x}, \hat{x}>$.

Logo, a equação de movimento é:

$$
m_{p}<\ddot{x} \hat{x}+2 \dot{x} \omega \times \hat{x}+x \dot{\omega} \times \hat{x}+x \omega \times(\omega \times \hat{x}), \hat{x}>=-k\left(x-x_{0}\right)-c \dot{x} .
$$

E esta é justamente a segunda equação do sistema (2.4). 
Pode-se calcular a energia mecânica do sistema, como definida na equação (3.36).

$$
\begin{gathered}
\left\langle\frac{\partial T}{\partial \dot{Y}}, \dot{Y}\right\rangle=\left\langle\frac{\partial K}{\partial \Omega}, Y^{T} \dot{Y}\right\rangle=\int_{\mathcal{R}}<\Omega r, Y^{T} \dot{Y} r>d m+2 m_{p}<x \Omega \hat{x}+\dot{x} \hat{x}, x Y^{T} \dot{Y} \hat{x}>= \\
=\int_{\mathcal{R}}\|\omega \times r\|^{2} d m+2 m_{p}<x \Omega \hat{x}+\dot{x} \hat{x}, x \Omega \hat{x}> \\
\frac{\partial T}{\partial \dot{x}} \dot{x}=2 m_{p}<\dot{x} \hat{x}, x \Omega \hat{x}+\dot{x} \hat{x}>.
\end{gathered}
$$

Logo,

$$
\begin{gathered}
E=\frac{1}{2} \int_{\mathcal{R}}<r \times(\omega \times r), \omega>d m+m_{p}\|x \omega \times \hat{x}+\dot{x} \hat{x}\|^{2}+k\left(x-x_{0}\right)^{2} \\
\Rightarrow E=\frac{1}{2}<I \omega, \omega>+m_{p}\|x \omega \times \hat{x}+\dot{x} \hat{x}\|^{2}+k\left(x-x_{0}\right)^{2} .
\end{gathered}
$$

Que é exatamente a energia definida em (2.5). O que confirma sua compatibilidade com a definição de energia apresentada em (3.36).

\subsection{Elasticidade tridimensional}

Uma situação física interessante que não foi explorada até agora é o caso de um corpo sólido que não tenha alguma parte rígida. Um corpo que não sofra ação de forças externas e que seja deformável.

É razoável imaginar que qualquer objeto real seria melhor modelado com este princípio, já que a rigidez perfeita é uma idealização.

No entanto, pensando desta forma, surgem problemas parecidos com aquele mencionado na seção (2.1). É a dificuldade de modelar uma estrutura extremamente complexa de forma a não tornar o problema matematicamente intratável e, ao mesmo tempo, não trivializá-lo ou desprovir de significado físico.

Em geral, objetos sólidos possuem densidade de massa não homogênea, o que usualmente indica diferente resistência à deformação ao longo de sua extensão. Além de possuir articulações, que se movimentam e dissipam energia de forma completamente distinta de outras partes.

Nesta seção, os objetos de estudo são corpos que possuem elasticidade tridimensional, ou seja, que se deformam (dissipando energia) em todas as direções do espaço, não possuindo parte rígida.

Há dificuldades conceituais que estas propriedades envolvem. Elas dificultam principalmente a interpretação física na formulação e nos resultados obtidos.

Não é mais intuitivamente claro qual seria o ponto do corpo tomado como origem do referencial móvel. Neste problema, é difícil visualizar uma configuração relaxada que o acompanha. Desde que o sistema está isolado, é razoável assumir que seu centro de massa permanece em repouso (em relação a um referencial inercial) durante o movimento, vistos os resultados das seções anteriores, e pelas funções potencial e de dissipação apresentadas a seguir. Assim, tome o ponto de referência do corpo no centro de massa, eliminando a coordenada de movimento $y \equiv 0$.

A segunda dificuldade está na definição dos eixos do referencial do corpo. Dada a posição inicial, imagine a configuração relaxada do corpo naquela posição, e uma base ortonormal de autovetores do operador de inércia (relaxado). A base móvel será determinada pela imagem desta base pela matriz $Y(t)$, solução das equações de movimento deduzidas aqui.

Outras restrições que são impostas, essenciais, são a linearidade e a homogeneidade da deformação e da dissipação. Ou seja, que elas se comportem como na mola e na haste, cujas forças eram lineares no deslocamento e na velocidade (respectivamente), agora nas direções $x, y$ e $z$.

Este conceito análogo a força, sobre cada elemento do corpo, é desempenhado através dos chamados tensores de tensão e de tensão viscosa. Exigindo sua linearidade, obtemos as funções 
potencial e de dissipação (consultar por exemplo [LL86]):

$$
\begin{aligned}
V(Y, u, y) & :=\int_{\mathcal{B}}\left[\frac{\mu}{2}\left(\left(\partial_{i} u_{k}\right)^{2}+\partial_{k} u_{i} \partial_{i} u_{k}\right)+\left(\frac{K}{2}-\frac{\mu}{3}\right)\left(\partial_{l} u_{l}\right)^{2}\right] d V, \\
\mathcal{D}(\dot{Y}, v, \dot{y}) & :=\int_{\mathcal{B}}\left[\frac{\eta}{2}\left(\left(\partial_{i} v_{k}\right)^{2}+\partial_{k} v_{i} \partial_{i} v_{k}\right)+\left(\frac{\zeta}{2}-\frac{\eta}{3}\right)\left(\partial_{l} v_{l}\right)^{2}\right] d V,
\end{aligned}
$$

onde $\mu, K, \eta, \zeta$ são constantes e foi feito uso da notação de soma. Uma exigência "técnica" sobre o volume do corpo, $\mathcal{B} \subset \mathbb{R}^{3}$, é que ele seja aberto com bordo suave, de modo a poder aplicar o teorema de Stokes.

Defina, os tensores de tensão e de tensão viscosa, respectivamente, através de suas componentes:

$$
\begin{aligned}
\sigma_{i k} & :=\left(\left(K-\frac{2 \mu}{3}\right) \nabla u\right) \delta_{i k}+\mu\left(\partial_{i} u_{k}+\partial_{k} u_{i}\right) \\
\tilde{\sigma}_{i k} & :=\left(\left(\zeta-\frac{2 \eta}{3}\right) \nabla v\right) \delta_{i k}+\eta\left(\partial_{i} v_{k}+\partial_{k} v_{i}\right)
\end{aligned}
$$

Para o significado físico destes objetos, consultar a referência [LL86] (parágrafo 4 para o primeiro, e parágrafo 34 para o segundo).

Exigiremos tais tensores tenham componente normal nula sobre o bordo. Esta é uma condição típica, que será exigida valer para todos os difeomorfismos do espaço $\mathcal{C}$. Logo,

$$
\begin{gathered}
(\sigma n(x))_{i}=\left(\left(K-\frac{2 \mu}{3}\right) \nabla u(t, x)\right) n_{i}(x)+\mu\left(\partial_{i} u_{k}(t, x)+\partial_{k} u_{i}(t, x)\right) n_{k}(x)=0 \\
(\tilde{\sigma} n(x))_{i}=\left(\left(\zeta-\frac{2 \eta}{3}\right) \nabla v(t, x)\right) n_{i}(x)+\eta\left(\partial_{i} v_{k}(t, x)+\partial_{k} v_{i}(t, x)\right) n_{k}(x)=0
\end{gathered}
$$

Para todo $x \in \partial \mathcal{B}$, e para todo $t$. Onde $n(x):=\left(n_{1}(x), n_{2}(x), n_{3}(x)\right)$ é o vetor normal (exterior) a $\partial \mathcal{B}$.

Desta forma, dados $u \in \mathcal{C}$ e $h \in \mathcal{V}$, obtemos:

$$
\begin{gathered}
\delta_{u} V(h)=\left.\frac{d}{d \epsilon} \int_{\mathcal{B}}\left[\frac{\mu}{2}\left(\left(\partial_{i}\left(\phi_{\epsilon}\right)_{k}\right)^{2}+\left(\partial_{k}\left(\phi_{\epsilon}\right)_{i}\right)\left(\partial_{i}\left(\phi_{\epsilon}\right)_{k}\right)\right)+\left(\frac{K}{2}-\frac{\mu}{3}\right)\left(\partial_{l}\left(\phi_{\epsilon}\right)_{l}\right)^{2}\right] d V\right|_{\epsilon=0} \\
=\int_{\mathcal{B}}\left[\mu\left(\partial_{i} u_{k} \partial_{i} h_{k}+\partial_{i} u_{k} \partial_{k} h_{i}\right)+\left(K-\frac{2 \mu}{3}\right) \partial_{l} u_{l} \partial_{j} h_{j}\right] d V \\
=\int_{\mathcal{B}}\left[\mu\left(<\nabla u_{i}, \nabla h_{i}>+<\nabla h_{i}, \partial_{i} u>\right)+\left(K-\frac{2 \mu}{3}\right)(\nabla u)(\nabla h)\right] d V .
\end{gathered}
$$

Mas, por uma identidade vetorial elementar e pelo teorema da divergência, para todos $\alpha$ campo escalar e $w$ campo vetorial,

$$
\int_{\mathcal{B}}<\nabla \alpha, w>+\alpha \nabla w d V=\int_{\mathcal{B}} \nabla(\alpha w) d V=\int_{\partial \mathcal{B}} \alpha<w, n>d S .
$$

Logo, fazendo $\alpha=h_{i}$ e $w=\sigma_{i}$, a $i$-ésima coluna do tensor $\sigma$, decorre:

$$
\begin{aligned}
<\sigma_{i}, \nabla h_{i}> & =\sigma_{i k} \partial_{k} h_{i}=\left(\left(K-\frac{2 \mu}{3}\right) \nabla u\right) \partial_{i} h_{i}+\mu\left(<\partial_{i} u, \nabla h_{i}>+<\nabla u_{i}, \nabla h_{i}>\right) \\
& =\left(K-\frac{2 \mu}{3}\right)(\nabla u)(\nabla h)+\mu\left(<\partial_{i} u, \nabla h_{i}>+<\nabla u_{i}, \nabla h_{i}>\right) .
\end{aligned}
$$

Mas, da identidade citada:

$$
\int_{\mathcal{B}}<\nabla h_{i}, \sigma_{i}>+h_{i} \nabla \sigma_{i} d V=\int_{\partial \mathcal{B}} h_{i}<\sigma_{i}, n>d S=0,
$$

pela condição de contorno (4.17). 
Mas constata-se que $\nabla \sigma_{i}=\partial_{k} \sigma_{i k}=\left(K+\frac{\mu}{3}\right) \partial_{i}(\nabla u)+\mu \Delta u_{i}$. Logo,

$$
h_{i} \nabla \sigma_{i}=\left(K+\frac{\mu}{3}\right)<\nabla(\nabla u), h>+\mu<\Delta u, h>.
$$

Portanto,

$$
\begin{gathered}
\delta_{u} V(h)=\int_{\mathcal{B}}<\sigma_{i}, \nabla h_{i}>d V \\
=-\int_{\mathcal{B}}\left\langle\left(K+\frac{\mu}{3}\right) \nabla(\nabla u)+\mu \Delta u, h\right\rangle d V, \quad \forall h \in \mathcal{V} .
\end{gathered}
$$

Desta forma, concluímos que:

$$
\frac{\delta V}{\delta u}=-\left(K+\frac{\mu}{3}\right) \nabla(\nabla u)-\mu \Delta u
$$

analogamente,

$$
\frac{\delta \mathcal{D}}{\delta v}=-\left(\zeta+\frac{\eta}{3}\right) \nabla(\nabla v)-\zeta \Delta v
$$

Note que (consultar, por exemplo referência [LL87], parágrafo 15) esta última função é exatamente a que ocorre na equação de Navier-Stokes para fluidos isotrópicos, com viscosidade. Logo, podemos resumir estes cálculos em mais um corolário do teorema (3.2.2). Observando que o sistema é livre de torque, temos:

Teorema 4.4.1. As equaçôes de movimento para um corpo elástico tridimensional reduzem-se a:

$$
\begin{gathered}
M=Y \int_{\mathcal{B}} u \times D u d m=\text { constante } \Leftrightarrow \int_{\mathcal{B}} u \times D^{2} u d m=0 \\
D^{2} u=u_{t t}+\dot{\omega} \times u+2 \omega \times u_{t}+\omega \times(\omega \times u)=\left(K+\frac{\mu}{3}\right) \nabla(\nabla u)+\mu \Delta u+\left(\zeta+\frac{\eta}{3}\right) \nabla\left(\nabla u_{t}\right)+\zeta \Delta u_{t} . \\
Y^{T} y=-\int_{\mathcal{B}} u d m \equiv 0
\end{gathered}
$$

Onde, a última equação pode ser entendida como a definição de y.

Novamente, definindo a energia mecânica como na equação (3.36), obtemos que $E=T+V$. E, pelos mesmos cálculos, concluímos que ao longo das soluções,

$$
\dot{E}(t)=-2 \mathcal{D}\left(u_{t}\right) \text {. }
$$

Observe que neste caso, o anulamento da função $\mathcal{D}$ não é equivalente à ausência de deformação, como constatado nas seções (4.1) e (4.2).

O objetivo da dedução destas equações de movimento é o assunto apresentado na próxima seção.

\subsection{Tempo de relaxação}

Um tratamento para o sistema físico modelado na seção (4.4) foi extensivamente apresentado, principalmente por Michael Efroimsky, na série de artigos [LE99], [EL00], [Efr00], [Efr01] e [ELS02]. Pretende-se aqui, esboçar as ideias contidas principalmente na referência [Efr00]. Portanto, se alguma passagem matemática ou interpretação física ficarem obscuras, sugere-se ao leitor consultar este artigo. A abordagem proposta exige uma grande manipulação de expressões que, em sua maioria necessitam de argumentos físicos para serem asseguradas, transcendendo o método matemático. Por isso, os detalhes dos cálculos serão omitidos, exibindo-se somente o necessário para acompanhar 
as ideias. Foi considerado interessante exibi-lo nesta dissertação, com o fim de ilustrar a dificuldade na obtenção de previsões numéricas desses modelos.

Seja um corpo "aproximadamente rígido" como proposto na seção (4.4). Tome um referencial inercial com origem no centro de massa do mesmo. Em vista dos resultados (ou de princípios físicos), assumiremos que a energia cinética do corpo, $T_{\text {cin }}$, é decrescente, e que o momento angular (no referencial inercial) é conservado. Tome então um sistema de coordenadas (ortonormal) inercial no qual o momento angular $M$ está (fixo) ao longo do eixo $z$ (base $\left\{e_{x}, e_{y}, e_{z}\right\}$ ). No corpo, tome um referencial com origem no centro de massa e base (ortonormal), $\left\{e_{1}, e_{2}, e_{3}\right\}$, de autovetores do operador de inércia.

Consideremos as equações de Euler para o corpo rígido (2.2). Assumindo-se pequenas deformações, poderíamos pensar nos momentos de inércia $I_{1} \leq I_{2} \leq I_{3}$ dependentes do tempo. Portanto, seria válido questionar estas equações de movimento, propondo o sistema:

$$
\frac{d}{d t}\left(I_{i} \omega_{i}\right)=\left(I_{j}-I_{k}\right) \omega_{j} \omega_{k}
$$

Como este sistema é uma perturbação do original, as trajetórias da velocidade angular não devem se distanciar muito das originais. Assim, dadas as condições iniciais, pode-se pensar em período de rotação deste corpo, $\tau$.

Para uma rotação completa, no entanto, o autor afirma que $\dot{\omega}_{i} / \omega_{i} \approx \tau^{-1}$ e $\dot{I}_{i} / I_{i} \approx \epsilon \tau^{-1}$. Onde $\epsilon \approx 10^{-6}$ (usualmente), é um parâmetro dependendo das tensões relativas do material. Assim, é válido partir das equações usuais.

Procederemos agora com o chamado tratamento adiabático, o qual quantificaremos, mas que neste contexto é expresso pela suposição de que o decréscimo de energia é muito mais lento do que os movimentos de rotação e precessão do corpo, os quais são denominados processos rápidos.

Com isto, para uma rotação ou precessão completa, podemos assumir que a energia cinética, assim como o módulo do momento angular, são conservados (dados pela equação (2.3)).

Em vista da discussão na seção (2.2), espera-se novamente que "quase todo" movimento aproximese assintoticamente de uma rotação em torno do eixo de maior inércia (para caso não degenerado). Isto é equivalente a esperar que $\theta \stackrel{t \rightarrow \infty}{\longrightarrow} 0$. Onde $\theta$ é o ângulo entre os vetores momento e velocidade angular. Então é interessante estudar a evolução deste ângulo, ou melhor de sua média temporal no período de precessão.

Por motivos operacionais, sendo $T$ o período de precessão, é conveniente analisar

$$
\frac{d}{d t}\left\langle\operatorname{sen}^{2} \theta\right\rangle, \quad\left\langle\operatorname{sen}^{2} \theta\right\rangle:=\frac{1}{T} \int_{0}^{T} \operatorname{sen}^{2} \theta(s) d s .
$$

Como queremos trabalhar com condições iniciais próximas ao eixo de maior inércia, imaginamos que $d\left(<\operatorname{sen}^{2} \theta>\right) / d t<0$. Assim, sendo $\Omega$ a velocidade de precessão, o tratamento adiabático estará fundamentado na hipótese:

$$
-\frac{d}{d t}\left\langle\operatorname{sen}^{2} \theta\right\rangle \ll \Omega
$$

Assim, das conservações obtemos, com $P, Q, R, S$ constantes (dependentes somente dos momentos de inércia):

$$
\dot{\omega}_{2}^{2}=\left(\frac{I_{3}-I_{1}}{I_{2}}\right)^{2} \omega_{1}^{2} \omega_{3}^{2}=\left(\frac{I_{3}-I_{1}}{I_{2}}\right)^{2}\left(P-Q \omega_{2}^{2}\right)\left(R-S \omega_{2}^{2}\right) .
$$

Consultando a seção 14.1 da referência [SG59], constatamos que existem constantes $\beta, \Omega, k, \gamma, \alpha$, escritas em termos de $I_{1,2,3}, T_{\text {cin }}, M^{2}$, tais que a solução do sistema de equações é dada em termos 
das funções elípticas de Jacobi:

$$
\begin{aligned}
& \omega_{1}=\gamma \operatorname{cn}\left(\Omega\left(t-t_{0}\right), k^{2}\right) \\
& \omega_{2}=\beta \operatorname{sn}\left(\Omega\left(t-t_{0}\right), k^{2}\right) \\
& \omega_{3}=\alpha \operatorname{dn}\left(\Omega\left(t-t_{0}\right), k^{2}\right)
\end{aligned}
$$

Onde,

$$
\begin{gathered}
\alpha=\sqrt{\frac{M^{2}-2 I_{1} T_{\text {cin }}}{I_{3}\left(I_{3}-I_{1}\right)}}, \quad \beta=\sqrt{\frac{2 I_{3} T_{\text {cin }}-M^{2}}{I_{2}\left(I_{3}-I_{2}\right)}}, \quad \gamma=\sqrt{\frac{2 I_{3} T_{\text {cin }}-M^{2}}{I_{1}\left(I_{3}-I_{1}\right)}} \\
\Omega=\sqrt{\frac{\left(M^{2}-2 I_{1} T_{\text {cin }}\right)\left(I_{3}-I_{2}\right)}{I_{1} I_{2} I_{3}}}, \quad k=\sqrt{\frac{I_{2}-I_{1}}{I_{3}-I_{2}} \frac{2 I_{3} T_{\text {cin }}-M^{2}}{M^{2}-2 I_{1} T_{\text {cin }}}}
\end{gathered}
$$

Observação 4.5.1. A solução apresentada não está definida para quaisquer condiçôes iniciais. Lembrando que tratam-se das soluções do corpo rígido, constata-se que elas são válidas somente nas regiões, limitadas pelas separatrizes de sela, que contêm o eixo de maior inércia (veja figura (2.4)). Esta condição é equivalente a: $M^{2}>2 I_{2} T_{\text {cin }}$ (portanto, o denominador na definição de $k$ é não nulo).

Mas esta é a região de interesse neste estudo. Nas outras regiões, as soluções são parecidas.

Note que, perto das separatrizes de sela, não é razoável pensar em tratamento adiabático, pois espera-se que $\Omega \rightarrow \infty$ próximo a elas. Portanto, a análise apresentada é aplicável perto do polo $C$, interseção do eixo 3 com os elipsóides.

Dizemos que o corpo rígido é prolato se $I_{1} \leq I_{2}=I_{3}$, e oblato se $I_{1}=I_{2} \leq I_{3}$. Neste último caso, as soluçôes se reduzem às soluções clássicas de Euler: $\omega_{1}=\omega \cos \Omega\left(t-t_{0}\right), \omega_{2}=\omega \operatorname{sen} \Omega\left(t-t_{0}\right)$ $e \omega_{3}=$ constante.

O corpo está em rotação, logo, segundo o referencial fixo a ele, cada um dos pontos está sujeito à ação de uma "força fictícia", devida a sua aceleração. A resposta a esta é sua deformação, a qual está relacionada ao tensores de tensão e deformação, apresentados na seção (4.4). O próximo passo é estimar a dissipação de energia (que o autor denomina inelástica) associada a esta ação.

Denotando por $r, v, a$ e $r^{\prime}, v^{\prime}, a^{\prime}$ as posições, velocidades e acelerações dos pontos nos referenciais inercial e do corpo, respectivamente, das seções anteriores, sabemos:

$$
a=a^{\prime}+\dot{\omega} \times r^{\prime}+2 \omega \times v^{\prime}+\omega \times\left(\omega \times r^{\prime}\right) \approx \dot{\omega} \times r^{\prime}+\omega \times\left(\omega \times r^{\prime}\right) .
$$

Por argumentos análogos aos do início da seção, o autor argumenta que $a^{\prime}$ e $2 \omega \times v^{\prime}$ são desprezíveis em relação aos demais, portanto serão ignorados nos cálculos.

Procedendo como foi feito, podemos encontrar uma expressão para $\left\langle\operatorname{sen}^{2} \theta\right\rangle\left(T_{\text {cin }}\right)$. A partir de agora, começamos a "relaxar" o tratamento adiabático, considerando a variação de energia. Desta forma, fazemos:

$$
\frac{d}{d t}\left\langle\operatorname{sen}^{2} \theta\right\rangle=\frac{d}{d T_{\text {cin }}}\left\langle\operatorname{sen}^{2} \theta\right\rangle \frac{d T_{\text {cin }}}{d t} .
$$

Temos, $|M| \operatorname{sen} \theta=\left\|Y M \times e_{3}\right\|=\sqrt{I_{1}^{2} \omega_{1}^{2}+I_{2}^{2} \omega_{2}^{2}}=\sqrt{I_{1}^{2} R+\left(I_{2}^{2}-I_{1}^{2} S\right) \omega_{2}^{2}}$.

Portanto, o período de $\theta$ é igual ao período de $\omega_{2}^{2}$. Mas o período de $\omega_{2}=\beta \operatorname{sn}\left(\omega\left(t-t_{0}\right), k^{2}\right)$ é

$$
\tau=4 K\left(k^{2}\right):=4 \int_{0}^{\pi / 2}\left(1-k^{2} \operatorname{sen}^{2} \varphi\right)^{-\frac{1}{2}} d \varphi=2 \pi\left(1+\frac{k^{2}}{4}\right)+\mathcal{O}\left(k^{4}\right)
$$

Observação 4.5.2. Nas condiçôes estabelecidas, esperamos que $\omega_{1}, \omega_{2} \approx 0$. Portanto, das conservações, obtemos que $I_{3}^{2} \omega_{3}^{3} \approx 2 I_{3} T_{\text {cin }} \approx M^{2}$, ou seja, $2 I_{3} T_{\text {cin }}-M^{2} \approx 0$. Logo, $k \approx 0$, desde que o denominador que ocorre em sua definição não se aproxima de zero.

Em vista desta consideração, é interessante trabalhar com a expansão anterior.

Das propriedades destas funções de Jacobi, vemos que o período de $\theta$ é $\tau / 2=2 K\left(k^{2}\right)$. 
Portanto, $M^{2}\left\langle\operatorname{sen}^{2} \theta\right\rangle=I_{1}^{2} R+\left(I_{2}^{2}-I_{1}^{2} S\right) \beta^{2}\left\langle\operatorname{sn}^{2}\left(u, k^{2}\right)\right\rangle$.

$\mathrm{O}$ autor ainda afirma que

$$
\left\langle\operatorname{sn}^{2}\left(u, k^{2}\right)\right\rangle=\frac{1}{2 K} \int_{0}^{2 K} \operatorname{sn}^{2}\left(u, k^{2}\right) d u=\frac{1}{K} \int_{0}^{K} \operatorname{sn}^{2}\left(u, k^{2}\right) d u=\frac{1}{2}+\frac{1}{16} k^{2}+\frac{1}{32} k^{4}+\mathcal{O}\left(k^{6}\right) .
$$

Logo, usando também a definição de $\beta$, obtemos:

$M^{2} \frac{d}{d T_{\text {cin }}}\left\langle\operatorname{sen}^{2} \theta\right\rangle=\frac{I_{3}\left(I_{1} I_{3}+I_{2} I_{3}-2 I_{1} I_{2}\right)}{\left(I_{3}-I_{1}\right)\left(I_{3}-I_{2}\right)}+\frac{1}{4} \frac{\left(2 I_{3} T_{\text {cin }}-M^{2}\right)^{2}}{M^{2}-2 I_{1} T_{\text {cin }}}\left(\frac{I_{2}-I_{1}}{I_{3}-I_{2}}\right)^{2} \frac{I_{3}^{2}}{I_{3}-I_{1}}+\mathcal{O}\left(k^{4}\right)$.

Um caso particular interessante que se pode pensar é o oblato (desde que para o prolato, a região onde estes cálculos fazem sentido desaparece), fazendo $I_{2} \rightarrow I_{3}$. Obtendo:

$$
M^{2}\left(\frac{d}{d T_{\text {cin }}}\left\langle\operatorname{sen}^{2} \theta\right\rangle\right)^{\text {oblato }}=\frac{2 I_{1} I_{3}}{I_{3}-I_{1}} .
$$

Defina, $W$ como sendo a parcela da energia elástica armazenada no corpo dependente do tempo, devido a tensões "alternantes".

Pela definição, $\dot{T}_{c i n}=\dot{W}$, taxa de perda de energia elástica.

A deformação do corpo é constituída por um comportamento elástico e outro "plástico", este responsável pelo corpo não retornar exatamente à sua condição inicial após deformar-se.

Como sugerido na seção anterior, esta deformação é descrita pelos tensores de deformação e deformação viscosa. Definindo $\epsilon_{i j}:=\left(\partial_{i} u_{j}+\partial_{j} u_{i}\right) / 2$, é fácil constatar que:

$$
\begin{array}{ll}
\sigma_{i j}=(K \operatorname{Tr} \epsilon) \delta_{i j}+2 \mu\left(\epsilon_{i j}-\frac{1}{3} \delta_{i j} \operatorname{Tr} \epsilon\right), \quad \epsilon_{i j}=\frac{\operatorname{Tr} \sigma}{9 K} \delta_{i j}+\frac{1}{2 \mu}\left(\sigma_{i j}-\frac{1}{3} \delta_{i j} \operatorname{Tr} \sigma\right) \\
\tilde{\sigma}_{i j}=(\zeta \operatorname{Tr} \dot{\epsilon}) \delta_{i j}+2 \eta\left(\dot{\epsilon}_{i j}-\frac{1}{3} \delta_{i j} \operatorname{Tr} \dot{\epsilon}\right), \quad \dot{\epsilon}_{i j}=\frac{\operatorname{Tr} \tilde{\sigma}}{9 \zeta} \delta_{i j}+\frac{1}{2 \eta}\left(\tilde{\sigma}_{i j}-\frac{1}{3} \delta_{i j} \operatorname{Tr} \tilde{\sigma}\right) .
\end{array}
$$

Considere novamente o caso do corpo oblato, descrito na observação (4.5.1). A aceleração dada pela fórmula (4.22) fica assim na forma:

$$
\begin{gathered}
a=\left[-x\left(\omega_{3}^{2}+\omega^{2} / 2\right),-y\left(\omega_{3}^{2}+\omega^{2} / 2\right),-\omega^{2} z\right]+ \\
+\left[\omega_{3} \omega z \cos \Omega\left(t-t_{0}\right), \omega_{3} \omega z \operatorname{sen} \Omega\left(t-t_{0}\right), \omega_{3} \omega\left(x \cos \Omega\left(t-t_{0}\right)+y \operatorname{sen} \Omega\left(t-t_{0}\right)\right)\right]+ \\
+\omega^{2} / 2\left[y \operatorname{sen} 2 \Omega\left(t-t_{0}\right)+x \cos 2 \Omega\left(t-t_{0}\right), x \operatorname{sen} 2 \Omega\left(t-t_{0}\right)-y \cos 2 \Omega\left(t-t_{0}\right), 0\right] .
\end{gathered}
$$

Ou seja, a aceleração é composta por uma componente constante no tempo, outra oscilante de frequência $\Omega$ e outra de frequência $2 \Omega$. Vemos que a contribuição deste último termo não é desprezível. Este é um dos principais pontos levantados pelos autores nas cinco referências citadas. Estes entraram em desacordo com os resultados dos autores da referência [SBH05], que desprezaram esta contribuição em um artigo posterior.

A referência [SBH05] apresenta justificativas para tal procedimento, ressaltando as hipóteses que eles estavam fazendo sobre as condições de movimento e as propriedades do material constituinte. A principal aplicação que estão interessados é o movimento de asteróides e cometas.

Em vista desta evidência, propõe-se que a perda de energia é dada por uma superposição das energias dissipadas devido às oscilações em todos os modos normais $\left(\Omega_{n}\right)$ :

$$
\dot{W}=\sum_{\Omega_{n}} \dot{W}\left(\Omega_{n}\right)=-\sum_{\Omega_{n}} \frac{\Omega_{n} W_{0}\left(\Omega_{n}\right)}{Q\left(\Omega_{n}\right)}=-2 \sum_{\Omega_{n}} \frac{\Omega_{n}<W\left(\Omega_{n}\right)>}{Q\left(\Omega_{n}\right)} .
$$

Onde $Q\left(\Omega_{n}\right)$ é chamado de fator de qualidade do material constituinte do corpo (dado experimental), $W_{0}\left(\Omega_{n}\right)$ e $\left\langle W\left(\Omega_{n}\right)>\right.$, os valores máximo e médio (em um período) da energia elástica 
armazenada no corpo, correspondentes a vibrações de frequência $\Omega_{n}$.

O valor médio é dado por (fornecido pelo autor, sem mencionar referências)

$$
\langle W\rangle=\frac{1}{2} \int_{\mathcal{B}}\left\langle\sigma_{i j} \epsilon_{i j}\right\rangle d V+\frac{1}{2} \int_{\mathcal{B}}\left\langle\tilde{\sigma}_{i j} \tilde{\epsilon}_{i j}\right\rangle d V=\sum_{\Omega_{n}}\left\langle W\left(\Omega_{n}\right)\right\rangle .
$$

O qual, para temperaturas usuais, não possui contribuição da parte plástica dos tensores. Esta é uma abordagem estranha relativamente à seção anterior, onde a perda de energia estava ligada exatamente ao tensor com dependência nas velocidades.

Desta forma, o autor da referência propõe uma expressão mais geral para (4.24):

$$
\dot{W}=-2 \sum_{\Omega_{n}} \int_{\mathcal{B}} \frac{\Omega_{n}}{Q\left(\Omega_{n}\right)} \frac{d<W\left(\Omega_{n}\right)>}{d V} d V .
$$

Sugerindo que o fator de qualidade pode ter uma variação ao longo da extensão do corpo, porém deve ser muito pequena tendo em vista dados geológicos usuais (a discussão é restrita a asteróides e cometas).

Esta forma da equação foi proposta para estimar $d<W\left(\Omega_{n}\right)>/ d V$, a energia elástica associada ao modo $\Omega_{n}$ por unidade de volume (que será não desprezível somente para $\Omega_{1}=\Omega$ e $\Omega_{2}=2 \Omega$ ). Pois,

$$
\frac{d\langle W\rangle}{d V}=\frac{1}{2}\left\langle\sigma_{i j} \epsilon_{i j}\right\rangle=\sum_{\Omega_{n}} \frac{d<W\left(\Omega_{n}\right)>}{d V} .
$$

Logo, por um simples cálculo, obtém-se:

$$
\frac{d\langle W\rangle}{d V}=\frac{1}{4 \mu}\left(\left(\frac{2 \mu}{9 K}-\frac{1}{3}\right)\left\langle(\operatorname{Tr} \sigma)^{2}\right\rangle+\left\langle\sigma_{i j} \sigma_{i j}\right\rangle\right) .
$$

Observado os resultados da seção anterior, o tensor $\sigma$ deve ser tal que:

$$
\sigma_{i k} n_{k} \equiv 0, \quad \partial_{i} \sigma_{i j}=\rho a_{j} .
$$

Onde $n$ é o vetor normal ao bordo, $\rho$ é a densidade de massa e $a$ é dada por (4.22).

Observação 4.5.3. Note que a segunda condição é essencialmente uma simplificação da equação (4.19), considerando uma rotação rígida do corpo e desprezando os termos inerciais citados. Esta condição de contorno também foi utilizada na seção anterior.

Para facilitar a obtenção de $\sigma$ o autor faz a suposição de que o corpo é um prisma retangular de dimensões $2 \ell_{1}, 2 \ell_{2}, 2 \ell_{3}$. É argumentado que esta hipótese não compromete o resultado, com base no princípio de elasticidade de Saint-Venant. No entanto, se a geometria do corpo for "complicada" ou se possuir componentes de dimensão um ou dois, tal aproximação deixa de ser adequada.

A partir daqui, surge um extenso cálculo para obter a média das componentes do tensor $\sigma$. Estes serão omitidos, mas são encontrados no apêndice $\mathrm{C}$ de [Efr00]. O processo consiste em obter a aceleração $a$ a partir das expressões apresentadas em (4.21), por diferenciação. Em seguida, integrase a equação (4.27) para obter o tensor (observando a condição de contorno). Este processo não é direto, possui sutilezas relacionadas a aparecimento de "termos seculares" quando se aproxima as funções de Jacobi por funções trigonométricas da seguinte maneira:

$$
\begin{gathered}
\operatorname{cn}\left(u, k^{2}\right)=\cos u+\frac{1}{4} k^{2}(u-\operatorname{sen} u \cos u) \operatorname{sen} u+\mathcal{O}\left(k^{4}\right) \\
\operatorname{sn}\left(u, k^{2}\right)=\operatorname{sen} u-\frac{1}{4} k^{2}(u-\operatorname{sen} u \cos u) \cos u+\mathcal{O}\left(k^{4}\right) \\
\operatorname{dn}\left(u, k^{2}\right)=1-\frac{1}{2} k^{2} \operatorname{sen}^{2} u+\mathcal{O}\left(k^{4}\right)
\end{gathered}
$$

Calculando a média do tensor em um período de precessão, obtém-se $\dot{W}$, a partir de (4.26). 
Portanto, juntamente com (4.23), finalmente conclui-se que:

$$
\begin{aligned}
\frac{d}{d t}<\operatorname{sen}^{2} \theta & >=-\frac{2 I_{3} \rho^{2}}{\mu Q(\Omega)} \frac{2 I_{3} T_{\text {cin }}-M^{2}}{M^{2}}\left\{\Omega ( M ^ { 2 } - 2 I _ { 1 } T _ { \text { cin } } ) H _ { 1 } \left[\frac{I_{1} I_{3}+I_{2} I_{3}-2 I_{1} I_{2}}{\left(I_{3}-I_{1}\right)\left(I_{3}-I_{2}\right)}\right.\right. \\
& \left.+\frac{1}{4} \frac{I_{3}\left(I_{2}-I_{1}\right)^{2}}{\left(I_{3}-I_{1}\right)\left(I_{3}-I_{2}\right)^{2}} \frac{2 I_{3} T_{\text {cin }}-M^{2}}{M^{2}-2 I_{1} T_{\text {cin }}}\right]-\Omega H_{0}\left(2 I_{3} T_{\text {cin }}-M^{2}\right) \\
+ & \left.2 \Omega \frac{Q(\Omega)}{Q(2 \Omega)}\left(2 I_{3} T_{\text {cin }}-M^{2}\right) H_{2} \frac{I_{1} I_{3}+I_{2} I_{3}-2 I_{1} I_{2}}{\left(I_{3}-I_{1}\right)\left(I_{2}-I_{1}\right)}+\mathcal{O}\left(k^{4}\right)\right\} .
\end{aligned}
$$

Onde, as constantes $H_{0}, H_{1}, H_{2}$ dependem somente da geometria do corpo $\left(\ell_{1}, \ell_{2}, \ell_{3}\right)$, obtidas na integração da equação (4.27):

$$
\begin{gathered}
H_{0}=\frac{237}{m^{4}} \ell_{1} \ell_{2} \ell_{3}^{5}\left(\ell_{1}^{2}-\ell_{2}^{2}\right) \frac{\left(2.67 \ell_{1}^{4} \ell_{2}^{4}-\ell_{1}^{4} \ell_{3}^{4}-1.67 \ell_{2}^{4} \ell_{3}^{4}\right)\left(\ell_{1}^{4}+\ell_{2}^{4}-2 \ell_{3}^{4}\right)}{\left(\ell_{1}^{2}+\ell_{2}^{2}\right)\left(\ell_{1}^{2}-\ell_{3}^{2}\right)\left(\ell_{2}^{2}-\ell_{3}^{2}\right)\left(\ell_{1}^{4}-\ell_{3}^{4}\right)^{2}\left(\ell_{2}^{4}-\ell_{3}^{4}\right)^{2}} \\
H_{1}=\frac{317}{m^{4}} \frac{\ell_{1} \ell_{2} \ell_{3}^{5}}{\left(\ell_{2}^{2}+\ell_{3}^{2}\right)\left(\ell_{1}^{4}-\ell_{3}^{4}\right)\left(\ell_{1}^{2}+\ell_{2}^{2}\right)}\left(\frac{\ell_{2}^{4}}{\ell_{2}^{4}-\ell_{3}^{4}}+\frac{\ell_{1}^{4}}{\ell_{1}^{4}-\ell_{3}^{4}}\right) . \\
H_{2}=\frac{100}{m^{4}} \frac{\ell_{1}^{9} \ell_{2}^{9} \ell_{3}-\ell_{1}^{9} \ell_{2}^{5} \ell_{3}^{5}-\ell_{1}^{5} \ell_{2}^{9} \ell_{3}^{5}+0.21 \ell_{1}^{9} \ell_{2} \ell_{3}^{9}+0.19 \ell_{1}^{5} \ell_{2}^{5} \ell_{3}^{9}+0.21 \ell_{1} \ell_{2}^{9} \ell_{3}^{9}}{\left(\ell_{1}^{2}+\ell_{2}^{2}\right)^{2}\left(\ell_{1}^{4}-\ell_{3}^{4}\right)^{2}\left(\ell_{2}^{4}-\ell_{3}^{4}\right)^{2}}
\end{gathered}
$$

Mais interessante (e útil, segundo o autor, para observação de precessão de asteróides) do que a equação (4.28), é a equação que relaciona a velocidade de alinhamento, $d<\operatorname{sen}^{2} \theta>/ d t$ com $<\operatorname{sen}^{2} \theta>$. Nesta expressão, os termos constantes são constituídos pelos momentos de inércia, momento angular, fator de qualidade e densidade do material e as dimensões do corpo $\left(\ell_{1}, \ell_{2}, \ell_{3}\right)$. Esta é a equação (8.29) de [Efro0].

Afirma-se que parte destas quantidades pode ser medida com melhor precisão, o que permite inferir propriedades do corpo através desta fórmula. Nota-se que a equação é da forma (sendo $\left.\phi:=<\operatorname{sen}^{2} \theta>\right)$ :

$$
\frac{d \phi}{d t}=f(\phi)+\mathcal{O}\left(k^{4}\right) \approx f(\phi) .
$$

Podemos assim, a priori, estimar o tempo que $\phi$ gasta para evoluir de $\phi_{0}$ até $\delta \approx 0$ :

$$
t_{\text {relax }}:=\int_{\phi_{0}}^{\delta} \frac{d \phi}{f(\phi)}
$$

O qual é denominado tempo de relaxação do corpo.

Do ponto de vista observacional, este é um dado útil no caso de corpo oblato. Observa-se que para corpos com esta propriedade, é mais simples estimar $d \theta / d t$, cujo resultado exato é (referência [EL00]):

$$
\frac{d \theta}{d t}=-\frac{3}{16}\left(\frac{63\left(\ell_{3} / \ell_{1}\right)^{4} \cot ^{2} \theta+20}{\left(1+\left(\ell_{3} / \ell_{1}\right)^{2}\right)^{4}}\right) \frac{\ell_{1}^{2} M^{3} \rho}{\mu Q(\Omega)} .
$$

Há evidências numéricas de que o tempo de relaxação neste caso não é muito sensível à escolha de $\phi_{0}$. Portanto, considerando a precisão de um determinado aparelho, escolhe-se o valor de $\delta$ de forma que não seja possível distinguir uma rotação estacionária de uma outra com este ângulo de deflexão. Na ocasião de sua publicação, a referência [EL00] aponta o valor $\delta=6^{\circ}$ como compatível com os aparelhos de medida.

Assim, observando o tempo que um asteróide leva para cessar sua precessão, pode-se inferir quando se deu seu último impacto com outro corpo (de forma que sua rotação de equilíbrio fosse perturbada, não alterada drasticamente). No entanto, no artigo [EL00], os autores fazem uma estimativa deste tempo para o asteróide Toutatis 4179 , que possui um diâmetro de $4 \mathrm{~km}$ e um período de rotação de 7.5 dias. É tratado como um corpo oblato, obtendo-se:

$$
t_{\text {relax }} \lesssim 1.6 \times 10^{10} \text { anos }
$$


Ou seja, não é possível medir este tempo para este asteróide, mas pode-se usar a mesma ideia na observação da evolução do ângulo $\theta$. Em [EL00], é avaliada esta possibilidade.

O autor cita referências informando que no sistema solar a maioria dos asteróides e cometas estão no estado de rotação de equilíbrio, justificando de certa forma estes cálculos, e tornando viável a medida deste tempo, já que é frequente a ocorrência de corpos oblatos. 


\section{Conclusões}

O trabalho concentrou-se em uma pesquisa bibliográfica principalmente sobre o problema do corpo sólido munido de componentes elásticas.

Procurou-se dar uma visão própria sobre o assunto e os resultados das referências estudadas, sempre que possível explorando as analogias presentes.

Constatou-se que a conservação do momento angular para os problemas de contínuo fornecem condições de contorno que auxiliam a análise das soluções. No entanto, não se simplifica tanto o problema como no caso de mecânica de partículas, onde a redução na dimensão das coordenadas é, em boa parte dos exemplos, fundamental. Este aspecto foi notado nas seções (2.1) e (2.3). A dissipação de energia parece ser mais interessante nestes problemas, devido à forma que geralmente possui, que limita a norma da variável de deformação.

Como é conhecido do formalismo Hamiltoniano da mecânica clássica, no espaço de fase não podem ocorrer pontos de equilíbrio atratores (ou repulsores). Nos exemplos (em dimensão finita) apresentados aqui eles ocorreram no entanto. Observa-se que se o equilíbrio ocorre em mínimo local da energia mecânica (restrito à condição de momento angular constante), há um "sentido de estabilidade" associado a ele. O teorema (4.2.1), somente enunciado, estabelece uma certa analogia neste aspecto.

Seria interessante também tratar problemas de elasticidade usando uma abordagem alternativa à Lagrangiana.

\subsubsection{Propostas de estudo}

Na seção (2.3), o teorema (2.3.3) assegurou a existência de catorze pontos de equilíbrio sobre cada superfície de momento angular constante, porém não foi determinado o comportamento das soluções próximas a eles.

Uma das dificuldades encontradas nesta análise é que a superfície invariante, dada pela equação (2.7), tem dimensão 4.

O autor da referência estudada, [Lev89], fez uma ilustração de tal comportamento, porém não fez uma análise completa, afirmando que esta apareceria em um artigo posterior, o qual não foi encontrado. Pretendia-se tratar aqui este problema, mas não houve êxito.

Na seção (4.1), mostrou-se que $\dot{E}=-2 \mathcal{D}\left(u_{t}\right) \leq 0$, com $E \geq 0$. Esta é uma situação análoga à explorada no teorema (2.3.1).

Sugere-se supor que $u_{t}(t, \cdot)$ convirja uniformemente para uma função definida em $\mathcal{B}$. Assim, usando as mesmas ideias deste teorema, seria interessante mostrar que esta função tem que ser identicamente nula.

Finalmente, a principal proposta está relacionada aos resultados das seções (4.4) e (4.5). Na última, após um longo desenvolvimento, com aproximações e uso de dados experimentais, chegouse a uma estimativa do tempo de relaxação (equação (4.29)).

Fazendo uma observação sobre este procedimento, não ficou claro o uso da expressão (4.25) para a energia de deformação média armazenada no corpo, de forma a ser compatível com o desenvolvimento da seção (4.4).

De fato, após um simples cálculo, constata-se que a energia potencial dada em (4.14) pode ser 
escrita como:

$$
V=\int_{\mathcal{B}} \mu\left(\epsilon_{i k}-\frac{1}{3} \delta_{i k} \operatorname{Tr} \epsilon\right)^{2}+\frac{K}{2}(\operatorname{Tr} \epsilon)^{2} d V=\frac{1}{2} \int_{\mathcal{B}} \epsilon_{i k} \sigma_{i k} d V .
$$

E, analogamente,

$$
\mathcal{D}=\frac{1}{2} \int_{\mathcal{B}} \tilde{\epsilon}_{i k} \tilde{\sigma}_{i k} d V
$$

Logo a expressão proposta é: $\langle W\rangle:=V+\mathcal{D} \approx V$, para temperaturas não muito altas.

Mas, de acordo com a seção (4.4), $\dot{T}=\dot{E}-\dot{V}=-\dot{V}-2 \mathcal{D}$ a partir da qual não é claro que se pode desprezar a função dissipação, desde que sequer sabemos que $V$ é crescente próximo ao polo C (o que implicaria $\dot{T} \leq 0$ ). E ainda, isto faz com que no resultado final não ocorram as constantes de dissipação $\eta, \zeta$, surgindo o fator de qualidade.

Pensando de forma alternativa, o problema já estaria estabelecido, a priori, pelas equações (4.18), (4.19) e (4.20). Propõe-se assim obter uma estimativa para o tempo de relaxação a partir das mesmas. Desta maneira talvez fosse possível encontrar uma expressão para o fator de qualidade em termos das constantes de dissipação.

Não foi encontrado algum artigo onde tentou-se ou propou-se lidar com o problema desta forma, não aproximada, talvez pela dificuldade que ele possa impor. 


\section{Apêndice A}

\section{Princípios de Galileu}

O conteúdo deste apêndice é principalmente baseado na seção 2 do capítulo 1 da referência [Arn89].

Considere um sistema isolado de $N$ partículas, satisfazendo as leis de Newton:

$$
m_{i} \ddot{x}_{i}=f_{i}\left(t, x_{1}, \ldots, x_{N}, \dot{x}_{1}, \ldots, \dot{x}_{N}\right), \quad i=1, \ldots N, \quad x_{i} \in \mathbb{R}^{3}
$$

Os princípios de Galileu estabelecem que este sistema de equações deve ser invariante pelo grupo de transformações gerado pelas três seguintes famílias:

(i) $\mathcal{G}_{1}\left(t, x_{1}, \ldots, x_{N}, \dot{x}_{1}, \ldots, \dot{x}_{N}\right):=\left(t, x_{1}+t v, \ldots, x_{N}+t v, \dot{x}_{1}+v, \ldots, \dot{x}_{N}+v\right), \quad v \in \mathbb{R}^{3}$.

(ii) $\mathcal{G}_{2}\left(t, x_{1}, \ldots, x_{N}, \dot{x}_{1}, \ldots, \dot{x}_{N}\right):=\left(t-t_{0}, x_{1}-x_{0}, \ldots, x_{N}-x_{0}, \dot{x}_{1}, \ldots, \dot{x}_{N}\right), \quad t_{0} \in \mathbb{R}, \quad x_{0} \in \mathbb{R}^{3}$.

(iii) $\mathcal{G}_{3}\left(t, x_{1}, \ldots, x_{N}, \dot{x}_{1}, \ldots, \dot{x}_{N}\right):=\left(t, G x_{1}, \ldots, G x_{N}, G \dot{x}_{1}, \ldots, G \dot{x}_{N}\right), \quad G \in O(3)$.

A partir deste princípios, pode-se estudar quais são as restrições que eles impõem às forças $f_{i}: \mathbb{R}^{6 N} \times \mathbb{R} \longrightarrow \mathbb{R}^{3}$.

Seja o campo $F: \mathbb{R} \times \mathbb{R}^{6 N} \longrightarrow \mathbb{R} \times \mathbb{R}^{6 N}, F(t, X, \dot{X}):=\left(1, \dot{x}_{1}, \ldots, \dot{x}_{N}, f_{1}(t, X, \dot{X}), \ldots, f_{N}(t, X, \dot{X})\right)$, onde $X:=\left(x_{1}, \ldots, x_{N}\right)$.

Afirmar que o sistema de equações deve ser invariante por estas transformações significa que, se $\varphi_{s}$ é o fluxo de $F$, então $\varphi_{s}=\mathcal{G}_{k}^{-1} \circ \varphi_{s} \circ \mathcal{G}_{k}, \quad k=1,2,3$ (supondo que o campo seja completo).

Mas disso decorre que:

$$
\begin{gathered}
\frac{\partial}{\partial s} \varphi_{s} \circ \mathcal{G}_{k}(t, X, \dot{X})=\frac{\partial}{\partial s} \mathcal{G}_{k} \circ \varphi_{s}(t, X, \dot{X}) . \\
\Rightarrow F\left(\varphi_{s} \circ \mathcal{G}_{k}(t, X, \dot{X})\right)=D \mathcal{G}_{k}\left(\varphi_{s}(t, X, \dot{X})\right) \frac{\partial}{\partial s} \varphi_{s}(t, X, \dot{X})=D \mathcal{G}_{k}\left(\varphi_{s}(t, X, \dot{X})\right) F\left(\varphi_{s}(t, X, \dot{X})\right) . \\
\Rightarrow F\left(\mathcal{G}_{k}(t, X, \dot{X})\right)=F\left(\varphi_{s} \circ \mathcal{G}_{k} \circ \varphi_{s}^{-1}(t, X, \dot{X})\right)=D \mathcal{G}_{k}(t, X, \dot{X}) F(t, X, \dot{X}) .
\end{gathered}
$$

Ainda,

$$
D \mathcal{G}_{1}=\left(\begin{array}{cccccccc}
1 & 0 & 0 & \cdots & 0 & 0 & \cdots & 0 \\
v^{T} & I d_{3} & 0 & \cdots & 0 & 0 & \cdots & 0 \\
v^{T} & 0 & I d_{3} & \cdots & 0 & 0 & \cdots & 0 \\
\vdots & \vdots & \vdots & \ddots & \vdots & \vdots & \ddots & \vdots \\
v^{T} & 0 & 0 & \cdots & I d_{3} & 0 & \cdots & 0 \\
0 & 0 & 0 & \cdots & 0 & I d_{3} & \cdots & 0 \\
\vdots & \vdots & \vdots & \ddots & \vdots & \vdots & \ddots & \vdots \\
0 & 0 & 0 & \cdots & 0 & 0 & \cdots & I d_{3}
\end{array}\right)
$$

É imediato constatar que $D \mathcal{G}_{2}=I d_{6 N+1}$ e que $D \mathcal{G}_{3}=\mathcal{G}_{3}$, desde que é linear.

Portanto, estas condições implicam que as forças $f_{i}$ devem satisfazer: 
(i) $f_{i}\left(t, x_{1}, \ldots, x_{N}, \dot{x}_{1}, \ldots, \dot{x}_{N}\right)=f_{i}\left(t, x_{1}+t v, \ldots, x_{N}+t v, \dot{x}_{1}+v, \ldots, \dot{x}_{N}+v\right), \quad v \in \mathbb{R}^{3}$.

(ii) $f_{i}\left(t, x_{1}, \ldots, x_{N}, \dot{x}_{1}, \ldots, \dot{x}_{N}\right)=f_{i}\left(t-t_{0}, x_{1}-x_{0}, \ldots, x_{N}-x_{0}, \dot{x}_{1}, \ldots, \dot{x}_{N}\right), \quad t_{0} \in \mathbb{R}, \quad x_{0} \in \mathbb{R}^{3}$.

(iii) $G f_{i}\left(t, x_{1}, \ldots, x_{N}, \dot{x}_{1}, \ldots, \dot{x}_{N}\right)=f_{i}\left(t, G x_{1}, \ldots, G x_{N}, G \dot{x}_{1}, \ldots, G \dot{x}_{N}\right), \quad G \in O(3)$.

Notando que se pode tomar $x_{0}=0$ em $(i i)$, conclui-se que $f_{i}$ independe da variável $t$, para $i=1, \ldots, N$. E assim, tomando $x_{0}=x_{j}$ em $(i i)$ e $t=0, v=-\dot{x}_{j}$ em $(i)$, conclui-se que cada $f_{i}$ depende somente das posições e velocidades relativas entre as partículas, e não relativas ao referencial.

Ou seja, $f_{i}=f_{i}\left(x_{1}-x_{i}, \ldots, x_{N}-x_{i}, \dot{x}_{1}-\dot{x}_{i}, \ldots, \dot{x}_{N}-\dot{x}_{i}\right)$, e

$$
\begin{gathered}
G f_{i}\left(x_{1}-x_{i}, \ldots, x_{N}-x_{i}, \dot{x}_{1}-\dot{x}_{i}, \ldots, \dot{x}_{N}-\dot{x}_{i}\right)= \\
=f_{i}\left(G\left(x_{1}-x_{i}\right), \ldots, G\left(x_{N}-x_{i}\right), G\left(\dot{x}_{1}-\dot{x}_{i}\right), \ldots, G\left(\dot{x}_{N}-\dot{x}_{i}\right)\right), \forall G \in O(3), i=1, \ldots, N .
\end{gathered}
$$

Em particular, serão compatíveis com os princípios de Galileu quaisquer $f_{i}$ da forma:

$$
f_{i}=\sum_{j=1}^{N} \phi_{i j}\left(\left\|x_{j}-x_{i}\right\|,\left\|\dot{x}_{j}-\dot{x}_{i}\right\|,<x_{j}-x_{i}, \dot{x}_{j}-\dot{x}_{i}>\right)\left(x_{j}-x_{i}\right) .
$$

Portanto, são compatíveis com tais princípios as forças gravitacionais (com ou sem dissipação):

$$
\left.f_{i}=\sum_{j=1}^{N}\left[\frac{m_{i} m_{j}}{\left\|x_{j}-x_{i}\right\|^{3}}+m_{i} \nu_{i} \nu_{j} \frac{<x_{j}-x_{i}, \dot{x}_{j}-\dot{x}_{i}>}{\left\|x_{j}-x_{i}\right\|^{2}}\right)\right]\left(x_{j}-x_{i}\right) .
$$

Desde que, restringindo este caso para $N=2$, tomando o referencial no centro de massa e chamando de $x$ a posição relativa entre as partículas, é obtida a equação (2.1). Isto garante que esta força satisfaz os princípios de Galileu. 


\section{Apêndice B}

\section{Equações de movimento para problema restrito}

O objetivo desta seção é obter a equação de movimento (4.14) a partir dos resultados apresentados na seção (3.2).

Note que, em vista dos vínculos impostos, a matriz de rotação (4.8) pertence a $S O(2)$ (ou a um mergulho de $S O(2)$ em $G L(3)$, que é um subgrupo de Lie). Portanto para obter as equações de movimento, basta calcular a projeção ortogonal de $M(3)$ sobre $T_{I d} S O(2)$.

Seja $\gamma:(-\varepsilon, \varepsilon) \longrightarrow S O(2)$ uma curva de classe $C^{\infty}$ tal que $\gamma(0)=I d$. Considerando novamente a equação (4.8), vemos que $\gamma$ somente pode ser da forma:

$$
\gamma(t)=\left(\begin{array}{ccc}
\cos \theta(t) & -\operatorname{sen} \theta(t) & 0 \\
\operatorname{sen} \theta(t) & \cos \theta(t) & 0 \\
0 & 0 & 1
\end{array}\right)
$$

Onde $\theta(0)=0$. Além disso, como $\gamma$ é suave e as funções seno e cosseno são difeos locais (sobre domínios diferentes), decorre que $\theta(t)$ também é suave. Logo,

$$
\dot{\gamma}(0)=\dot{\theta}(0)\left(\begin{array}{ccc}
0 & -1 & 0 \\
1 & 0 & 0 \\
0 & 0 & 0
\end{array}\right) \in T_{I d} S O(2) .
$$

Logo, $T_{I d} S O(2) \subset M(3)$ é o conjunto das matrizes da forma:

$$
A=\left(\begin{array}{ccc}
X & 0 \\
0 & 0 & 0
\end{array}\right)=\lambda\left(\begin{array}{ccc}
0 & -1 & 0 \\
1 & 0 & 0 \\
0 & 0 & 0
\end{array}\right) .
$$

Ou seja, tal que $X \in A n t(2) \subset M(2)$. Observe que, de fato, este é um espaço vetorial.

Para calcular $T_{I d} S O(2)^{\perp}$, tome $B \in M(3)$ :

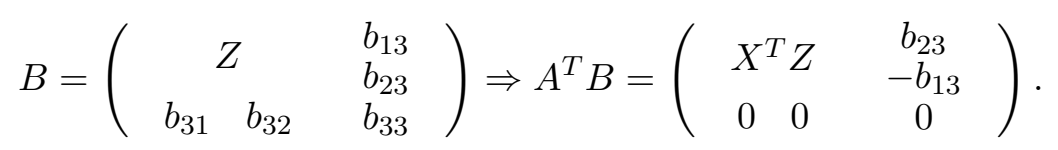

Logo, $\operatorname{Tr} A^{T} B=0 \Leftrightarrow \operatorname{Tr} X^{T} Z=0 \Leftrightarrow Z \in \operatorname{Sym}(2)$. Ou seja, $B \in T_{I d} S O(2)^{\perp}$ se e só se:

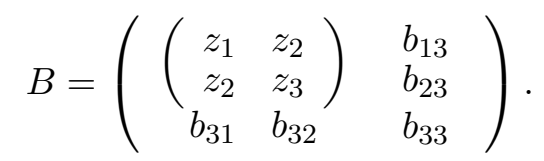


Assim, dados $Y \in S O(2)$ e $C \in T_{Y} G L(3)=Y M(3)=Y T_{I d} S O(2) \oplus Y T_{I d} S O(2)^{\perp}$, temos que

$$
C=Y\left(\begin{array}{ccc}
X & x_{13} \\
x_{31} & x_{32} & x_{23} \\
x_{33}
\end{array}\right)=Y\left(\begin{array}{ccc}
X_{a} & 0 \\
0 & 0 & 0
\end{array}\right)+Y\left(\begin{array}{ccc}
X_{s} & x_{13} \\
x_{31} & x_{32} & x_{33}
\end{array}\right) .
$$

Onde $X_{s}$ e $X_{a}$ são as partes simétrica e antissimétrica de $X$, respectivamente.

Denote por $\tilde{P}_{Y}$ a projeção ortogonal e $\pi: M(3) \longrightarrow M(2)$ a função definida por:

$$
\pi\left(\begin{array}{ccc}
Z & b_{13} \\
b_{31} & b_{32} & b_{23}
\end{array}\right):=Z
$$

Assim, da equação (B.1) decorre que:

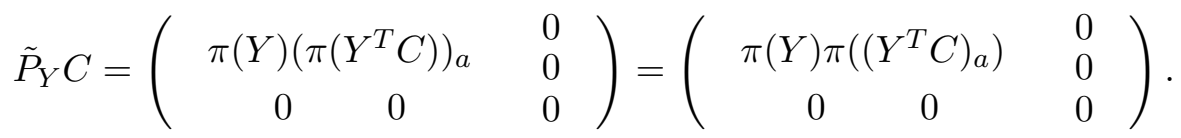

Faça

$$
C=\frac{d}{d t}\left(\frac{\partial T}{\partial \dot{Y}}\right)-\frac{\partial T}{\partial Y}
$$

Portanto, pela equação (3.11), vemos que

$$
Y^{T} C=\frac{d}{d t}\left(\frac{\partial K}{\partial \Omega}\right)+\left[\Omega, \frac{\partial K}{\partial \Omega}\right] \Rightarrow\left(Y^{T} C\right)_{a}=\dot{A}+[\Omega, A] .
$$

Observe que, devido à definição de $V$ e $\mathcal{D}$, o sistema em questão é livre de torque, logo a primeira equação de movimento fica:

$$
\tilde{P}_{Y}\left(\frac{d}{d t}\left(\frac{\partial T}{\partial \dot{Y}}\right)-\frac{\partial T}{\partial Y}\right)=0
$$

Logo, $\pi \circ S^{-1}(\dot{a}+\omega \times a)=2 \pi(\dot{A}+[\Omega, A])=0$.

Mas, pela definição do isomorfismo $S$ temos que:

$$
\left(\begin{array}{cc}
0 & -(\dot{a}+\omega \times a)_{3} \\
(\dot{a}+\omega \times a)_{3} & 0
\end{array}\right)=2 \pi(\dot{A}+[\Omega, A])=0 .
$$

Portanto, a primeira equação de movimento deste sistema consiste apenas na terceira componente da equação (4.4). Isto justifica a abordagem apresentada na seção (4.2).

A outra equação de movimento pode ser deduzida da maneira já apresentada. 


\section{Referências Bibliográficas}

[Arn89] V. Arnold. Mathematical Methods of Classical Mechanics. Springer, Berlin, segunda edição, 1989. 10, 21, 22, 59

[BL87] J. Baillieul e M. Levi. Rotational elastic dynamics. Physica D: Nonlinear Phenomena, 27:43-62, 1987. 3, 13, 21, 24, 39, 45

[BL91] J. Baillieul e M. Levi. Constrained relative motions in rotational mechanics. Archive For Rational Mechanics and Analysis, 115:101-135, 1991. 3, 24, 40

[BO78] C. M. Bender e S. A. Orszag. Advanced Mathematical Methods for Scientists and Engineers. McGraw-Hill, New York, primeira edição, 1978. 12

[Efr00] M. Efroimsky. Precession of a freely rotating rigid body. inelastic relaxation in the vicinity of poles. Journal of Mathematical Physics, 41:1854-1889, 2000. 3, 49, 53, 54

[Efr01] M. Efroimsky. Relaxation of wobbling asteroids and comets - theoretical problems, perspectives of experimental observation. Planetary and Space Science, 49:937-955, 2001. 3, 49

[EL00] M. Efroimsky e A. Lazarian. Inelastic dissipation in wobbling asteroids and comets. Monthly Notices of the Royal Astronomical Society, 311:269-278, 2000. 3, 49, 54, 55

[ELS02] M. Efroimsky, A. Lazarian e V. Sidorenko. Complex rotation with internal dissipation. applications to cosmic-dust alignment and to wobbling comets and asteroids. Recent Research Developments in Astrophysics, 2002. Research Signpost. 3, 49

[LB72] P. L. Lamy e J. A. Burns. Geometrical approach to torque free motion of a rigid body having internal energy dissipation. American Journal of Physics, 41:441-445, 1972. 10

[LE99] A. Lazarian e M. Efroimsky. Inelastic dissipation in a freely rotating body: application to cosmic dust alignment. Monthly Notices of the Royal Astronomical Society, 303:673-684, 1999. 3,49

[Lev89] M. Levi. Morse theory for a model space structure. Contemporary Mathematics, 97:209216, 1989. 14, 16, 17, 57

[LL86] L. D. Landau e E. M. Lifshitz. Theory of Elasticity. Pergamon Press, terceira edição, 1986. 48

[LL87] L. D. Landau e E. M. Lifshitz. Fluid Mechanics. Pergamon Press, segunda edição, 1987. 49

[Pon62] L. Pontryagin. Ordinary differential equations. Addison-Wesley, 1962. 8

[SBH05] I. Sharma, J. A. Burns e C.-Y. Hui. Nutational damping times in solids of revolution. Monthly Notices of the Royal Astronomical Society, 359:79-92, 2005. 3, 52

[SG59] L. Synge, J e B. A. Griffith. Principles of Mechanics. McGraw-Hill, New York, 1959. 50 
[Sot79] J. Sotomayor. Liçôes de Equações Diferenciais Ordinárias. Projeto Euclides, Rio de Janeiro, 1979. 16 